\title{
Correlación
}

\section{en los modelos de una y dos partículas en una caja.}

Tesis para obtener el grado de Maestría en Ciencias

\author{
(Química) \\ que presenta: \\ Humberto Laguna Galindo \\ Asesor: Dr. Robin P. Sagar \\ Universidad Autónoma Metropolitana \\ Unidad Iztapalapa \\ División de Ciencias Básicas e Ingeniería
}

November 5, 2011 
A los galeros, por ser guía y compañía en el trayecto.

A mis padres, por el esfuerzo de años, especialmente. A los necios que nos han dado el ejemplo, por no cejar en el empeño de revolucionar, encontrando y despejando los caminos.

A los casi 56 años... 
4

\section{Agradecimientos}

Agradezco a Conacyt la beca que permitió desarrollar este estudio de maestría. Agradezco al programa PIFI de la SEP por los fondos para asistir a congresos donde las etapas de este trabajo fueron presentadas.

A quienes sin proponérselo han contribuido para generar los ánimos de seguir en el camino.

En especial quiero agradecer a mi asesor, Robin Sagar, por la paciencia, por el tiempo y por las discusiones, las que eran científicas y las que no, ambas igual de provechosas. 


\section{Índice}

1 Introducción $\quad 9$

2 Antecedentes 15

2.1 Aspectos de Mecánica Cuántica. . . . . . . . . . . . . . . 15

2.2 La función de Wigner. . . . . . . . . . . . . . . . . . . . . 19

2.3 Aspectos de Teoría de la Información. . . . . . . . . . . . . . 21

2.3.1 La entropía diferencial de Shannon . . . . . . . . 22

2.4 Correlación posición-momento. . . . . . . . . . 25

2.4 .1 Medidas de correlación . . . . . . . . . . . . 26

3 Entropía de Shannon de la función de Wigner, $s_{w}$. 31

3.1 Definición . . . . . . . . . . . . . . . 31

3.2 Interpretación de $s_{w} \ldots \ldots \ldots \ldots \ldots$

3.2.1 ¿Por qué trabajar con la función de Wigner y no con alguna otra función de distribución conjunta? . . . . 34

3.2.2 ¿Pueden interpretarse de alguna manera las regiones negativas de la función de Wigner? . . . . . . . . . 35 
3.2.3 $i s_{w}$ puede mantener la interpretación de una medida de la localización de la distribución? . . . . . . . . 37

4 Una-partícula-en-una-caja $\quad 39$

4.1 Relaciones de incertidumbre. . . . . . . . . . . . . . . 40

4.2 Entropía de Shannon de la función de Wigner. . . . . . . . . 42

4.3 Correlación posición-momento. . . . . . . . . . . 50

5 Dos-partículas-en-una-caja $\quad 57$

5.1 Localización y correlación debidas a la indistinguibilidad como funciones de $n_{2} \ldots \ldots \ldots \ldots \ldots$

5.1 .1 Entropía de Shannon de las marginales. . . . . . . . . 59

5.1.2 Entropía de Shannon de la densidad de pares. . . . . 63

5.1 .3 Información mutua. . . . . . . . . . . . 69

5.2 Efectos del confinamiento sobre las entropías informacionales. 75

5.2.1 Entropía de Shannon de las marginales. . . . . . . . 76

5.2.2 Entropía de Shannon de la densidad de pares. . . . . 83

5.2 .3 Información mutua. . . . . . . . . . . . . . . . 91

5.3 Correlación posición-momento. . . . . . . . . . . . 94

5.3.1 Relación entre la correlación posición-momento y la proveniente de la indistinguibilidad. . . . . . . . . 95

5.3.2 Relación entre el entanglement cuántico y la indistinguibilidad. . . . . . . . . . . . 96

6 Resumen de los resultados $\quad 99$ 
8 Perspectivas 107

9 Apéndice A 109

9.1 El enlace químico. . . . . . . . . . . . . . . . . . . . 109

10 Apéndice B 115

10.1 Una-partícula-en-una-caja: Tablas. . . . . . . . . . . . 115

10.2 Dos-partículas-en una-caja: Tablas. . . . . . . . . . . 117 


\section{Introducción}

La correlación es fundamental para la comprensión del mundo físico.

Las leyes físicas y químicas se establecen sobre el hecho empírico de que existe correlación entre ciertas variables. La mayoría de las leyes utilizadas para la descripción del mundo físico y para el diseño de dispositivos que aprovechan sus propiedades y características, establecen la existencia de una correlación perfecta, de ahí proviene su dependencia funcional. Con la expresión matemática de una ley es posible predecir el valor de una cantidad física conociendo el valor de aquellas con las cuales se encuentra correlacionada.

Las leyes del movimiento de la mecánica clásica están construidas sobre la correlación entre la posición $(x)$ y el momento $(p)$ de los cuerpos. Si conocemos la posición inicial de una partícula, y permitimos que el sistema evolucione en el tiempo, podemos predecir la nueva posición a un tiempo $t^{\prime}$ sólo conociendo la velocidad. Existe entre estas dos variables una correlación perfecta.

En la mecánica cuántica hemos perdido la posibilidad de relacionar fun- 
cionalmente la posición y el momento de una partícula en virtud del principio de incertidumbre y es posible hablar, en este sentido, de correlación imperfecta entre $x$ y $p$. La dinámica del sistema no se describe más utilizando las leyes de Newton, que correlacionan ambas variables, sino mediante la ecuación de Schrödinger, que involucra solamente $x$ y $t$ ó $p$ y $t$.

Esta diferencia entre las descripciones clásica y cuántica de un sistema es de gran interés teórico, pues tiene que ver con la cuestión de cuándo escribir la ecuación clásica para describir un sistema y cuándo la ecuación cuántica, se trata de la transición mecánica clásica - mecánica cuántica.

En la mecánica cuántica existen tres tipos de correlación, una debida a las interacciones coulómbicas entre partículas cargadas, que aparece también en la mecánica clásica, otra proveniente de la indistinguibilidad de las partículas, por la estadística cuántica que el sistema debe obedecer, y la correlación posición-momento, que contiene una componente proveniente del álgebra no-conmutativa de los operadores correspondientes.

En general, la correlación ha sido analizada utilizando herramientas provenientes de la teoría estadística, como la covarianza, el coeficiente de correlación [1] y la información mutua [2, 3]. En la Química Cuántica la correlación se ha estudiado usando la energía de correlación, definida como la diferencia entre la energía calculada en el nivel de Hartree-Fock y la energía exacta.

Para estudiar la correlación posición-momento es deseable contar con una función que dependa en ambas variables, esto se consigue utilizando formulaciones de la mecánica cuántica en un espacio-fase [4, 5]. 
La correlación posición-momento puede pensarse en dos sentidos, complementarios. Por una parte si existe una correlación entre la posición y el momento de una partícula puede decirse que hay una cierta reminiscencia de la mecánica clásica, donde estas variables se encuentran perfectamente correlacionadas en la segunda ley de Newton, por ello es posible argumentar que podríamos recuperar una descripción clásica del sistema a través de la predicción de esta correlación posición-momento.

Por otro lado, la no-conmutatividad de los operadores de posición y momento es otro tipo de correlación, pues provoca que la determinación de la posición influya en la determinación del momento, y viceversa, esta noconmutatividad es una característica de la mecánica cuántica que provoca problemas de interpretación y está relacionada con fenómenos como el entanglement, de manera que esta componente de la correlación posiciónmomento bien puede denominarse "correlación cuántica".

En lo que refiere a la correlación coulómbica y la proveniente de la indistinguibilidad, en la descripción de algunos aspectos de un sistema cuántico es de mayor importancia incluir la segunda para reproducir sus características fundamentales, ejemplo de ello es que si uno incorpora la indistinguibilidad a un modelo no interactuante es posible recuperar de forma cualitativamente correcta algunas propiedades del sistema.

En este trabajo estudiaremos la correlación proveniente de la indistinguibilidad y la corrrelación posición-momento, discutiendo algunos aspectos específicos de la correlación cuántica. Es lícito preguntar cómo se relacionan la correlación debida a la indistinguibilidad y la que proviene de la 
no-conmutatividad.

La correlación debida a la simetría de la función de onda en el caso electrónico se manifiesta en el principio de exclusión de Pauli. La correlación debida a la no-conmutatividad de los operadores asociados a la posición y el momento de las partículas en el formalismo de la mecánica cuántica se manifiesta en el principio de incertidumbre de Heisenberg.

Los modelos de una partícula libre en una caja y de dos partículas libres en una caja permiten estudiar estos dos tipos de correlación.

Las herramientas utilizadas en esta tesis para estudiar la correlación provienen de la teoría de la información [6]. La entropía de Shannon puede interpretarse como una medida de la incertidumbre en una distribución probabilística, y por tanto como una medida de su deslocalización. La información mutua es una distancia estadística (no define una métrica) entre dos distribuciones probabilísticas, una de interés y otra que es el producto de sus marginales, y se interpreta como la información contenida en una variable acerca de la otra, es decir es una medida de la correlación. Para contrastar con la covarianza y el coeficiente de correlación, cabe mencionar que la información mutua es una medida más general de la correlación porque, a diferencia de las primeras, no sólo es sensible a la correlación lineal [7].

En términos cronológicos, este trabajo se inició con el estudio de unapartícula-en-una-caja, desde la perspectiva de las relaciones de incertidumbre, basado en estudios previos de Majerník, et. al., [8, 9] con relación a la energía. El análisis se extendió a estudiar el comportamiento de las en- 
tropías de Shannon con respecto de la longitud de la caja, luego se estudió el sistema de dos-partículas-en-una-caja [10] en ambos espacios, desde el punto de vista de la localización/deslocalización usando la entropía de Shannon de pares, y la de sus marginales, y también de la correlación entre variables debida a la indistinguibilidad, utilizando la información mutua, se estudió también el efecto del confinamiento sobre estas cantidades.

La meta era analizar cómo la simetría de la función de onda influye en la localización/deslocalización de las densidades de pares y de una partícula en los espacios de posición y momento y ver si la correlación inducida por la simetría en la función es diferente en ambos espacios.

Enseguida se inició el estudio del formalismo mecanocuántico en un espacio-fase, utilizando la función de quasiprobabilidad de Wigner [11], y se aplicó a estudiar la correlación posición-momento en el modelo de una-partícula-en-una-caja, usando la entropía de Shannon de la función de Wigner y la información mutua. Este análisis se extendió al modelo de dos-partículas-en-una-caja.

El objetivo era analizar la correlación posición-momento, y la relación que ésta tiene con la correlación que proviene de la simetría en la función de onda.

El orden lógico en que se presenta la tesis es como sigue: el capítulo 2 introduce las definiciones y los conceptos relevantes que han de utilizarse en el desarrollo; en el capítulo 3 se introduce la definición de la entropía de Shannon de la función de Wigner y su interpretación, el capítulo 4 discute el modelo de una-partícula-en-una-caja usando la entropía de Shannon, en 
relación con el principio de incertidumbre, y la correlación entre $x$ y $p$; el capítulo 5 discute el modelo de dos-partículas-en-una-caja, en relación con los tipos de correlación mencionados; en el capítulo 6 se ofrece un resumen puntual de los resultados obtenidos; en el capítulo 7 se pueden encontrar la discusión y las conclusiones generales de este estudio; finalmente, el capítulo 8 discute las perspectivas de esta línea de investigación; en el Apéndice A se detallan algunos resultados obtenidos sobre la molécula de $H_{2}$ y que tienen relación con las perspectivas a futuro de esta investigación; y en el Apéndice B se presentan tablas con los datos que se han graficado a lo largo del trabajo. 


\section{Antecedentes}

\subsection{Aspectos de Mecánica Cuántica.}

La descripción de los sistemas cuánticos se realiza por medio de la Ecuación de Schrödinger, cuyo caso unidimensional de una partícula se escribe,

$$
-\frac{\hbar}{i} \frac{\partial \psi(x, t)}{\partial t}=-\frac{\hbar^{2}}{2 m} \frac{\partial^{2} \Psi(x, t)}{\partial x^{2}}+V(x, t) \Psi(x, t) .
$$

Esta ecuación gobierna el comportamiento en el tiempo del sistema. Y dice que si podemos conocer el estado del sistema en el tiempo $t$, la aplicación consecuente de la ecuación de Schrödinger dependiente del tiempo permite conocer el estado en que se encontrará el sistema en el tiempo $t+\Delta t$.

Cuando el potencial sólo es función de la posición y no del tiempo, es posible separar las variables de la ecuación dependiente del tiempo, lo que implica que podemos escribir,

$$
\Psi(x, t)=\psi(x) f(t)
$$

donde $f(t)$ es la fase de la onda y usualmente tiene la forma $e^{-i t}$, cabe anotar 
que la definición de la fase es arbitraria y no tiene importancia en muchas de las aplicaciones de la teoría cuántica. En este caso, se dice que tratamos con un estado estacionario, para los que puede escribirse la ecuación de Schrödinger independiente del tiempo en el espacio de posición,

$$
-\frac{\hbar^{2}}{2 m} \frac{\partial^{2} \psi(x)}{\partial x^{2}}+V(x) \psi(x)=E \psi(x)
$$

donde $E$ es una constante de separabilidad y puede identificarse con la energía del sistema. Es posible escribir esta expresión en términos de las coordenadas de momento.

Usualmente, la descripción mecanocuántica de un sistema se hace bien en las coordenadas de la posición o del momento. Los estados del sistema están dados por una función de onda $[\psi(x)$ ó $\widetilde{\psi}(p)]$ que, se postula, contiene toda la información del estado del sistema y que está definida en un espacio de Hilbert. La función de onda depende en una sola variable, la posición o el momento. Ambas representaciones están relacionadas por la Transformada de Dirac-Fourier [12],

$$
\widetilde{\psi}(p)=\frac{1}{2 \pi} \int \psi(x) e^{\frac{-i p x}{\hbar}} d x
$$

Esta relación entre los espacios es consecuencia de que los operadores de posición y momento no conmutan, lo que también provoca que entre sus incertidumbres se establezca una relación, el llamado principio de incertidumbre de Heisenberg [13]. Existen diferentes maneras de expresar esta desigualdad, y de ello depende la cota que se impone sobre las incertidum- 
bres. El principio de incertidumbre suele escribirse como,

$$
\Delta x \Delta p \geq \frac{\hbar}{2}
$$

donde $\Delta x$ y $\Delta p$ son las desviaciones estándar de la posición y del momento, respectivamente. Los estados que cumplen con la cota inferior son llamados "estados de mínima incertidumbre" o coherentes, es el caso de las funciones gaussianas, soluciones del oscilador armónico.

Una forma diferente de escribir el principio de incertidumbre fue introducida por Schrödinger [14],

$$
(\Delta x)^{2}(\Delta p)^{2} \geq\left(\frac{<\hat{x} \hat{p}+\hat{p} \hat{x}>}{2}-<\hat{x}><\hat{p}>\right)^{2}+\left|\frac{<\hat{x} \hat{p}-\hat{p} \hat{x}>}{2}\right|^{2}
$$

El primer término del lado derecho de la desigualdad es la covarianza cuántica entre $\hat{x}$ y $\hat{p}, \operatorname{cov}(\hat{x}, \hat{p})$, elevada al cuadrado. El principio de incertidumbre puede relacionarse con la correlación estadística existente entre $\hat{x}$ y $\hat{p}$.

De manera análoga, Bialynicki-Birula y Mycielski [15] establecieron una relación de incertidumbre para estos observables en términos de las entropías de Shannon [6],

$$
s_{\psi}+s_{\widetilde{\psi}} \geq 1+\ln \pi
$$

donde,

$$
s_{\psi}=-\int d x|\psi(x)|^{2} \ln |\psi(x)|^{2}
$$




$$
s_{\widetilde{\psi}}=-\int d p|\widetilde{\psi}(p)|^{2} \ln |\widetilde{\psi}(p)|^{2}
$$

Una interpretación del principio de incertidumbre es que es imposible la determinación simultánea, con precisión arbitraria, de las dos observables. Dicho de otra manera, la determinación de $x$ con una cierta precisión (localización de la densidad) influye en la determinación de $p$ (deslocalización de la densidad) y viceversa, por lo que podemos decir que están correlacionados de alguna manera.

La suma entrópica puede expresarse como la entropía de una distribución separable en espacio-fase [16],

$$
s_{\psi}+s_{\widetilde{\psi}}=-\int d x d p|\psi(x)|^{2}|\widetilde{\psi}(p)|^{2} \ln \left[|\psi(x)|^{2}|\widetilde{\psi}(p)|^{2}\right]
$$

Un problema interesante es la definición de una entropía que utilice una forma más general de distribuciones de espacio-fase, como la función de Wigner.

Una característica importante del formalismo de la teoría cuántica es que según la interpretación de Copenhagen la función de onda carece de sentido físico (en el sentido de que no es susceptible de medirse) y es su norma al cuadrado lo que es posible interpretar como una densidad de probabilidad, con lo que se elimina la fase. 


\subsection{La función de Wigner.}

El principio de incertidumbre hace problemática la definición de una probabilidad conjunta de $x$ y $p$ que cumpla con las condiciones de la teoría de la probabilidad, y con ello hace problemática también la descripción del sistema mecanocuántico en un espacio-fase análogo al de la mecánica estadística clásica.

No obstante, en 1932 E. P. Wigner [11] utilizando como base la teoría de grupos, propuso una función que describe el sistema, dependiente en la posición y el momento y que utilizó para calcular correcciones cuánticas al equilibrio termodinámico. Con la función de Wigner pueden recuperarse todas las predicciones mecanocuánticas que provengan del formalismo convencional, pues es otra representación del operador de la densidad. Para una partícula que se mueve en una dimensión se define,

$$
W(x, p)=\frac{1}{\pi \hbar} \int d y \psi^{*}(x+y) e^{\frac{2 i p y}{\hbar}} \psi(x-y)
$$

y es claro que puede obtenerse a partir de la función de onda del sistema. Sin embargo, el formalismo que involucra la función de Wigner es independiente del formalismo de Schrödinger, pues ésta puede obtenerse también resolviendo la ecuación cuántica de Liouville.

La función de Wigner es una forma hermítica del vector de estado $\psi(x)$, por tanto debe ser real [18] y cumple con,

$$
\int d x d p W(x, p)=1
$$




$$
\begin{gathered}
\int d p W(x, p)=|\psi(x)|^{2}, \\
\int d x W(x, p)=|\widetilde{\psi}(p)|^{2}, \\
\left|\int d x \psi^{*}(x) \phi(x)\right|^{2}=(2 \pi \hbar) \int d x d p W_{\psi}(x, p) W_{\phi}(x, p) .
\end{gathered}
$$

Las propiedades descritas en las Ecs. (2.12), (2.13) y (2.14) y el hecho de ser una función real, son consistentes con las propiedades que se exigirían de una densidad de probabilidad conjunta para $x$ y $p$. Es relevante para la discusión en este trabajo que las marginales de la función de Wigner sean las densidades de posición y momento obtenidas a través del formalismo de Schrödinger. Existen otras funciones en espacio-fase, como la distribución $Q$ de Husimi, la distribución $P$ de Glauber-Sudarshan, y una familia de funciones parametrizadas [17], pero no poseen la propiedad de la distribución de Wigner de que sus marginales sean las densidades.

Por su parte, la Ec. (2.15) es el traslape entre dos funciones de Wigner asociadas a los estados $\psi(x)$ y $\phi(x)$, y tiene dos consecuencias interesantes, primero, si $\psi(x)=\phi(x)$,

$$
\int d x d p W^{2}(x, p)=\frac{1}{2 \pi \hbar}
$$

que excluye distribuciones muy localizadas del tipo $P_{\psi}(x, p)=\delta\left(x-x^{\prime}\right) \delta(p-$ $\left.p^{\prime}\right)[18], \delta$ es la función delta de Dirac. Segundo, si $\psi(x)$ y $\phi(x)$ son ortogonales, 


$$
\int d x d p W_{\psi}(x, p) W_{\phi}(x, p)=0
$$

que implica que la función de Wigner no es positivo-definida, de hecho sólo es positiva en todo el espacio para funciones gaussianas [18, 19], como el estado basal del oscilador armónico. Ambos casos son consecuencia de que la función de Wigner debe cumplir con el principio de incertidumbre.

Esta característica hace que se la llame, en general, una densidad de "cuasiprobabilidad", o una "cuasidensidad".

\subsection{Aspectos de Teoría de la Información.}

La Teoría de la Información es una disciplina probabilística que permite analizar propiedades de sistemas que involucran probabilidades o distribuciones de probabilidad y que surgió a partir de problemas en comunicación. La primera formulación se debe a Shannon [6]. La cantidad fundamental es la entropía de Shannon, que puede ser interpretada como una medida de la incertidumbre asociada al sistema, o como una medida de la localización si se trata en específico de una distribución de probabilidad.

Es por esto que una aplicación casi natural que encuentra la teoría de la información es en la mecánica cuántica, donde tratamos con densidades de probabilidad y donde conceptos como "incertidumbre" y "localización" son muy relevantes. Como consecuencia de esta unión ha surgido la Teoría de la Información Cuántica [20]. 
Al tratar sistemas cuánticos usualmente se utiliza la entropía de von Neumann, donde se utiliza la matriz de la densidad y está en función de sus eigenvalores. En este trabajo estamos interesados en tratar la incertidumbre asociada a la propia densidad en los espacios de posición, momento y en un espacio-fase cuántico, es decir, tratamos con variables continuas, por ello utilizaremos la formulación continua de la entropía de Shannon.

\subsubsection{La entropía diferencial de Shannon}

Si tenemos una distribución de probabilidad de la variable aleatoria $x, f(x)$, que cumple con $\int d x f(x)=1$. La entropía diferencial de Shannon (o simplemente entropía de Shannon) se define[6],

$$
s=-\int d x f(x) \ln f(x)
$$

Es posible definir la entropía de Shannon $\left(s_{N}\right)$ si la distribución está normalizada a un número, digamos $N$, cuya relación con la Ec. (2.18) es,

$$
s=\frac{s_{N}}{N}+\ln (N)
$$

cuando la densidad normaliza a $N$, la definición de la Ec. (2.18) es equivalente a utilizar en la entropía el factor de forma (shape function) [21] de dicha distribución.

Es posible definir una entropía relativa, que es una "distancia" estadística de una distribución de probabilidad respecto de una referencia, $q(x)$ [22], 


$$
s(f, q)=\int d x f(x) \ln \frac{f(x)}{q(x)} .
$$

Usando la propiedad de convexidad, se demuestra que $s(f, q) \geq 0$, con la igualdad sí y sólo sí $f(x)=q(x)$ [23].

Para casos bidimensionales, se define una entropía conjunta, dada una distribución de probabilidad conjunta que cumpla con todas las propiedades exigidas por la teoría de la probabilidad, digamos $f(x, p)$,

$$
s_{x, p}=-\iint d x d p f(x, p) \ln f(x, p) .
$$

Si definimos,

$$
s_{x}=-\int d x f_{1}(x) \ln f_{1}(x)
$$

y

$$
s_{p}=-\int d p f_{2}(p) \ln f_{2}(p)
$$

con,

$$
f_{1}(x)=\int d p f(x, p)
$$

y

$$
f_{2}(p)=\int d x f(x, p)
$$


puede verificarse que la entropía conjunta cumple con la propiedad de subaditividad,

$$
s_{x, p} \leq s_{x}+s_{p}
$$

y la igualdad se satisface sólo si los dos eventos son independientes.

Se puede definir también una entropía relativa para distribuciones de más de una variable, introduciendo una distribución de referencia, $q(x, p)$, como sigue,

$$
s(f, q)=\iint d x d p f(x, p) \ln \frac{f(x, p)}{q(x, p)} .
$$

Además, es posible definir una entropía relativa conjunta, cuya distribución de referencia es el producto de las marginales, esta es una medición de la correlación entre dos variables. Es la información mutua,

$$
I_{x, p}=\iint d x d p f(x, p) \ln \frac{f(x, p)}{f_{1}(x) f_{2}(p)}=s_{x}+s_{p}-s_{x, p}
$$

se desprende de la propiedad de subaditividad que la información mutua siempre es no negativa y sólo es cero cuando los eventos son independientes, $f(x, p)=f_{1}(x) f_{2}(p)$.

Otra medida de la correlación estadística entre variables es el coeficiente de correlación, cuyo numerador es la covarianza y se define,

$$
\tau=\frac{<x p>-<x><p>}{\sqrt{<x^{2}>-<x>^{2}} \sqrt{<p^{2}>-<p>^{2}}} .
$$




\subsection{Correlación posición-momento.}

Como se ha mencionado en la introducción, en la mecánica cuántica hemos perdido la posibilidad de medir la correlación posición-momento en una relación funcional.

¿Cómo definir entonces la correlación posición-momento?

Comencemos por clarificar el problema: una forma de la correlación posición-momento está expresada en el principio de incertidumbre, y consecuentemente en la cota impuesta sobre la suma entrópica, de donde se desprende que no es posible localizar arbitraria y simultáneamente ambas distribuciones.

Esta correlación proviene de la no conmutatividad de los operadores, y se manifiesta incluso en una distribución separable, pues hemos hecho notar más arriba que la suma entrópica puede verse como la entropía de una distribución de espacio-fase que es el producto de las densidades, Ec. (2.10).

Otra forma de la correlación posición-momento está relacionada con la posibilidad de inferir el valor de $p$ a partir del de $x$, y viceversa, para estudiarla es necesario tener una función que dependa en $x$ y $p$, por ejemplo la función de Wigner, $W(x, p)$. Esta forma de la correlación posición-momento se ha estudiado a partir de la función de Wigner utilizando al menos dos criterios.

El primero sugiere que si la función de Wigner es separable no puede predecirse correlación en el sistema, y por el contrario ésta se manifiesta 
en los picos de la función, pues si la función es suficientemente localizada, conociendo $x$ es posible inferir $p$ y viceversa. Este criterio es similar al de la covarianza (o su versión normalizada, el coeficiente de correlación) [5].

Un problema de esta medición de la correlación es que ésta puede predecirse si $W(x, p)$ es muy localizada, aunque fuese separable [4] . El concepto en el centro de esta predicción de la correlación es la localización de la distribución conjunta.

El segundo criterio utiliza una analogía entre un estado clásico y un estado coherente, planteando que el estado cuántico que "más clásicamente" se comporta es aquel en el que la incertidumbre en ambos espacios es la misma, esto estaría hablando de la máxima correlación entre $x$ y $p$ que es posible obtener en un sistema cuántico, pues son estados donde la medición de $x$ tiene el mismo efecto sobre $p$, que la medición de $p$ sobre $x$. La proyección de un estado arbitrario sobre un estado coherente sería en este sentido una medida de la correlación posición-momento [4].

\subsubsection{Medidas de correlación}

\section{Covarianza y funciones de correlación de orden mayor.}

Para estudiar la correlación se han utilizado cantidades definidas en la teoría estadística, e. g. la covarianza, como en [1].

En la teoría estadística, la covarianza clásica entre dos variables, digamos $x$ y $p$, se escribe, 


$$
C_{1,1}=\operatorname{cov}(x, p)=<x p>-<x><p>
$$

y si tratamos con potencias de las variables, conocidas como momentos más altos de la disribución, en general tenemos una función de correlación de orden mayor, definida,

$$
C_{n, m}=\operatorname{cov}\left(x^{n}, p^{m}\right)=<x^{n} p^{m}>-<x^{n}><p^{m}>
$$

Para formular una versión cuántica de la covarianza y de las funciones de correlación de orden mayor, sustituimos las variables $x$ y $p$ por los operadores $\hat{x}=x$ y $\hat{p}=-i \hbar \frac{d}{d x}$, pero tenemos el problema de que los operadores no conmutan, por lo que el cálculo de $<\hat{x}^{n} \hat{p}^{m}>$ no es directo, requiere de ordenar los operadores.

La covarianza cuántica para los operadores $\hat{x}$ y $\hat{p}$ se escribe,

$$
C_{1,1}=\operatorname{cov}(\hat{x}, \hat{p})=\frac{1}{2}<\hat{x} \hat{p}+\hat{p} \hat{x}>-<\hat{x}><\hat{p}>
$$

y en general, para momentos más altos de la distribución tenemos [24],

$$
C_{n, m}=\operatorname{cov}\left(\hat{x}^{n}, \hat{p}^{m}\right)=\frac{1}{2}<\hat{x}^{n} \hat{p}^{m}+\hat{p}^{m} \hat{x}^{n}>-<\hat{x}^{n}><\hat{p}^{m}>.
$$

En esta expresión, el anticonmutador, $\left[\hat{x}^{n}, \hat{p}^{m}\right]_{+}=\hat{x}^{n} \hat{p}^{m}+\hat{p}^{m} \hat{x}^{n}$, considera un cierto ordenamiento para calcular $\left\langle\hat{x}^{n} \hat{p}^{m}\right\rangle$. Sin embargo, en la literatura no hay acuerdo sobre cómo se debe calcular el valor esperado de este producto. Puede usarse en lugar del anticonmutador una versión 
totalmente simetrizada que tiene en cuenta todas las permutaciones. Para ilustrar este punto tomemos en cuenta el operador $\hat{x} \hat{p}^{2}$, que podemos escribir de una forma totalmente simetrizada como,

$$
\left\{\hat{x} \hat{p}^{2}\right\}_{\text {sim }}=\frac{1}{3}\left(\hat{x} \hat{p}^{2}+\hat{p}^{2} \hat{x}+\hat{p} \hat{x} \hat{p}\right) .
$$

Estas diferentes formas de ordenar conducen a diferentes valores esperados del producto. Se han propuesto pruebas experimentales para verificar alguna [25].

Resaltaremos que si tenemos en cuenta la forma totalmente simetrizada del producto de operadores, para calcular el valor esperado podemos utilizar la función de Wigner y escribir como si calculáramos el valor esperado clásicamente [17],

$$
<\left\{\hat{x}^{n} \hat{p}^{m}\right\}_{\operatorname{sim}}>=\int d x d p x^{n} p^{m} W(x, p) .
$$

Se sabe que la covarianza es sensible a la correlación lineal entre las variables.

\section{Información mutua.}

La teoría de la información provee otra herramienta para medir la correlación estadística entre variables, la información mutua, que definimos para $x$ y $p$ como,

$$
I=\int d x d p W(x, p) \ln \frac{W(x, p)}{\left.|\psi(x)|^{2}|| \widetilde{\psi}(p)\right|^{2}}=s_{\psi}+s_{\widetilde{\psi}}-s_{w}
$$


donde $s_{w}$ es la entropía de Shannon de la función de Wigner, cuya introducción es el tema del siguiente capítulo.

La información mutua es una medida más general de la correlación que la covarianza, pues es sensible además a la correlación no lineal entre las variables [7]. 


\section{Entropía de Shannon de la función de Wigner, $s_{w}$.}

\subsection{Definición}

De acuerdo con uno de los criterios para detectar la correlación posiciónmomento enunciados en el capítulo anterior, ésta se manifestaría en los picos de la función de Wigner [5], por lo que tener una medida de la estructura de esa distribución sería deseable.

Por otro lado, la información mutua es una medida de la correlación que depende en las entropías de Shannon de la distribución conjunta y de las marginales. Es un balance entre la localización manifestada en la distribución conjunta y la que se manifiesta en las marginales. Para calcularla utilizando la función de Wigner, requerimos también medir la localización.

En ambos casos en el centro del concepto de correlación se encuentra la localización de la distribución conjunta. Para medir esa localización la entropía de Shannon es una herramienta posible. 
Wehrl ha introducido el concepto de entropía de una distribución en espacio-fase [26] cuántico, y se ha definido la entropía de Wehrl utilizando la distribución de Husimi [27, 28].

Estamos interesados en utilizar la función de Wigner para definir una entropía de Shannon en un espacio-fase cuántico. En la literatura pueden encontrarse algunos casos en que se plantea el problema de calcular esta entropía, pero se evita calcularla debido a la presencia de regiones negativas $[29,30]$.

Definiremos la entropía de Shannon de la función de Wigner, $s_{w}$, que aparece en la Ec. (2.35) como,

$$
s_{w}=-\int d x d p W(x, p) \ln W(x, p)
$$

Como hemos anotado, la presencia de las regiones negativas ha provocado que en la literatura no se haya calculado, pero este no es un problema para la función logaritmo [31]. Para el cálculo de $s_{w}$ es deseable separar la función de Wigner. Escribimos primero para la normalización,

$$
N_{w}=\int_{+} d x d p W^{+}(x, p)+\int_{-} d x d p W^{-}(x, p)
$$

donde $\int_{+} \mathrm{y} \int_{-}$denotan que el espacio de integración es aquel donde la función de Wigner es positiva $(+)$ y negativa $(-)$, con la misma simbología para $W^{+}(x, p)$ y $W^{-}(x, p)$.

Ahora escribimos para la entropía, 


$$
\begin{aligned}
s_{w}= & -\int_{+} d x d p W^{+}(x, p) \ln W^{+}(x, p) \\
& -\int_{-} d x d p W^{-}(x, p) \ln W^{-}(x, p)
\end{aligned}
$$

y el segundo término puede factorizarse,

$$
\begin{array}{r}
-\int_{-} d x d p W^{-}(x, p) \ln W^{-}(x, p)=-\int_{-} d x d p W^{-}(x, p) \ln \left[(-1)\left|W^{-}(x, p)\right|\right] \\
=\left(-\int_{-} d x d p W^{-}(x, p) \ln \left|W^{-}(x, p)\right|\right)-\int_{-} d x d p W^{-}(x, p) \ln (-1) \\
=\left(-\int_{-} d x d p W^{-}(x, p) \ln \left|W^{-}(x, p)\right|\right)-i \pi \int_{-} d x d p W^{-}(x, p)
\end{array}
$$

donde las barras "| |" denotan el valor absoluto y hemos utilizado la convención de trabajar en la rama principal del logaritmo.

Podemos separar las partes real e imaginaria de $s_{w}$,

$$
R e\left[s_{w}\right]=-\int_{+} d x d p W^{+}(x, p) \ln W^{+}(x, p)-\int_{-} d x d p W^{-}(x, p) \ln \left|W^{-}(x, p)\right|
$$

e

$$
\operatorname{Im}\left[s_{w}\right]=-i \pi \int_{-} d x d p W^{-}(x, p) .
$$




\subsection{Interpretación de $s_{w}$.}

A partir de lo que hemos ido estableciendo en el transcurso de este trabajo, pueden formularse las siguientes preguntas:

\subsection{1 ¿Por qué trabajar con la función de Wigner y no con alguna otra función de distribución con- junta?}

Resulta relevante para medir la correlación entre $x$ y $p$ usando la información mutua que la función de Wigner sea la distribución que permite recuperar las distribuciones de $x$ y de $p$ que provienen de la función de onda, además, imponiendo algunas condiciones puede reconstruirse la función de onda a partir de ésta. Es también importante que la función de Wigner pueda reconstruirse de datos experimentales [32, 33]. Asimismo apoyan esta elección el que cumpla con la condición de normalización y el que sea una función real.

Existen diferentes argumentos que resaltan a la distribución de Wigner como la más adecuada, por ser más cercana a la idea que tenemos de una distribución conjunta [19], y por ser esta representación la que asocia a cada punto del espacio-fase un observable físico [34]. 


\subsection{2 ¿Pueden interpretarse de alguna manera las re- giones negativas de la función de Wigner?}

La primera cuestión en este sentido se podría plantear de esta manera: Si vamos a utilizar herramientas de la teoría de la probabilidad para analizar la distribución de Wigner, que es otra representación del operador de la densidad, ¿dicha distribución tendría que aceptar una interpretación probabilística?

$\mathrm{Al}$ respecto debe apuntarse que una probabilidad está definida de acuerdo con axiomas y que estos garantizan su positividad [35]. Por otro lado, la aparición en física de valores negativos donde deberían aparecer probabilidades ha motivado una discusión en torno a si a estos valores se les podría llamar "probabilidades" también, extendiendo el concepto [36, 37].

En una perspectiva histórica no existe ningún impedimento para pensar una distribución de cuasi-probabilidad como una "verdadera distribución de probabilidad", entendiendo esto último como una distribución susceptible de ser estudiada con las mismas herramientas que una distribución enteramente positiva.

Desde el punto de vista matemático, la teoría de la probabilidad se establece sobre la base de axiomas que debe cumplir una distribución probabilística [35] y que descansan a su vez en intuiciones que es posible formarse a través del contacto con el mundo real. De aquí deriva que las probabilidades $\left(p_{i}\right)$ deban ser cantidades reales, positivas, menores o iguales a uno, cuya suma sea justamente la unidad, estas probabilidades lo son en un sen- 
tido "frecuentista", es decir que se interpretan como la frecuencia relativa del evento al que le asociamos la probabilidad cuando el número total de eventos tiende a infinito. Para la formulación discreta de la entropía de Shannon, la condición $0 \leq p_{i} \leq 1$ implica que $s_{x} \geq 0$.

Para el caso continuo hemos perdido la condición $p(x) \leq 1$, pues $p(x)$ es una distribución de probabilidad cuya discretización $[p(x) \Delta x]$ ya no corresponde a la probabilidad de un evento puntual, sino a la probabilidad de que el evento ocurra en el intervalo $x+\Delta x$, sólo debe garantizarse que la integral sobre todo el espacio sea la unidad. Hemos removido una de las propiedades axiomáticas y la distribución no tiene una cota superior, lo cual implica que $s_{x}$ tampoco tiene ya una cota inferior. ¿Cuál sería entonces una razón matemática concluyente para que no podamos remover la cota inferior de $p(x)$ ?

El hecho de que las probabilidades se interpreten como "frecuencias relativas" hace patente que el concepto "probabilidad" tiene una fuerte carga experimental y puede argumentarse que también tiene una inconveniencia física, pues implica una cantidad infinita de experimentos realizados. Por otro lado, si las probabilidades se interpretan en un sentido bayesiano, es decir como "falta de información", es más factible pensar en que la cota inferior pueda ser removida [36].

Un argumento que se ha esgrimido es que con el fin de mantener la congruencia en el lenguaje probabilístico se les podría efectivamente llamar probabilidades, con la salvedad de que estas probabilidades negativas serían utilizadas como intermediarias en operaciones, tal como se hace con números 
negativos en operaciones que sólo admiten como resultado final un número natural [37].

Para abonar a esta argumentación, debemos decir que incluso se ha formulado la mecánica cuántica incluyendo las probabilidades extendidas $[38,39]$.

En otro sentido puede argumentarse que las regiones negativas provienen de la naturaleza cuántica del sistema, pues son una manifestación del principio de incertidumbre. Tales regiones negativas han sido relacionadas con fenómenos cuánticos como el entanglement [40, 41].

Pero no es posible formular el argumento en el sentido inverso, puesto que existen sistemas cuánticos que tienen asociada una función de Wigner positivo-definida, como es el caso de algunos estados del emblemático gato de Schrödinger, tan asociado con el entanglement cuántico.

En este sentido, es importante observar que la integral que aparece en la Ec. (3.6) es el volumen negativo de la función de Wigner. Entonces la parte imaginaria de $s_{w}$ es directamente proporcional al volumen de la parte negativa de la función de Wigner.

\subsection{3 $i s_{w}$ puede mantener la interpretación de una medida de la localización de la distribución?}

$\mathrm{Al}$ respecto, pueden explicitarse algunas de las consecuencias que la utilización de una distribución de "quasi-probabilidad" tendría sobre el empleo de las herramientas informacionales. 
Las regiones negativas provocan valores imaginarios en la entropía, si es posible interpretar tales regiones como causadas por la naturaleza cuántica del sistema (la no-conmutatividad de los operadores), puede argumentarse que también es posible interpretar los valores imaginarios en el mismo sentido. La covarianza cuántica de dos operadores que no conmutan contiene también una parte imaginaria [42].

La parte real de la entropía tiene contribuciones de ambas regiones de la función, positiva y negativa.

Para el caso de la información mutua, la propiedad de subaditividad, que implica que $I \geq 0$ se desprende de la propiedad de convexidad que depende a su vez de la positividad de la función de distribución de probabilidad. No puede establecerse por tanto una cota inferior para la información mutua si trabajamos con una función que puede asumir valores negativos.

En general, las aplicaciones de las herramientas informacionales a distribuciones con regiones negativas constituyen un problema abierto, y este trabajo es sólo una aproximación al mismo. 


\section{Una-partícula-en-una-caja}

El modelo de una-partícula-en-una-caja es uno de los pocos modelos solubles analíticamente en la mecánica cuántica y quizá el más sencillo, por ello se utiliza en los libros de texto para introducir e ilustrar algunos de los conceptos fundamentales de la teoría, tales como la cuantización de la energía por el efecto del confinamiento, la degeneración de los niveles energéticos, el principio de superposición de estados, el principio de incertidumbre, etcétera.

La aplicación de este modelo, sin embargo, no es exclusivamente ilustrativo, también ha sido utilizado como una primera aproximación a diferentes problemas, y su utilización tiene muchas ventajas didácticas. Sus aplicaciones son de lo más variado, se ha empleado por ejemplo para modelar transiciones electrónicas en el espectro visible de algunas moléculas [43], para estudiar el concepto de aromaticidad [44], para ilustrar procesos como la formación de enlaces químicos [45], para modelar aspectos relacionados con polímeros conjugados [46], y para describir el efecto del confinamiento en los llamados puntos cuánticos [47], entre otras. 
40

\subsection{Relaciones de incertidumbre.}

Para el sistema de una partícula libre sin espín en una caja unidimensional de longitud $a$, las soluciones de la ecuación de Schrödinger independiente del tiempo en el espacio de posición sólo son diferentes de cero dentro del intervalo $[0, a]$, y son bien conocidas,

$$
\psi(x)=\sqrt{\frac{2}{a}} \sin \left(\frac{n \pi x}{a}\right), \quad 0 \leq x \leq a .
$$

La Transformada de Dirac-Fourier de esta función genera los eigenestados en el espacio de momento [48],

$$
\begin{aligned}
& \widetilde{\psi}(p)=(-i) \sqrt{\frac{a}{\pi \hbar}} e^{-i a p / 2 \hbar}\left[e^{i n \pi / 2} \frac{\sin [(a p / \hbar-n \pi) / 2]}{(a p / \hbar-n \pi)}\right. \\
&\left.-e^{-i n \pi / 2} \frac{\sin [(a p / \hbar+n \pi) / 2]}{(a p / \hbar+n \pi)}\right], \quad-\infty \leq p \leq \infty .
\end{aligned}
$$

Peslak [49] ha determinado las expresiones para la desviación estándar en este sistema, como funciones de $a$ (el tamaño de la caja) y de $n$ (el estado cuántico del sistema),

$$
\Delta x=\frac{a}{2 \sqrt{3}} \sqrt{1-\frac{6}{n^{2} \pi^{2}}}
$$

y

$$
\Delta p=\frac{h n}{2 a} .
$$


Puede observarse que el producto $\Delta x \Delta p$ es independiente del tamaño de la caja,

$$
\Delta x \Delta p=\frac{h n}{4 \sqrt{3}} \sqrt{1-\frac{6}{n^{2} \pi^{2}}} .
$$

Para $n \rightarrow \infty, \Delta x \simeq \frac{a}{2 \sqrt{3}}$, mientras $\Delta p \simeq \infty$, lo que significa que en ese caso el momento está completamente indeterminado, independientemente de $a$, y por tanto no es posible extraer la afirmación central del principio de incertidumbre, esto es la imposibilidad de determinar simultáneamente con una precisión arbitraria dos observables canónicamente conjugados, como $\hat{x}$ y $\hat{p}$. Entonces en este caso el uso de la formulación del principio de incertidumbre en términos de las desviaciones estándar no es lo más adecuado.

Por otra parte, se ha reportado una expresión asintótica para la entropía de Shannon en el espacio de posiciones [50]:

$$
s_{\psi}=\ln (2 a)-1
$$

También se ha establecido un límite superior para $s_{\widetilde{\psi}}$ cuando $n \rightarrow \infty$ $[9]:$

$$
\lim _{n \rightarrow \infty}\left[s_{\widetilde{\psi}}\right]=-\ln (2 a)+\ln (8 \pi)+\frac{\varphi}{\pi}
$$

donde $\varphi=2 \pi(1-\gamma) \approx 2.6564$ y $\gamma$ es la constante de Euler.

Por lo que la suma $\left(s_{\psi}+s_{\widetilde{\psi}}\right)$ oscila entre $2.212(n=1)$ y $3.0697(n \rightarrow \infty)$ [9] y es independiente de $a$. 
La interpretación de que la suma es independiente de $a$, es que debido al principio de incertidumbre mientras aumentamos el tamaño de la caja deslocalizamos la distribución en el espacio de posición, pero provocamos una localización equivalente en la distribución en el espacio de momento. Este comportamiento es provocado por la existencia de una cota superior para la suma entrópica de este sistema.

\subsection{Entropía de Shannon de la función de Wigner.}

Para calcular la función de Wigner a partir de la Ec. (2.11) hay que tener cuidado al fijar los límites para la integración sobre $y$.

Hay que tener en cuenta que las funciones $\psi(x \pm y)$ sólo son diferentes de cero en el intervalo $[0, a]$; necesitamos satisfacer que $0 \leq x+y \leq a$ y $0 \leq x-y \leq a$. Esto conduce a los siguientes límites de integración sobre $y$ en la Ec. (2.11), [51]:

$$
\begin{array}{ll}
-x \leq y \leq+x, & \text { si } 0 \leq x \leq a / 2 \\
-(a-x) \leq y \leq+(a-x), & \text { si } a / 2 \leq x \leq a
\end{array}
$$

de donde se obtiene, para el intervalo $[0, a / 2]$,

$$
\begin{array}{r}
W(x, p)=\left(\frac{2}{\pi \hbar a}\right)\left\{\frac{\sin [2(p / \hbar-n \pi / a) x]}{4(p / \hbar-n \pi / a)}+\frac{\sin [2(p / \hbar+n \pi / a) x]}{4(p / \hbar+n \pi / a)}\right. \\
\left.-\cos \left[\frac{2 n \pi x}{a}\right] \frac{\sin [2 p x / \hbar]}{(2 p / \hbar)}\right\}
\end{array}
$$


mientras que para el intervalo $[a / 2, a]$, sólo se reemplaza $x$ por $a-x$, [51].

$W(x, p)$ contiene más de un término, lo que provoca que la integración para calcular $s_{w}$ deba hacerse numéricamente. Para el cálculo de $s_{w}$ se utilizó integración numérica en 2 dimensiones, utilizando una malla en espacio $x$ de 0.01 y en espacio $p$ de 0.1 y una cuadratura de Gauss-Legendre de 12 puntos para cada subintervalo, utilizando aritmética compleja con precisión doble, este esquema fue probado calculando las normalizaciones. A lo largo de este trabajo se utilizaron unidades atómicas.

En la figura (4.1) se muestra el comportamiento de la norma de la entropía de la función de Wigner y de la parte real con el tamaño de la caja, $a$, comparado con la suma de las entropías de Shannon en los espacios de posición y momento, para el estado basal. Todas las curvas se mantienen constantes, es relevante que este comportamiento ocurra para la entropía conjunta de $x$ y $p$, calculada usando la función de Wigner, y para la suma de entropías, porque puede inferirse que la información del principio de incertidumbre está contenida de la misma manera en ambas. Para $s_{\psi}+s_{\widetilde{\psi}}$ se ha reportado este comportamiento antes [9] y puede obtenerse analíticamente. Hasta donde sabemos, no se ha calculado para la función de Wigner.

Las figuras (4.1), (4.2), (4.3) y (4.4) muestran que el comportamiento de la suma entrópica es similar al comportamiento de la entropía de Shannon de la función de Wigner para diferentes estados, ambas son constantes con $a$.

La suma entrópica es un caso particular de la entropía de Shannon de una distribución en un espacio-fase cuántico, véase Ec. (2.10), una gener- 


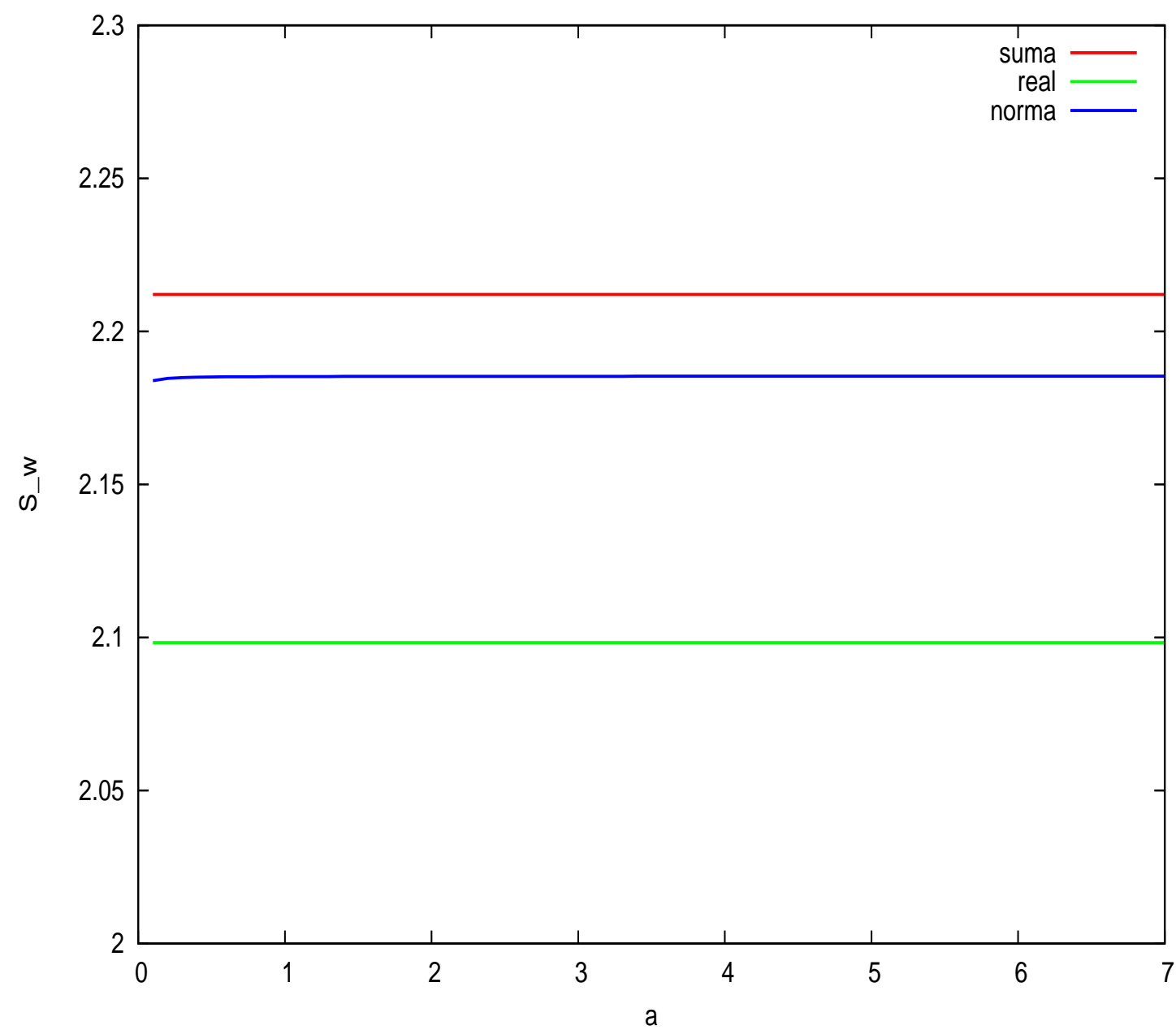

Figura 4.1: Parte real (verde) y norma (azul) de la entropía de Shannon de la función de Wigner, suma entrópica, $s_{\psi}+s_{\widetilde{\psi}}$, (rojo). Comportamiento con $a$ para $n=1$ 


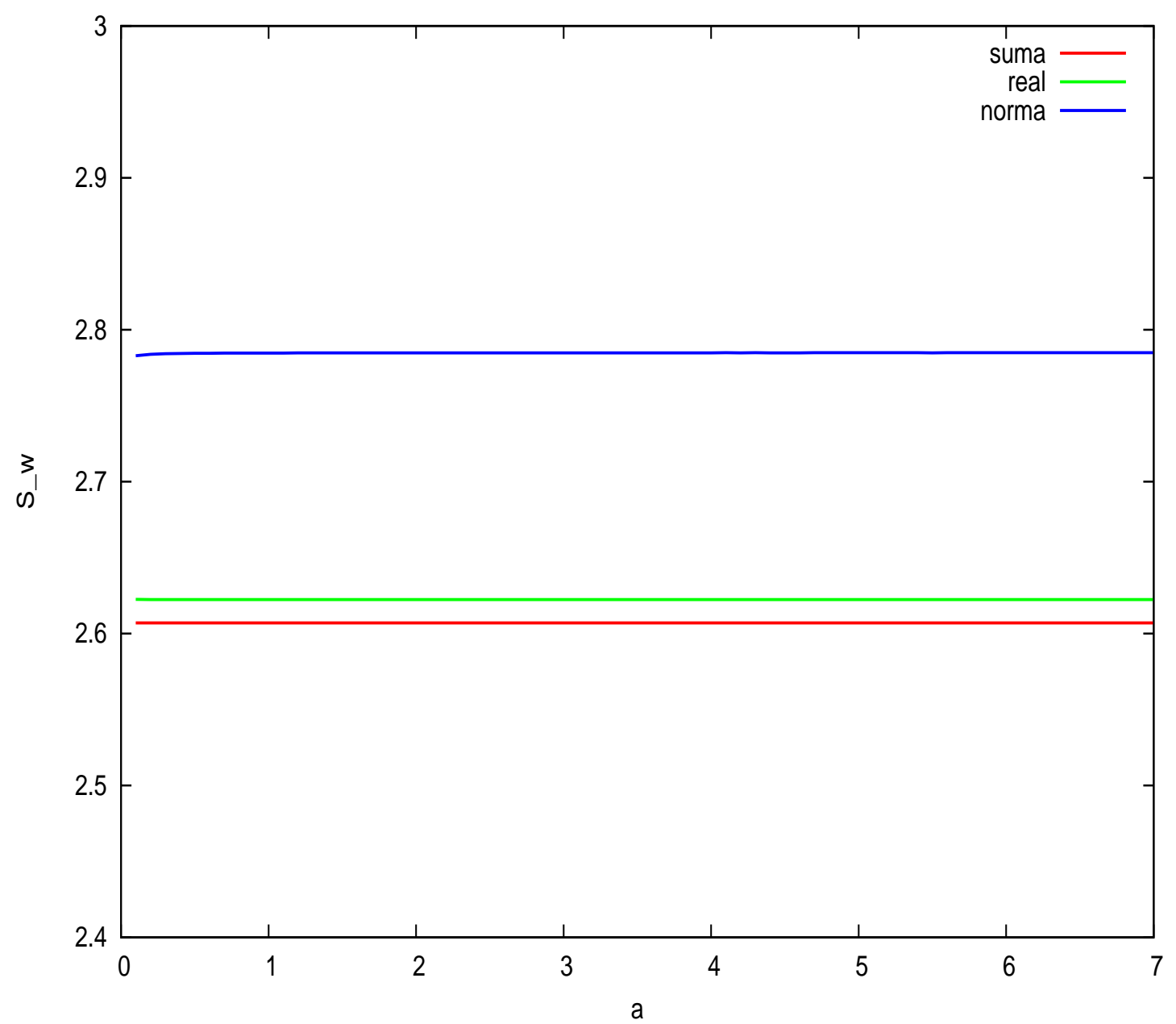

Figura 4.2: Parte real (verde) y norma (azul) de la entropía de Shannon de la función de Wigner, suma entrópica, $s_{\psi}+s_{\widetilde{\psi}}$, (rojo). Comportamiento con $a$ para $n=2$ 


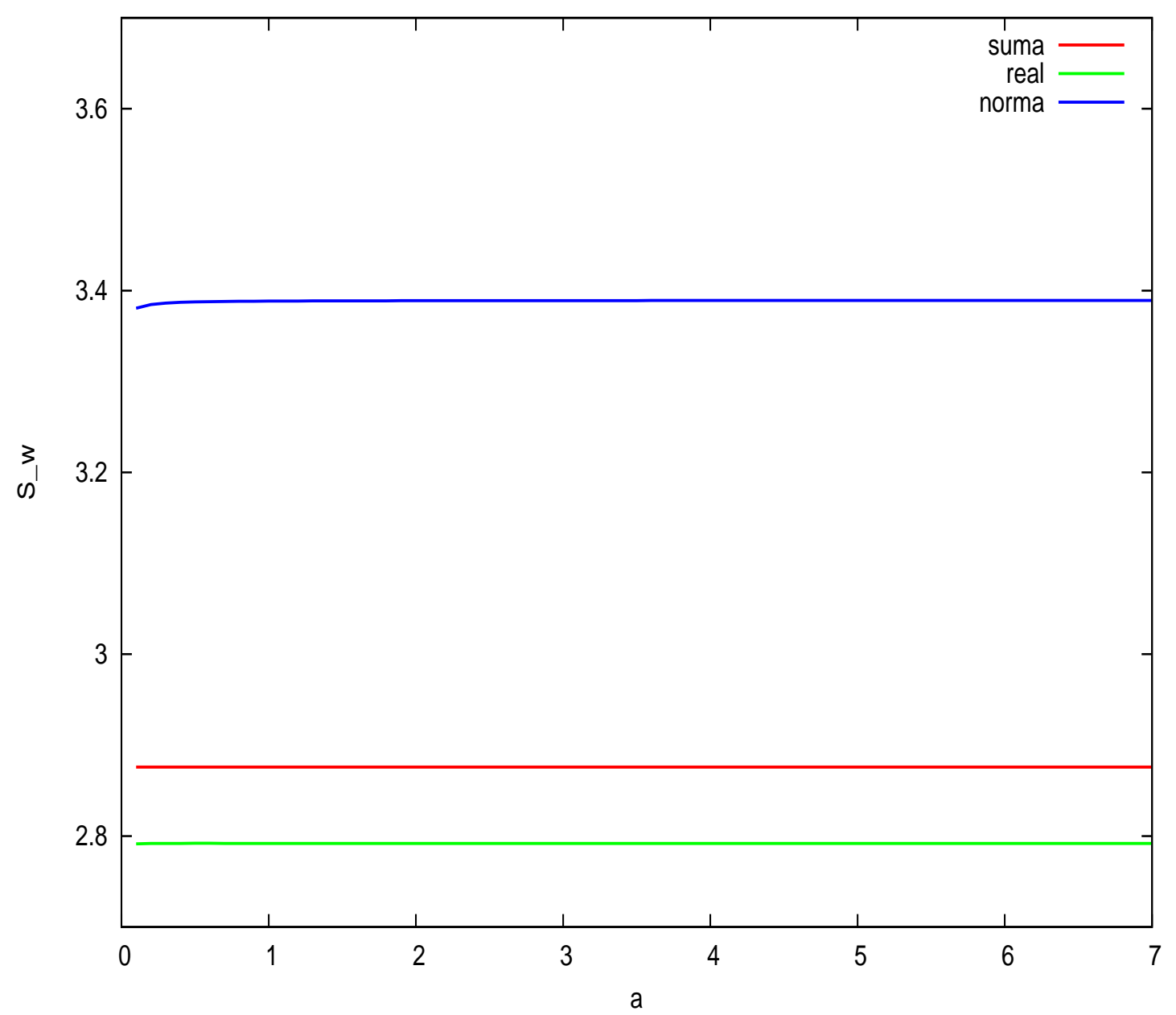

Figura 4.3: Parte real (verde) y norma (azul) de la entropía de Shannon de la función de Wigner, suma entrópica, $s_{\psi}+s_{\widetilde{\psi}}$, (rojo). Comportamiento con $a$ para $n=5$ 


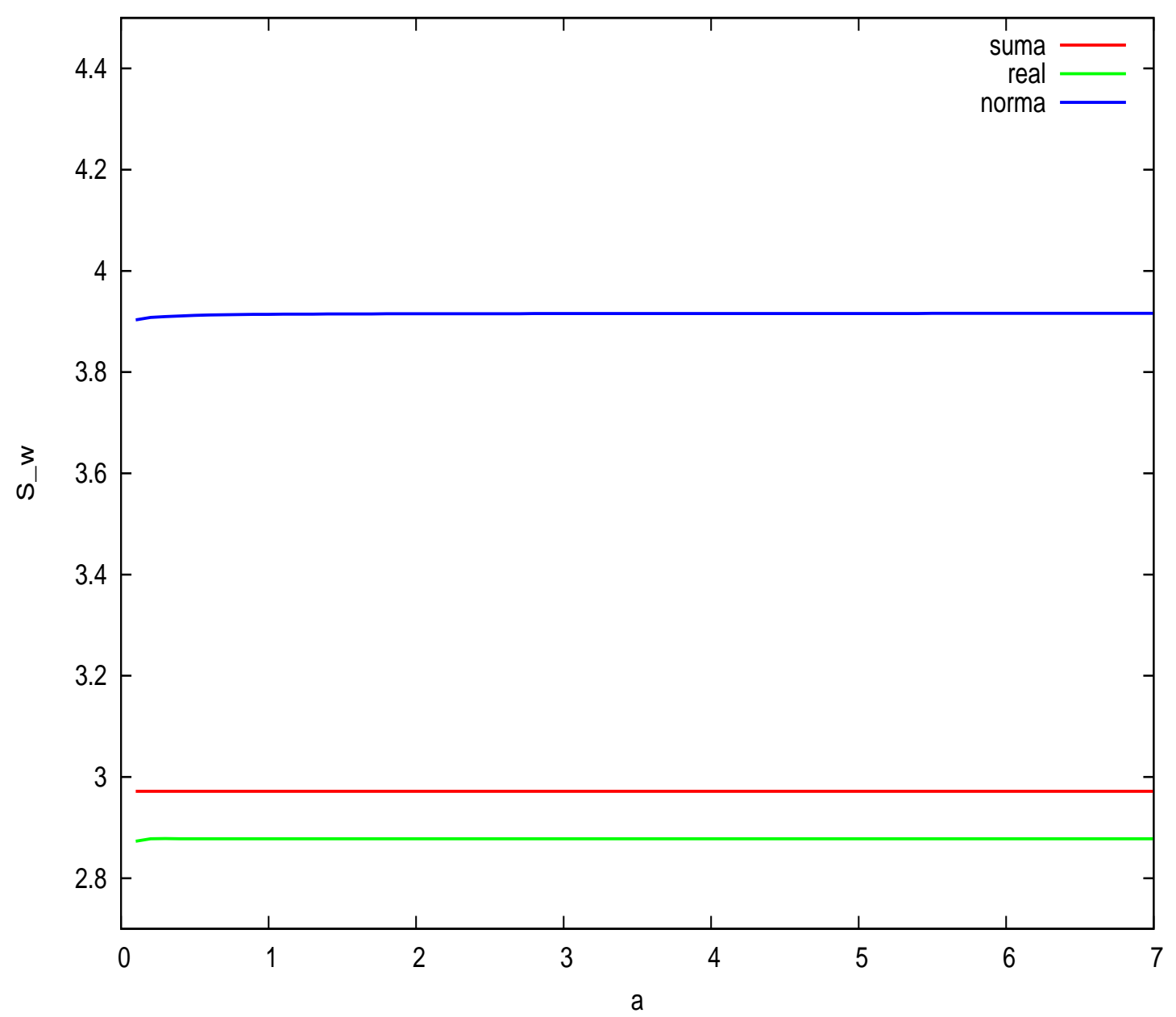

Figura 4.4: Parte real (verde) y norma (azul) de la entropía de Shannon de la función de Wigner, suma entrópica, $s_{\psi}+s_{\widetilde{\psi}}$, (rojo). Comportamiento con $a$ para $n=10$ 
alización natural de esta entropía lo constituye la entropía de Shannon de la función de Wigner.

En la suma entrópica está contenida la información sobre el principio de incertidumbre, es decir la correlación posición-momento proveniente de la no-conmutatividad. En la entropía de Shannon de la función de Wigner parte de esta información del principio de incertidumbre se encuentra contenida en las regiones negativas.

En el estado basal $(n=1), s_{\psi}+s_{\widetilde{\psi}}>\left|s_{w}\right|>\operatorname{Re}\left[s_{w}\right]$. La distribución separable (producto de las densidades) está más deslocalizada que la función de Wigner, sea que la deslocalización se mida en ésta última con la norma o con la parte real de la entropía de Shannon.

En el primer estado excitado $(n=2),\left|s_{w}\right|>\operatorname{Re}\left[s_{w}\right]>s_{\psi}+s_{\widetilde{\psi}}$. En este caso la distribución que es un producto de las densidades es más localizada que la función de Wigner, sea que la localización se mida con la parte real o con la norma de su entropía.

Con $n=5$ y $n=10,\left|s_{w}\right|>s_{\psi}+s_{\widetilde{\psi}}>\operatorname{Re}\left[s_{w}\right]$. De hecho esto ocurre para $n=3, \ldots, 10$, figura (4.5). Puede inferirse que en estados de más alta energía la distribución separable estás más localizada que la función de Wigner si medimos la localización en ésta última utilizando la norma de su entropía, pero está más deslocalizada si utilizamos la parte real.

Estos comportamientos tienen impacto en la medición de la correlación por medio de la información mutua.

La figura (4.5) muestra el comportamiento con $n$ de diferentes definiciones posibles de la entropía de Shannon de la función de Wigner y de la 


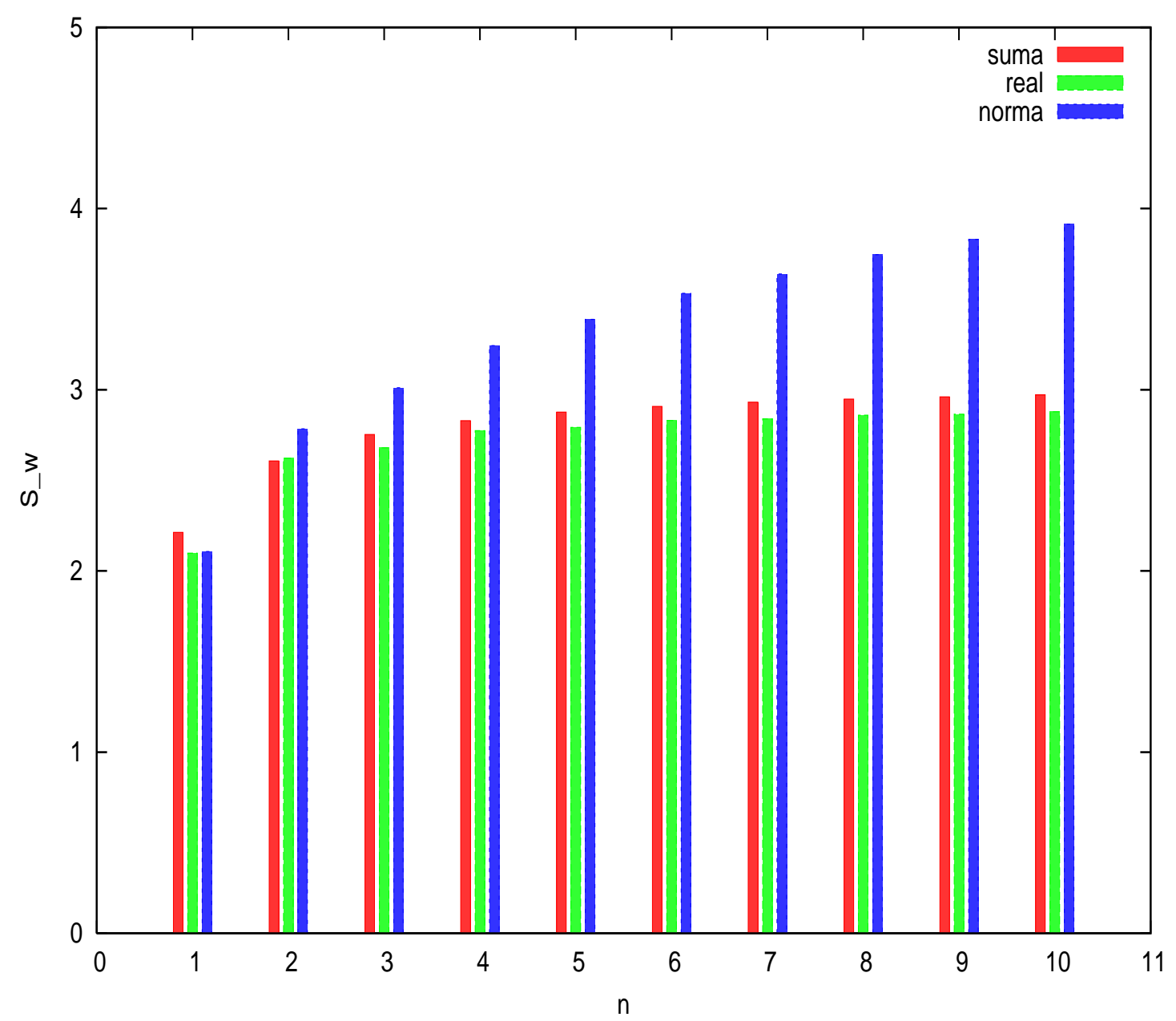

Figura 4.5: Entropía de Shannon de la función de Wigner, se muestra la parte real (verde) y la norma (azul), además de la suma entrópica (rojo). Comportamiento con $n$, para $a=1$. 
suma entrópica. El comportamiento de las tres curvas es consistente, pues en todos los casos la deslocalización se incrementa con la energía.

Conforme $n$ crece, la distancia entre las curvas correspondientes a la parte real y a la norma de la entropía calculada con la función de Wigner aumenta, esto es debido a que las regiones negativas (a las que se debe la parte imaginaria de la entropía) crecen, van haciéndose más importantes (pueden consultarse los valores en el Apéndice B).

La curva correspondiente a la parte real de la entropía de Shannon de la función de Wigner es siempre menor que la curva de la suma entrópica $\left(\operatorname{Re}\left[s_{w}\right]<\right.$ $s_{\psi}+s_{\tilde{\psi}}$ ), salvo en el caso $n=2$. Estas dos curvas son muy similares.

\subsection{Correlación posición-momento.}

En lo que refiere a las medidas de correlación, la covarianza cuántica es cero en este sistema, al igual que las funciones de correlación de orden mayor; esto implica que la covarianza no puede medir la correlación posiciónmomento en este sistema. Esta correlación existe en virtud del principio de incertidumbre y la covarianza es cero por la simetría del problema, pues en cada punto de la caja existe la misma probabilidad de que la velocidad de la partícula sea positiva o negativa, por tanto $<\hat{p}>=<\hat{x} \hat{p}>=0$, lo que implica que $\operatorname{cov}(\hat{x}, \hat{p})=0$.

La figura (4.6) muestra diferentes posibilidades para definir la información mutua como función de $n$. Discutiremos algunos aspectos de las diferentes curvas. 


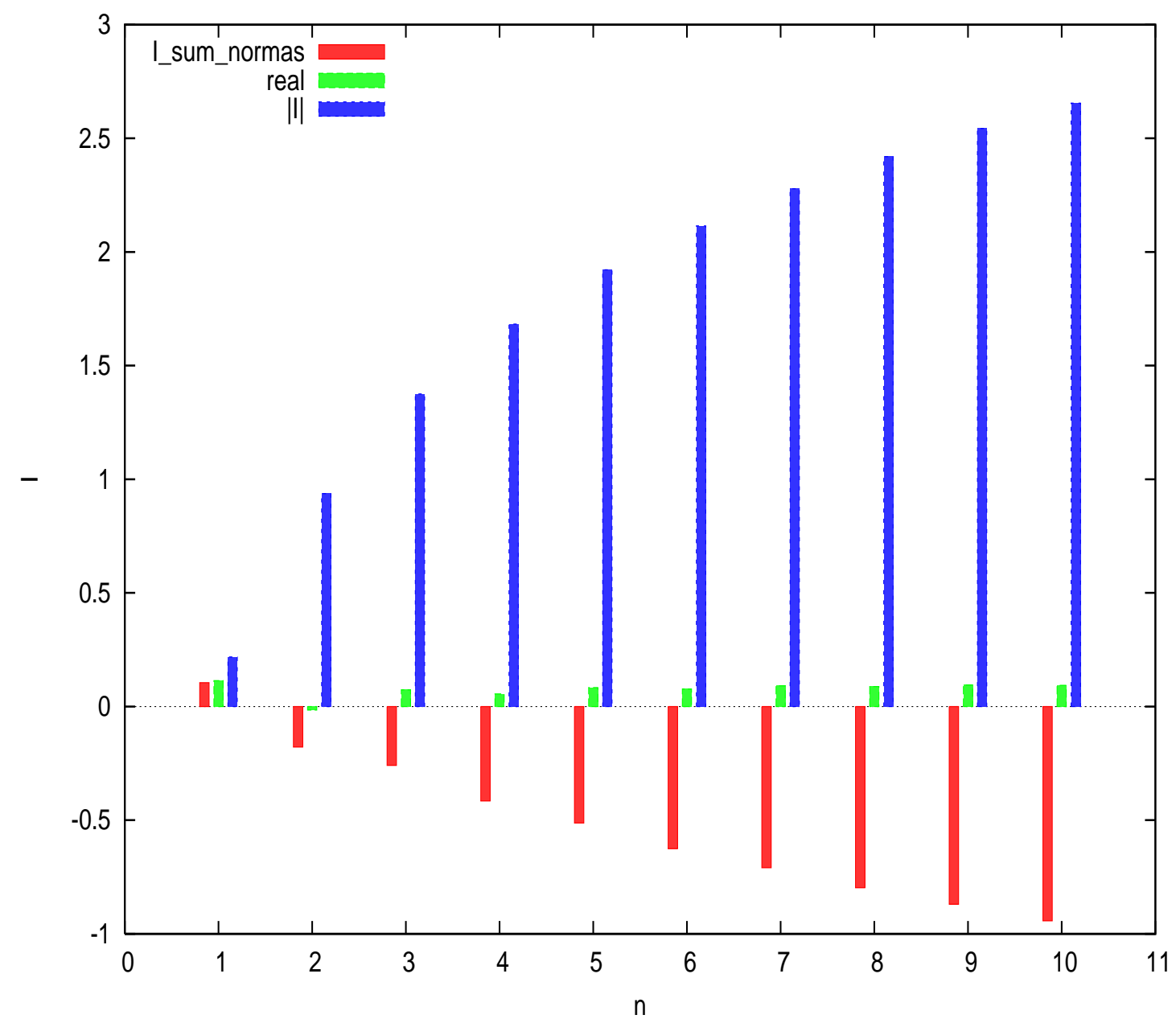

Figura 4.6: Información mutua calculada con la función de Wigner. Se muestran en la gráfica las curvas correspondientes a $\left|s_{\psi}\right|+\left|s_{\widetilde{\psi}}\right|-\left|s_{W}\right|$ (rojo); a $\operatorname{Re}\left[s_{\psi}+s_{\widetilde{\psi}}-s_{W}\right]$ (verde); y a $\left|s_{\psi}+s_{\widetilde{\psi}}-s_{W}\right|$ (azul), con respecto a $n$. 
En el estado basal, cualquiera que sea la definición de la información mutua, siempre es positiva. En el primer estado excitado, la información mutua es negativa, salvo en el caso de considerar la norma, que por definición es siempre positiva.

La curva que corresponde a $\left|s_{\psi}+s_{\widetilde{\psi}}-s_{W}\right|$ es monótonamente creciente con la energía, este comportamiento es consistente con la idea de que la correlación posición-momento en los sistemas clásicos es siempre mayor que en los sistemas cuánticos [52], pues en una interpretación del principio de correspondencia de Bohr, la mecánica cuántica debe reducir a la mecánica clásica para números cuánticos muy grandes.

Es muy notorio que conforme $n$ crece esta curva va separándose cada vez más de la curva que representa la parte real de la información mutua, lo que implica que las regiones negativas son cada vez más importantes, pues son las que provocan la parte imaginaria (véase el Apéndice B para detalles de los valores). En este caso hemos impuesto por la definición de la norma que la información mutua sea siempre positiva.

La curva que corresponde a $\operatorname{Re}\left[s_{\psi}+s_{\widetilde{\psi}}-s_{W}\right]$ es mayor que cero en todos los casos, salvo para $n=2$, es relevante que su comportamiento sea casi constante, pues predice que la correlación posición-momento sería similar para todos los estados graficados de la partícula-en-una-caja. Debe notarse también que, en general, esta curva es la que predice la menor correlación entre la posición y el momento (véase el Apéndice B para detalles de los valores).

Por otro lado, para analizar la curva que corresponde a $\left|s_{\psi}\right|+\left|s_{\widetilde{\psi}}\right|-\left|s_{W}\right|$, 
es importante escribir la definición de la información mutua en términos de las entropías condicionales. Para una distribución que cumple todos los axiomas de la teoría de la probabilidad, digamos $f(x, y)$, la información mutua puede escribirse,

$$
\begin{array}{r}
I_{x, y}=\int d x d y f(x, y) \ln \frac{f(x, y)}{f_{1}(x) f_{2}(y)}=s_{x}+s_{y}-s_{x, y} \\
=s_{x}-s(x \mid y)=s_{y}-s(y \mid x)
\end{array}
$$

donde $f_{1}(x)$ y $f_{2}(y)$ son las marginales de $f(x, y), \mathrm{y}$,

$$
s(x \mid y)=-\int d x d y f(x, y) \ln \frac{f(x, y)}{f_{2}(y)}
$$

es la entropía de $x$ (su incertidumbre) una vez que conocemos $y$, así como,

$$
s(y \mid x)=-\int d x d y f(x, y) \ln \frac{f(x, y)}{f_{1}(x)}
$$

es la entropía de $y$ una vez que conocemos $x$; la incertidumbre de $y$ una vez conocida $x$.

La subaditividad de la entropía conjunta proviene de que la distribución $f(x, y)$ es positivo-definida e impone que,

$$
s(x \mid y) \leq s_{x}
$$

y que,

$$
s(y \mid x) \leq s_{y},
$$


lo cual puede enunciarse como "el conocimiento de una variable sólo puede provocar una disminución en la incertidumbre de la otra variable". Este es un resultado obvio en la teoría clásica de la probabilidad, pero encuentra un problema si las variables son conjugadas según la mecánica cuántica, pues la relación entre éstas por causa del principio de incertidumbre tendría que enunciarse como sigue: "el conocimiento de una variable provoca un aumento en la incertidumbre de la variable conjugada".

Este último enunciado es ilustrado por la curva $\left|s_{\psi}\right|+\left|s_{\widetilde{\psi}}\right|-\left|s_{W}\right|$, pues es negativa en todos los estados graficados, salvo en el basal. Si definimos las entropías conjuntas entre posición y momento como,

$$
s(x \mid p)=-\int d x d p W(x, p) \ln \frac{W(x, p)}{|\widetilde{\psi}(p)|^{2}}
$$

$\mathrm{y}$

$$
s(p \mid x)=-\int d x d p W(x, p) \ln \frac{W(x, p)}{|\psi(x)|^{2}}
$$

tenemos que concluir que en los estados donde es negativa la información mutua, que puede definirse como,

$$
I=s_{\psi}-s(x \mid p)=s_{\widetilde{\psi}}-s(p \mid x)
$$

ocurre que,

$$
s(x \mid p)>s_{\psi}
$$

o bien, 


$$
s(p \mid x)>s_{\widetilde{\psi}}
$$

lo que implica que "conocer $x$ (localización de esa densidad) implica aumentar la incertidumbre en $p$ (deslocalizar esa densidad), y viceversa", un enunciado acorde con el principio de incertidumbre.

Por otro lado, si tomamos el cero como la referencia de no-correlación, como en el caso del coeficiente de correlación, podemos argumentar que esta curva predice que la correlación aumenta con la energía, un resultado consistente con la idea de que la correlación posición-momento en sistemas clásicos es mayor que en sistemas cuánticos y que la mecánica cuántica debe reducir a la mecánica clásica para números cuánticos grandes.

Los valores negativos son familiares en otras medidas como en el caso del coeficiente de correlación $(\tau)$, donde ocurre, $-1 \leq \tau \leq+1$. 


\section{Dos-partículas-en-una-caja}

En el capítulo anterior hemos estudiado la relación de incertidumbre entrópica para una-partícula-en-una-caja, siguiendo los trabajos de Majernik, et. al. $[8,9]$, hemos extendido este análisis al considerar el efecto del confinamiento sobre las entropías informacionales en ambos espacios y en espacio-fase, y hemos estudiado la correlación posición-momento a través de la definición de la entropía de Shannon de la función de Wigner, una generalización de la suma entrópica vista como la entropía de una distribución en espacio-fase que es el producto de las densidades en cada espacio, véase Ec. (2.10).

Estamos interesados en extender el análisis al modelo de dos partículas sin interacción y sin espín en una caja unidimensional, estudiando primero la localización de las distribuciones de pares y sus marginales como función de $n_{2}$. Pondremos atención enseguida a la correlación debida a la indistinguibilidad de las partículas estudiándola con la información mutua en los espacios de posición y de momento, también como función de $n_{2}$.

Después analizaremos el efecto del confinamiento en las entropías de las marginales y en las entropías de pares, en la suma entrópica al nivel de dos 
partículas, y en la información mutua en ambos espacios, es decir haremos el análisis del comportamiento de las cantidades como función del tamaño de la caja, $a$.

Enseguida saltamos a la discusión de la correlación posición-momento en este modelo definiendo informaciones mutuas de órdenes mayores y discutiendo su relación.

\subsection{Localización y correlación debidas a la indistinguibilidad como funciones de $n_{2}$.}

Debido a que las partículas no interactúan, pueden construirse tres funciones de onda en el espacio de posiciones, atendiendo a la simetría, a partir de la función de onda de una-partícula-en-una-caja [10],

$$
\begin{gathered}
\boldsymbol{\Psi}_{\text {nosim }}=\psi_{1}\left(x_{1}\right) \psi_{2}\left(x_{2}\right) \\
\boldsymbol{\Psi}_{\text {antisim }}=\frac{1}{\sqrt{2}}\left[\psi_{1}\left(x_{1}\right) \psi_{2}\left(x_{2}\right)-\psi_{2}\left(x_{1}\right) \psi_{1}\left(x_{2}\right)\right]
\end{gathered}
$$

$\mathrm{y}$

$$
\boldsymbol{\Psi}_{\text {sim }}=\frac{1}{\sqrt{2}}\left[\psi_{1}\left(x_{1}\right) \psi_{2}\left(x_{2}\right)+\psi_{2}\left(x_{1}\right) \psi_{1}\left(x_{2}\right)\right],
$$

y las correspondientes tres funciones de onda en el espacio de momentos,

$$
\widetilde{\boldsymbol{\Psi}}_{\text {nosim }}=\widetilde{\psi}_{1}\left(p_{1}\right) \widetilde{\psi}_{2}\left(p_{2}\right),
$$




$$
\widetilde{\boldsymbol{\Psi}}_{\text {antisim }}=\frac{1}{\sqrt{2}}\left[\widetilde{\psi}_{1}\left(p_{1}\right) \widetilde{\psi}_{2}\left(p_{2}\right)-\widetilde{\psi}_{2}\left(p_{1}\right) \widetilde{\psi}_{1}\left(p_{2}\right)\right]
$$

y

$$
\widetilde{\boldsymbol{\Psi}}_{\text {sim }}=\frac{1}{\sqrt{2}}\left[\widetilde{\psi}_{1}\left(p_{1}\right) \widetilde{\psi}_{2}\left(p_{2}\right)+\widetilde{\psi}_{2}\left(p_{1}\right) \widetilde{\psi}_{1}\left(p_{2}\right)\right] .
$$

Arriba, nosim se refiere a "no simétrica" (y corresponde a dos partículas distinguibles), y antisim a "antisimétrica" y sim a "simétrica" (las cuales corresponden a dos partículas indistinguibles). Hacemos notar que debido a la ortogonalidad de los estados, las marginales de las funciones simétrica y antisimétrica son iguales, este es un ejemplo en que dos funciones de onda diferentes generan la misma densidad de una partícula.

Las entropías de las marginales y las de pares fueron calculadas usando una cuadratura de Gauss-Legendre de 12 puntos en cada subintervalo y trabajando con precisión doble, el esquema fue probado calculando las normalizaciones.

\subsubsection{Entropía de Shannon de las marginales.}

Las figuras (5.1), (5.2) muestran las gráficas de las entropías de las marginales en los espacios de posición y momento, puede observarse que la densidad de una partícula que proviene de la función de onda de dos partículas indistinguibles (antisimétrica y simétrica) está más deslocalizada que la densidad de una partícula que proviene de la función de onda de dos partículas distinguibles (no simétrica), en ambos espacios. La indistinguibilidad implica 


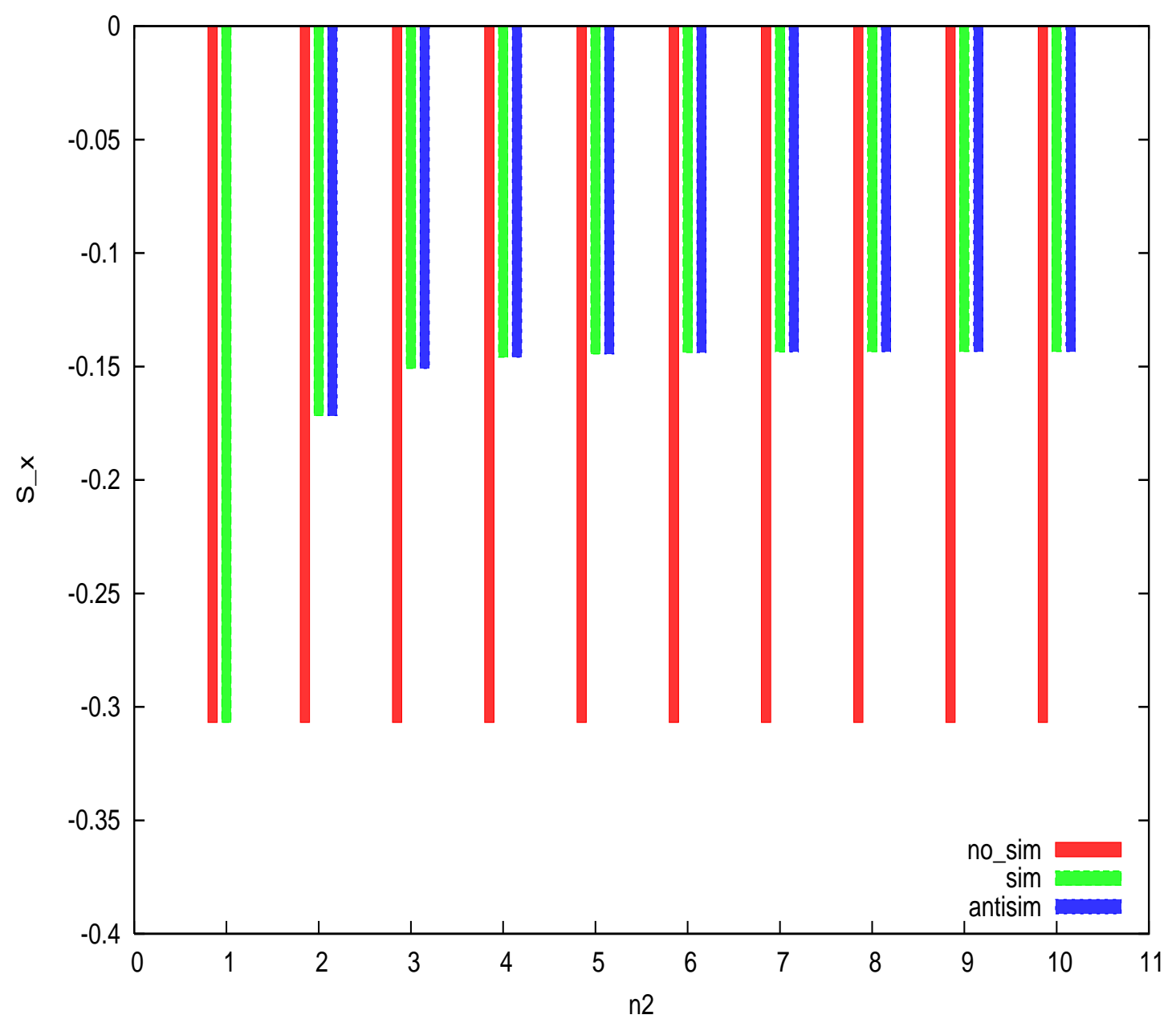

Figura 5.1: Entropías de Shannon de las marginales de la función de onda de dos partículas en el espacio de posición, con $n_{1}=1$ y $a=1$. 


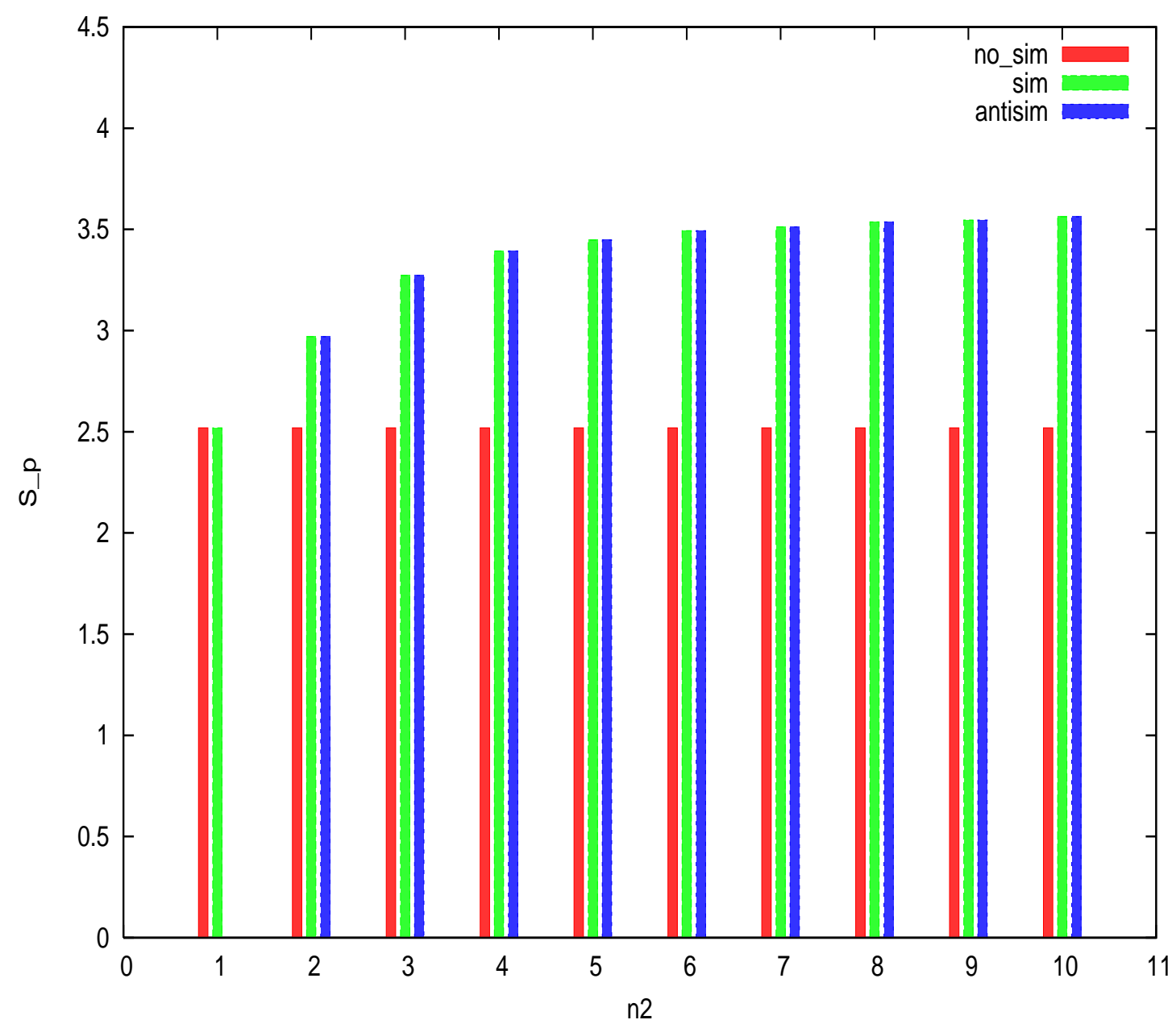

Figura 5.2: Entropías de Shannon de las marginales de la función de onda de dos partículas en el espacio de momento, con $n_{1}=1$ y $a=1$. 


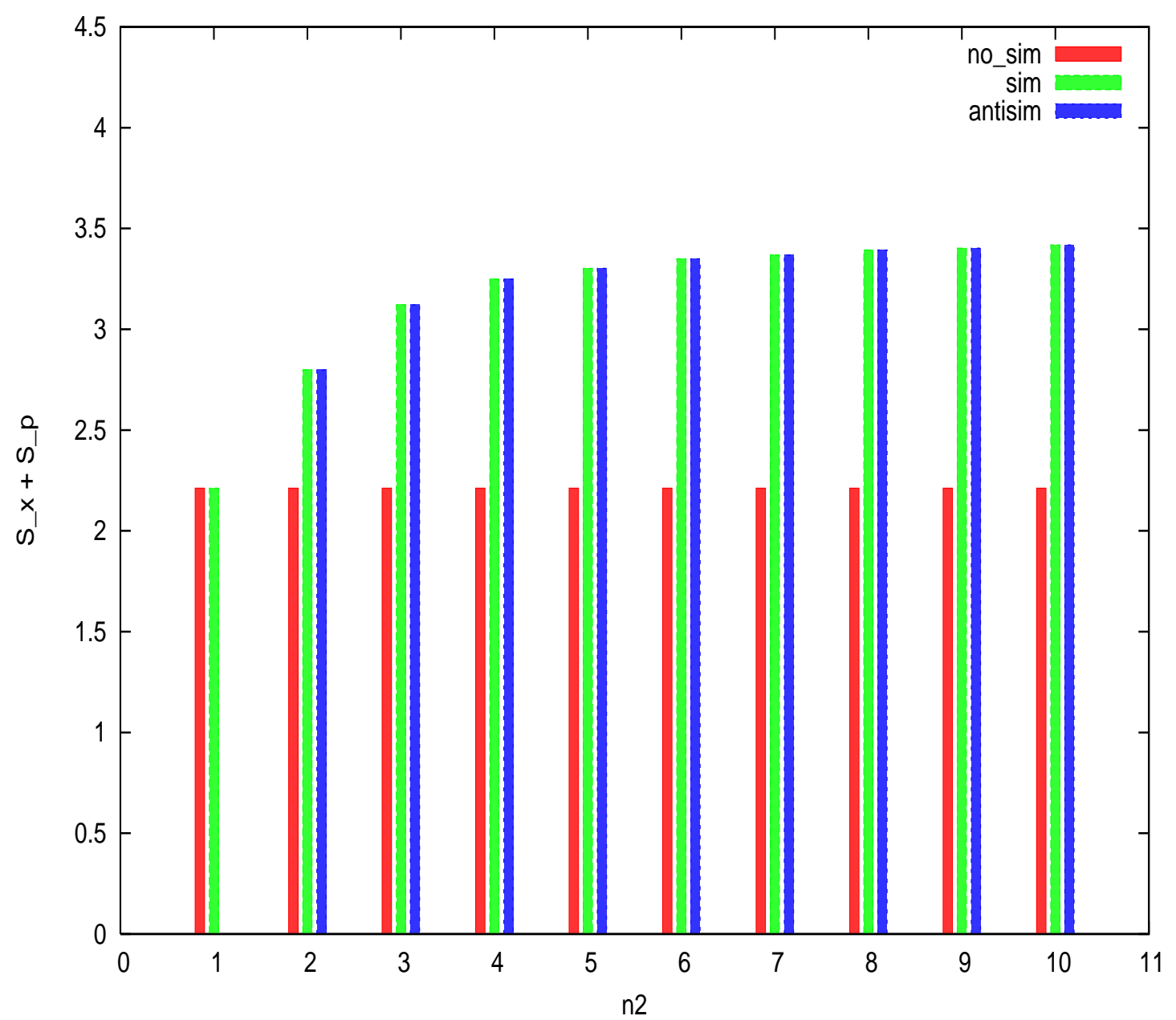

Figura 5.3: Suma entrópica de las marginales de la función de onda de dos partículas, con $n_{1}=1$ y $a=1$. 
mayor deslocalización en el nivel de una partícula.

Resalta también que en ambos espacios, las densidades que provienen de la función de dos partículas indistinguibles se deslocalizan mientras crece $n_{2}$. Para la densidad que proviene de la función de dos partículas distinguibles el comportamiento es constante porque hemos hecho la integración sobre $x_{2}$ o $p_{2}$, según corresponda, y hemos graficado contra $n_{2}$ fijando $n_{1}=1$.

La deslocalización es mayor en el espacio de momento que en el de posición para todos los casos mostrados.

La figura (5.3) muestra la suma entrópica de las marginales, todas las sumas entrópicas cumplen con la cota establecida por el principio de incertidumbre, Ec. (2.7). La tendencia de la suma entrópica de las partículas indistinguibles es la misma que la de la suma entrópica para una-partículaen-una-caja.

\subsubsection{Entropía de Shannon de la densidad de pares.}

Para estudiar las densidades de pares, pueden definirse las entropías de Shannon de pares,

$$
\begin{gathered}
s_{\Psi}=-\int d x_{1} d x_{2}\left|\boldsymbol{\Psi}\left(x_{1}, x_{2}\right)\right|^{2} \ln \left|\boldsymbol{\Psi}\left(x_{1}, x_{2}\right)\right|^{2}, \\
s_{\widetilde{\boldsymbol{\Psi}}}=-\int d p_{1} d p_{2}\left|\widetilde{\boldsymbol{\Psi}}\left(p_{1}, p_{2}\right)\right|^{2} \ln \left|\widetilde{\boldsymbol{\Psi}}\left(p_{1}, p_{2}\right)\right|^{2} .
\end{gathered}
$$

También puede formularse una relación de incertidumbre entrópica al nivel de dos partículas [16], 


$$
s_{\Psi}+s_{\widetilde{\Psi}} \geq 2(1+\ln \pi) .
$$

Las figuras (5.4), (5.5) muestran el comportamiento de las entropías de pares en los espacios de posición y momento para las diferentes simetrías. En todos los casos la distribución se encuentra más localizada en el espacio de posición que en el de momento, esto se debe a que hemos impuesto que se localice la distribución en el espacio de posición al fijar $a=1$, lo cual provoca que la velocidad aumente y se deslocalice la distribución de momentos. En los casos en que $n_{2}$ es par, las entropías de pares de las funciones simétrica y antisimétrica son iguales.

En el espacio de momento la deslocalización de la distribución aumenta con $n_{2}$, esto ocurre porque la energía es puramente cinética y conforme vamos a estados excitados provocamos que el valor medio de la velocidad aumente y crezca $s_{\widetilde{\Psi}}$.

En el nivel de dos partículas, las densidades que provienen de la función de onda de las partículas distinguibles se encuentran más deslocalizadas que las que provienen de la función de onda de las partículas indistinguibles en el espacio de posición, mientras que en el espacio de momento ocurre lo contrario. Puede argumentarse que este comportamiento refleja la información del principio de incertidumbre, pues si tenemos como referencia la distribución distinguible, la simetrización de la función de onda provoca la localización de las distribuciones en el espacio de posición, lo que implica que la distribución en el espacio de momento debe deslocalizarse. 


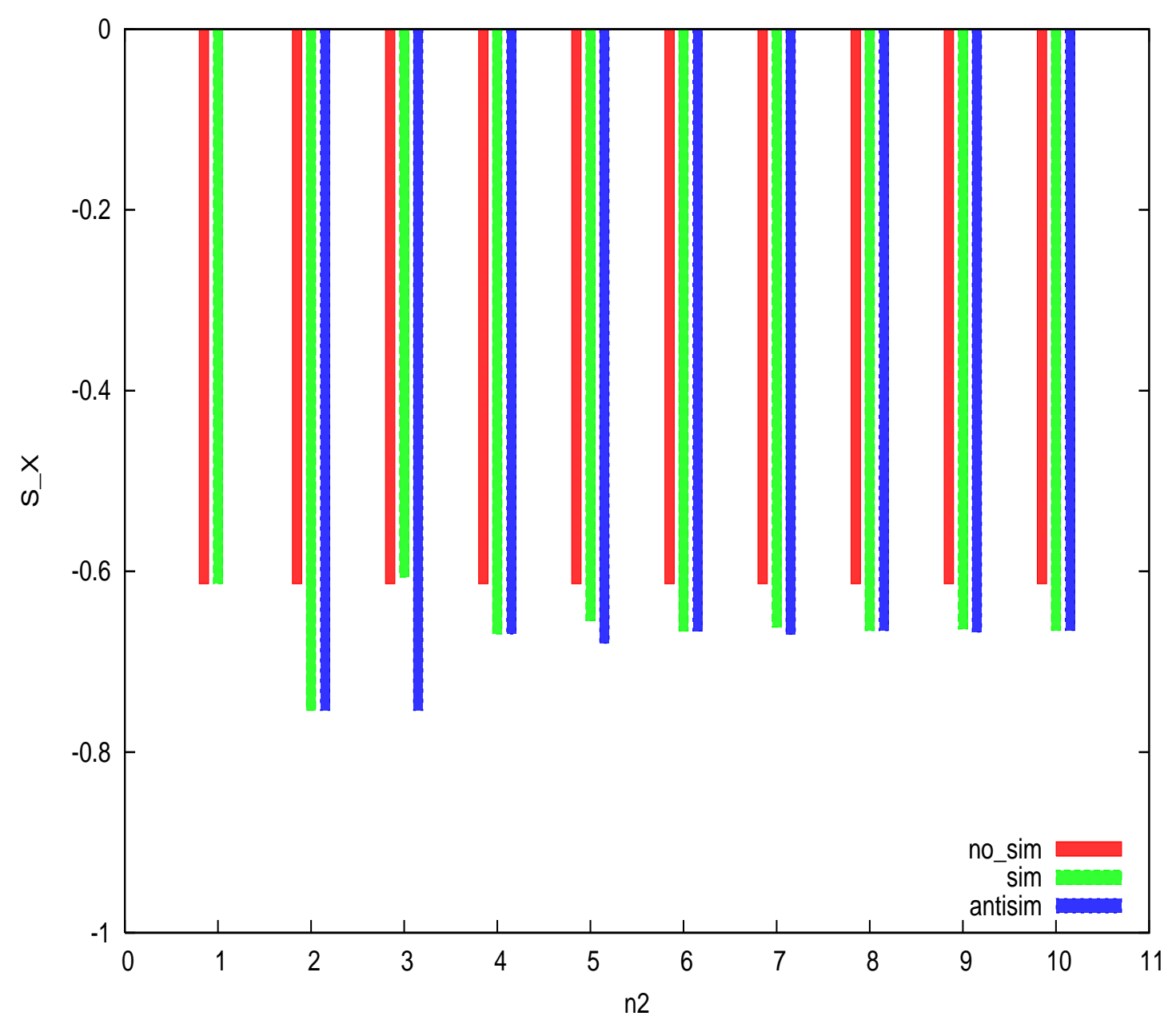

Figura 5.4: Entropía de Shannon de la función de dos partículas en el espacio de posición, con $n_{1}=1$ y $a=1$. 


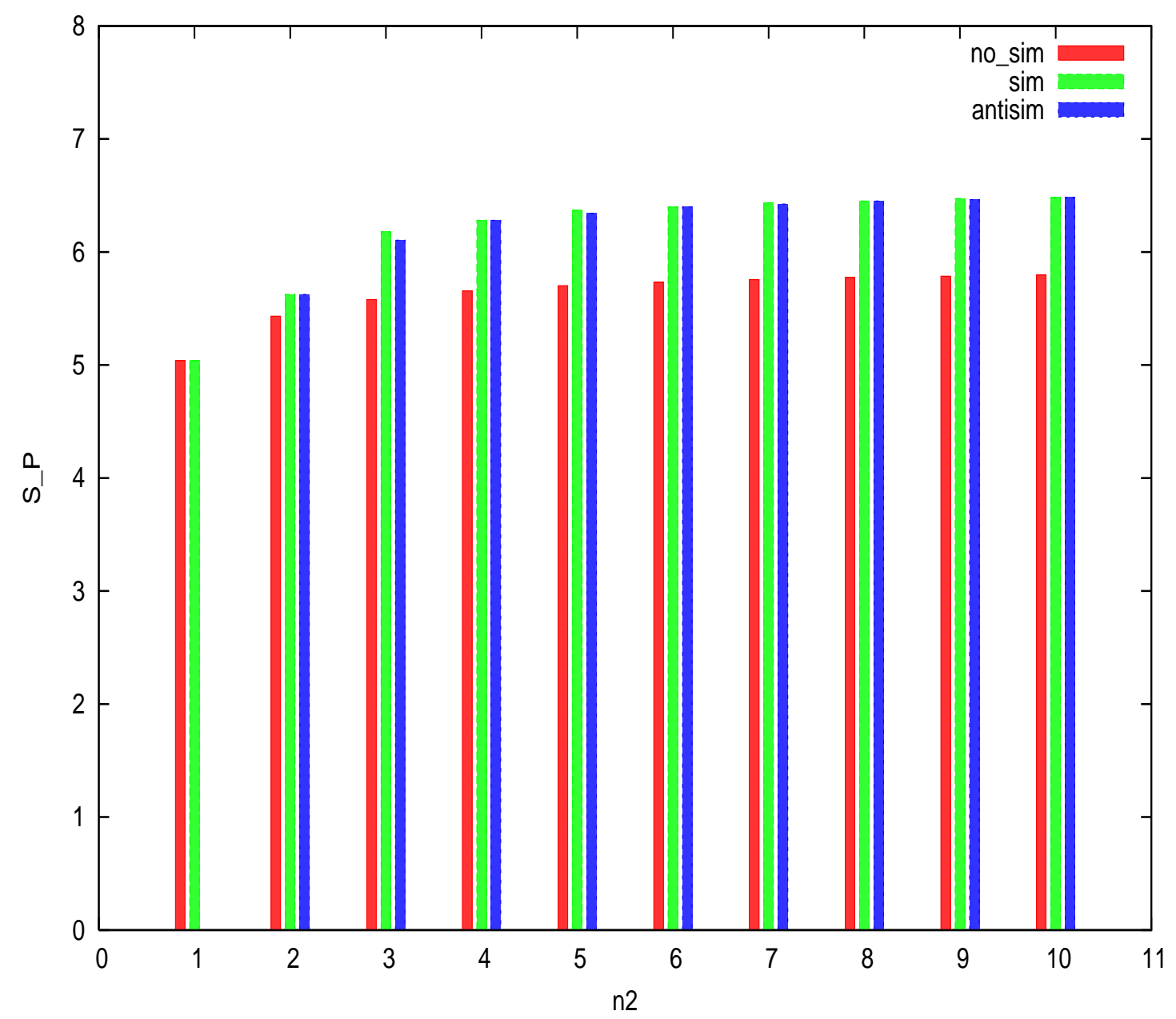

Figura 5.5: Entropía de Shannon de la función de dos partículas en el espacio de momento, con $n_{1}=1$ y $a=1$. 


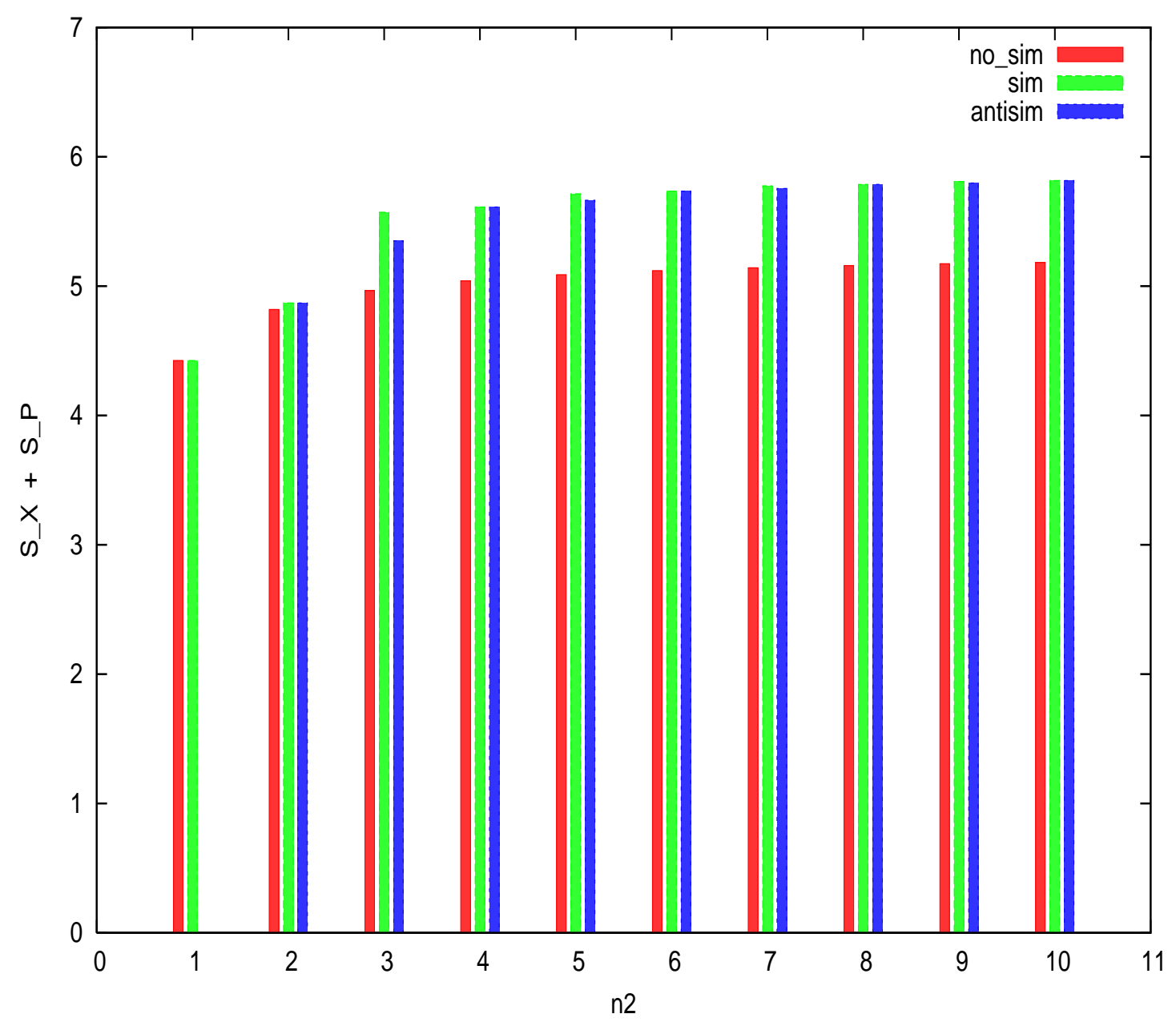

Figura 5.6: Suma entrópica de la función de dos partículas, con $n_{1}=1 \mathrm{y}$ $a=1$. 
Es interesante notar que para decidir si las distribuciones asociadas a las partículas indistinguibles están más localizadas que las asociadas a las partículas distinguibles o si es al contrario, la información que proviene del nivel de una partícula (donde las indistinguibles son más deslocalizadas que las distinguibles en ambos espacios) es diferente de la que puede extraerse del análisis en el nivel de dos partículas.

Nuestro sistema son dos partículas y las densidades son analíticas, concluimos entonces que es mejor analizar las entropías de pares. Esta observación es relevante porque a menudo en la mecánica cuántica de átomos y moléculas tratamos con distribuciones reducidas de un electrón, y es factible sugerir que el estudio de las distribuciones de pares puede brindar información que no es visible en el nivel monoelectrónico. Este es posiblemente el caso del enlace químico covalente, que se explica como el fenómeno producido porque dos átomos comparten un par de electrones.

Por otro lado, si se analiza el comportamiento de las entropías de pares de las funciones simetrizadas se observa que la función antisimétrica se encuentra más localizada que la simétrica en ambos espacios. La interpretación física es que en el primer caso la probabilidad de encontrar a una partícula en un punto de la caja influye inversamente en la probabilidad de encontrar ahí mismo a la segunda debido a que no pueden ocupar el mismo lugar en el espacio (por la presencia del hueco de Fermi), es decir que el espacio en que podemos encontrar esta segunda partícula es menor y por tanto su distribución es más localizada; esto no ocurre en el caso de la función simétrica porque no existe tal restricción. 
Conforme $n_{2}$ aumenta, los valores de la entropía de pares de las distribuciones antisimétrica y simétrica van acercándose, esto puede interpretarse teniendo en cuenta que como la energía es puramente cinética, el aumento de $n_{2}$ implica que la velocidad de las partículas es mayor y la probabilidad de encontrarlas en un punto dado disminuye, para la función antisimétrica se sigue que la influencia de la primera partícula sobre la segunda es menor y por eso su entropía va acercándose a la de la distribución simétrica.

El comportamiento de la suma entrópica se muestra en la figura (5.6), y cumple en todos los casos con la cota señalada, Ec. (5.9).

\subsubsection{Información mutua.}

Para el estudio de la correlación entre $\left(x_{1}, x_{2}\right)$ y entre $\left(p_{1}, p_{2}\right)$, definimos las informaciones mutuas en ambos espacios como,

$$
I_{x}=\int d x_{1} d x_{2}\left|\boldsymbol{\Psi}\left(x_{1}, x_{2}\right)\right|^{2} \ln \frac{\left|\boldsymbol{\Psi}\left(x_{1}, x_{2}\right)\right|^{2}}{\left|\psi\left(x_{1}\right)\right|^{2}\left|\psi\left(x_{2}\right)\right|^{2}}=2 s_{\psi}-s_{\Psi}
$$

e

$$
I_{p}=\int d p_{1} d p_{2}\left|\widetilde{\boldsymbol{\Psi}}\left(p_{1}, p_{2}\right)\right|^{2} \ln \frac{\left|\widetilde{\boldsymbol{\Psi}}\left(p_{1}, p_{2}\right)\right|^{2}}{\left|\widetilde{\psi}\left(p_{1}\right)\right|^{2}\left|\widetilde{\psi}\left(p_{2}\right)\right|^{2}}=2 s_{\widetilde{\psi}}-s_{\widetilde{\mathbf{\Psi}}}
$$

Las figuras (5.7) y (5.8) muestran las gráficas de la información mutua en los espacios de posición y momento.

Para $n_{2}$ par, existe la misma correlación en las funciones simétrica y antisimétrica. En los otros casos, la correlación es mayor para la función 


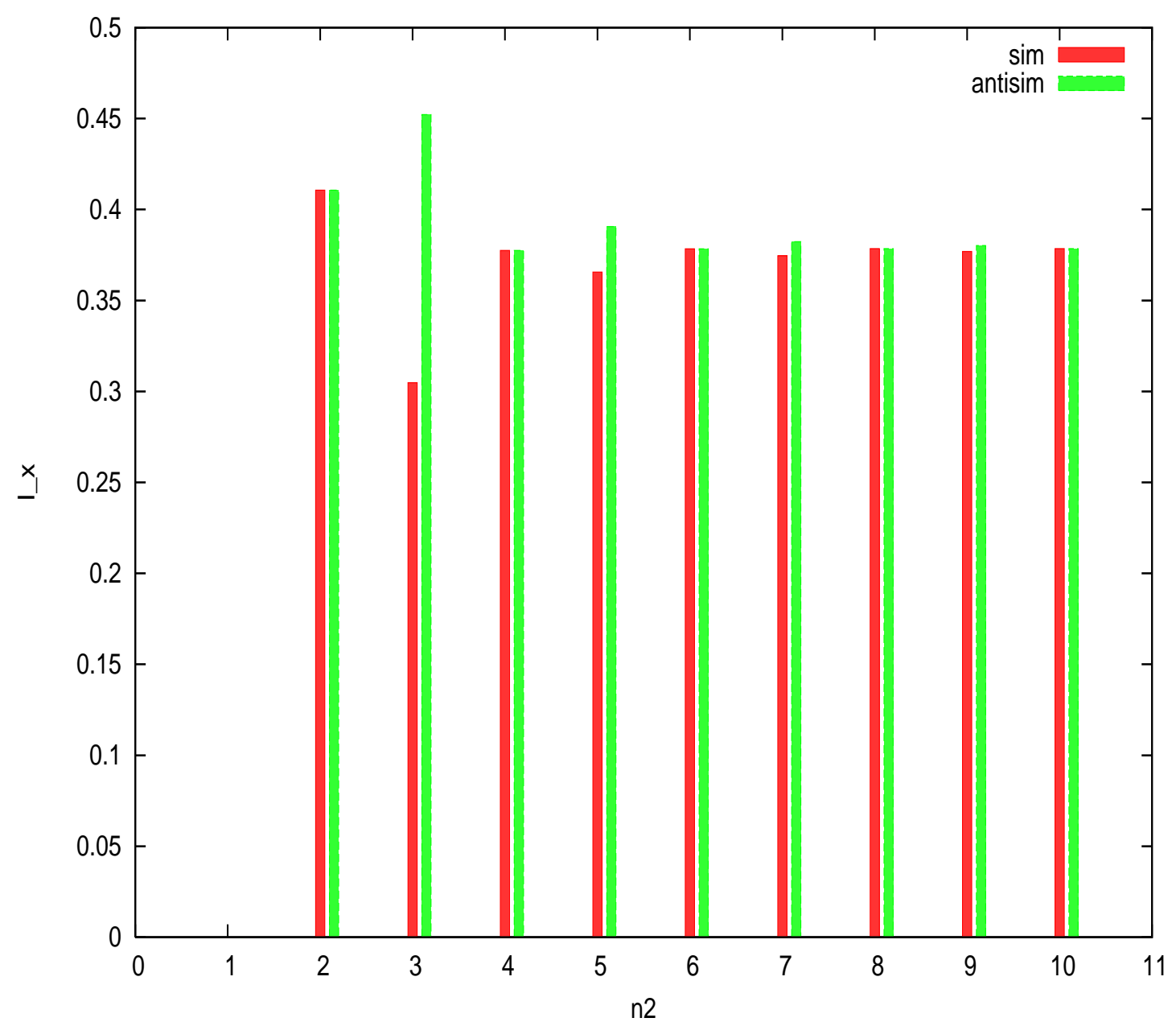

Figura 5.7: Información mutua en el espacio de posición, con $n_{1}=1 \mathrm{y}$ $a=1$. 


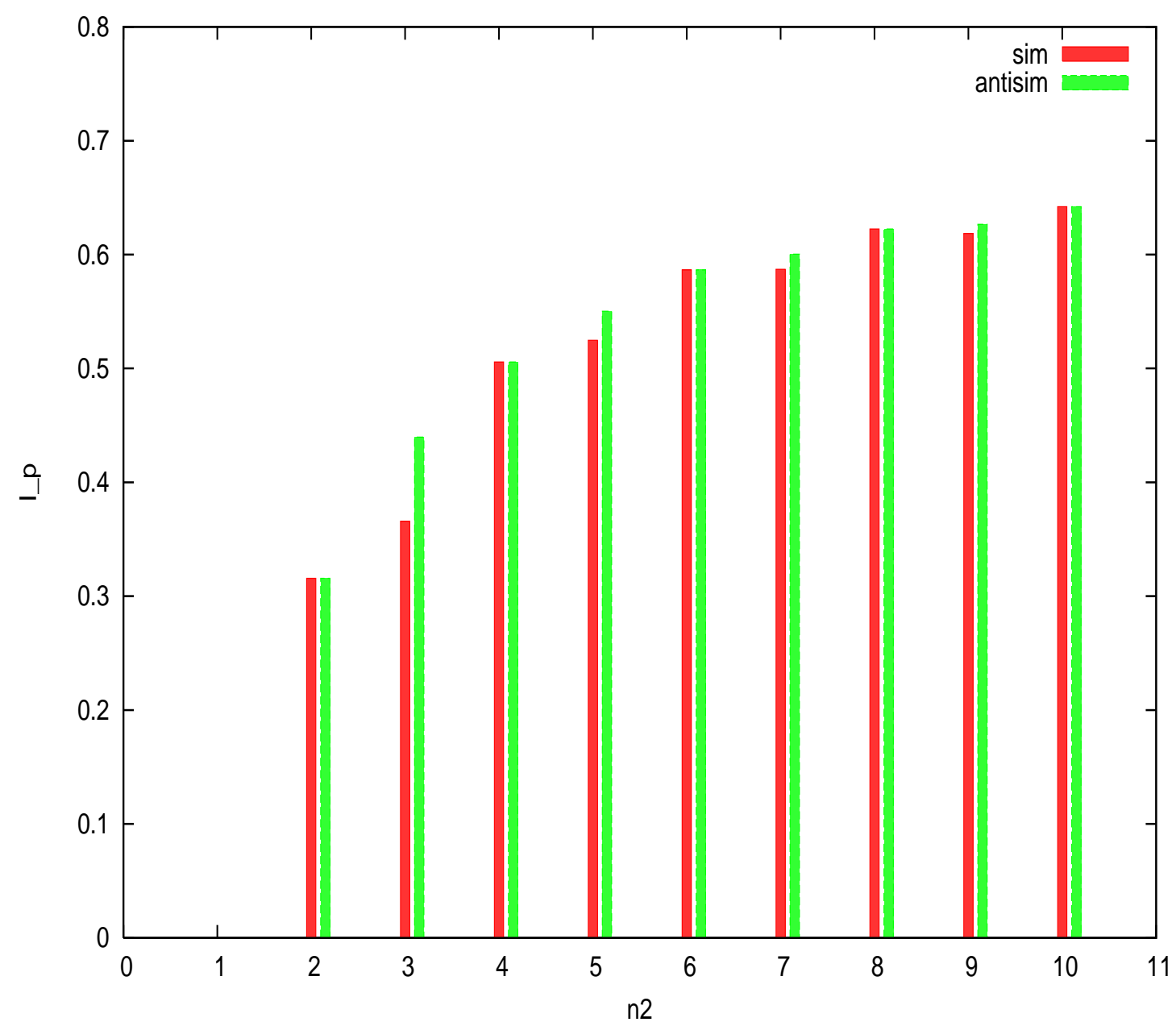

Figura 5.8: Información mutua en el espacio de momento, con $n_{1}=1 \mathrm{y}$ $a=1$. 
antisimétrica que para la función simétrica; este comportamiento puede interpretarse físicamente argumentando que en la función antisimétrica una partícula ejerce una influencia mayor sobre la segunda, debido a la restricción de que ambas no pueden ocupar el mismo punto del espacio (por la presencia del hueco de Fermi), lo cual no ocurre para la función simétrica.

En ambos espacios la correlación asociada a las distribuciones simétrica y antisimétrica va haciéndose similar conforme aumenta la energía. Este comportamiento es consecuente con lo que se ha argumentado arriba para las entropías de pares, pues el aumento en la velocidad implica que en el caso de la función antisimétrica, la influencia de la primera partícula sobre la segunda disminuye.

Otro análisis relevante es el que se sigue de las figuras (5.9) y (5.10), donde se compara la información mutua de cada función en ambos espacios.

La información mutua puede ser vista como una medida de la diferencia entre la localización de la distribución en los niveles de una $\left(s_{\psi}\right.$ o $\left.s_{\widetilde{\psi}}\right)$ y dos partículas $\left(\begin{array}{lll}s_{\Psi} & \mathrm{O} & s_{\widetilde{\boldsymbol{\Psi}}}\end{array}\right)$. Por otro lado, la localización de una distribución habla de un fenómeno llamado condensación. Para el análisis que sigue si la información mutua es mayor en alguno de los espacios puede argumentarse que existe una mayor condensación en éste, esto es importante porque en la naturaleza existen múltiples fenómenos que han sido asociados con una cierta condensación en alguno de los espacios y la información mutua puede emplearse para medirla.

Para la función antisimétrica, la correlación es mayor en el espacio de posición en el estado basal y en el estado excitado $n_{2}=3$, en el resto de los 


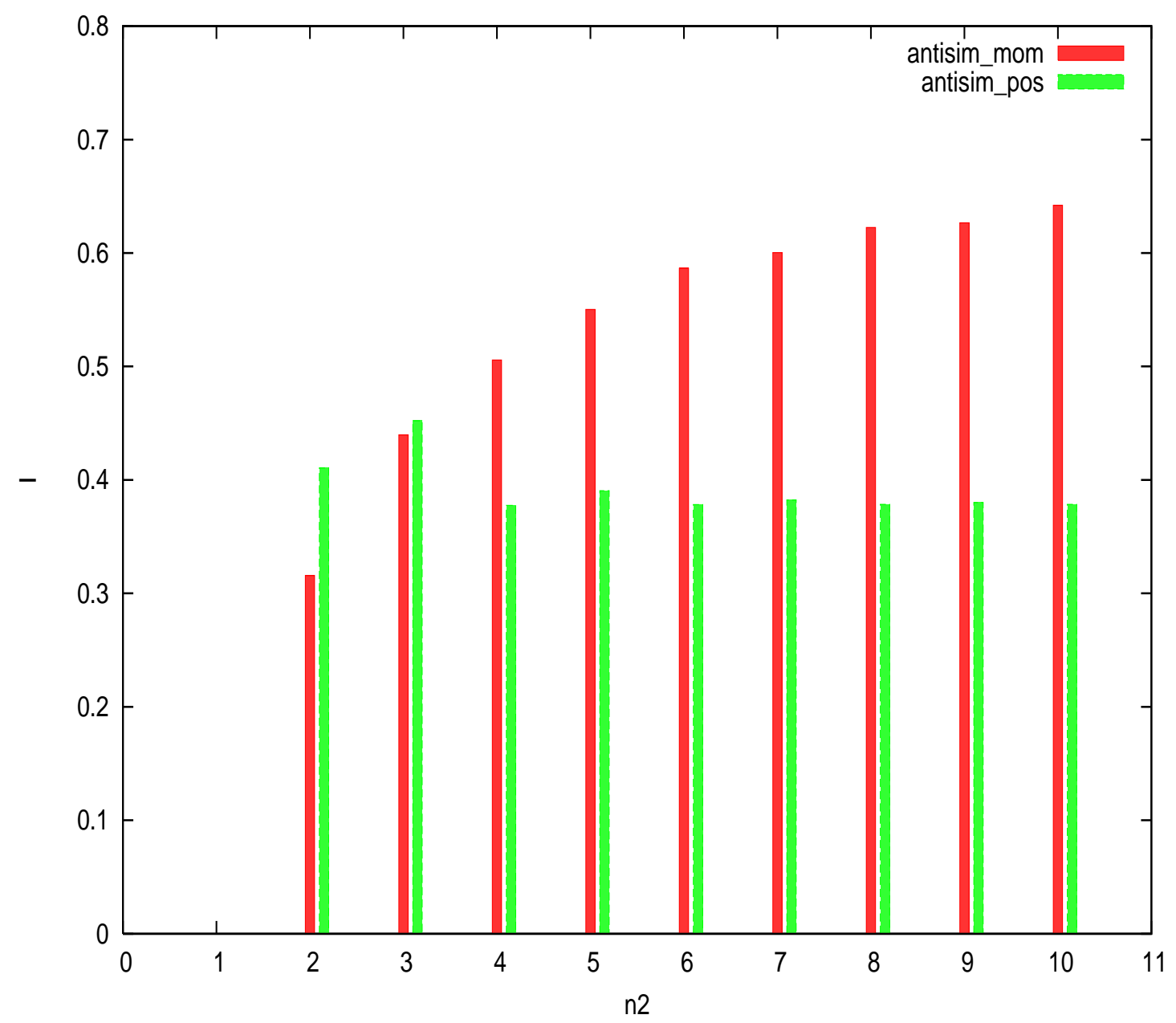

Figura 5.9: Información mutua de la función antisimétrica en ambos espacios, con $n_{1}=1$ y $a=1$. 


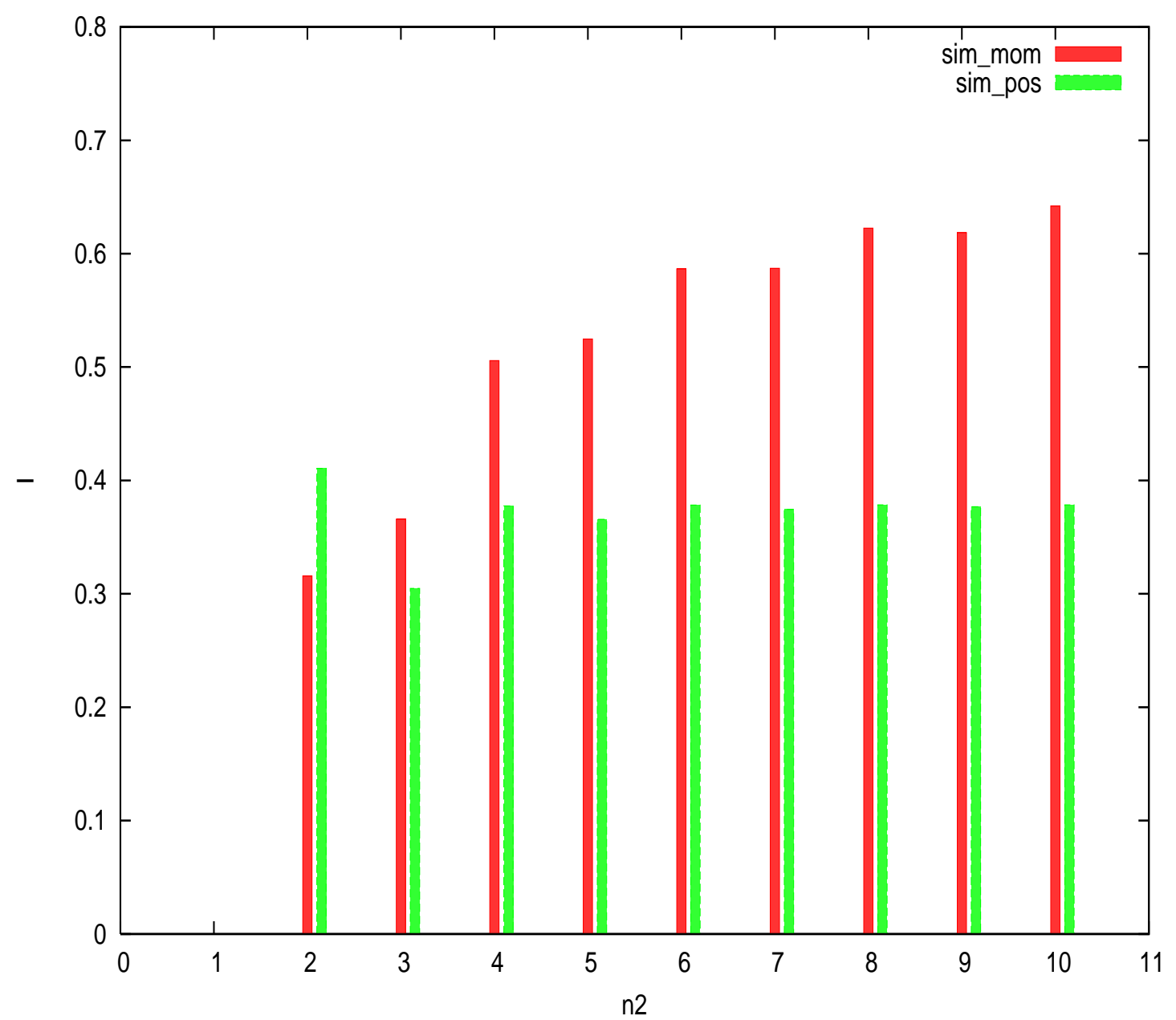

Figura 5.10: Información mutua de las función simétrica en ambos espacios, con $n_{1}=1$ y $a=1$. 
casos graficados es mayor en el espacio de momento.

Para la función simétrica la correlación es mayor en el espacio de posición sólo en el estado basal, siendo mayor en el espacio de momento en el resto de los casos graficados.

Es interesante que en ambas funciones la correlación en el espacio de momento aumenta con $n_{2}$ de manera mucho más pronunciada que en el espacio de posición.

\subsection{Efectos del confinamiento sobre las en- tropías informacionales.}

En el análisis que sigue hemos considerado el estado $\left(n_{1}, n_{2}\right)=(1,3)$ porque en éste existen diferencias entre las distribuciones que provienen de las funciones simétrica y antisimétrica, por lo que si existe un efecto del confinamiento será más evidente. El estado $(1,1)$ no existe para la función antisimétrica por la presencia del hueco de Fermi, y en el estado $(2,2)$ las densidades de pares de las funciones simétrica y antisimétrica son iguales.

Los datos de la entropía de las marginales fueron obtenidos con Mathematica 6.0 [53], las entropías de pares fueron obtenidas usando una cuadratura de Gauss-Legendre de 12 puntos en cada subintervalo, trabajando con precisión doble, esquema probado con el cálculo de las normalizaciones. 


\subsubsection{Entropía de Shannon de las marginales.}

Recordamos aquí que las marginales de las funciones simétricas y antisimétricas son iguales como resultado de la ortonormalidad de las funciones de onda, por lo que estas densidades las etiquetaremos como indistinguibles, las marginales de la funciones no simétricas serán etiquetadas como distinguibles.

La figura (5.11) muestra el comportamiento de la suma entrópica de las marginales. En el caso de la densidad que pertenece a las partículas distinguibles este comportamiento (constante con respecto de $a$ ) es esperado porque las marginales son las densidades de una-partícula-en-una-caja y este

comportamiento ha sido mostrado analíticamente para ese caso [9]. Llama la atención que también se muestre un comportamiento constante en el caso de las partículas indistinguibles porque es un resultado numérico. Se puede argumentar, como en el caso de una-partícula-en-una-caja que este comportamiento es resultado del principio de incertidumbre y se implica que así es como la suma entrópica de las marginales contiene esta información (la deslocalización en el espacio de posición debido a que a crece, implica una localización en el espacio de momento).

Las figuras (5.12) y (5.13) muestran las entropías para los casos indistinguible y distinguible, respectivamente, en ambos espacios. Para valores pequeños de a la distribución de momentos es más deslocalizada que la de posiciones, comportamiento que se invierte para valores mayores de $a$, donde se localiza la distribución de momentos y se deslocaliza la de posi- 


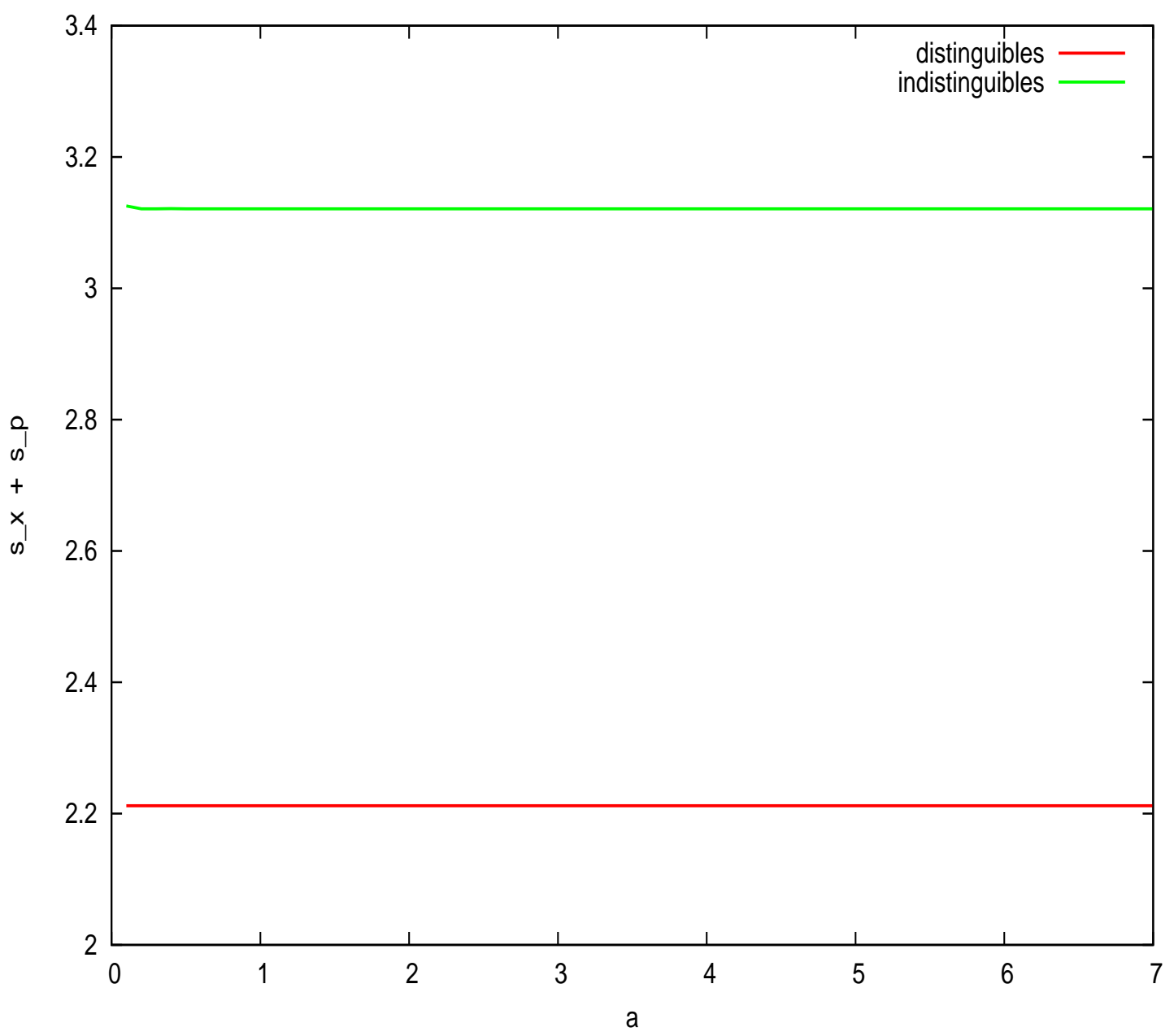

Figura 5.11: Suma entrópica de las marginales de la densidad de pares de partículas distinguibles (rojo) e indistinguibles (verde), como función de $a$, para $n_{1}=1$ y $n_{2}=3$. 


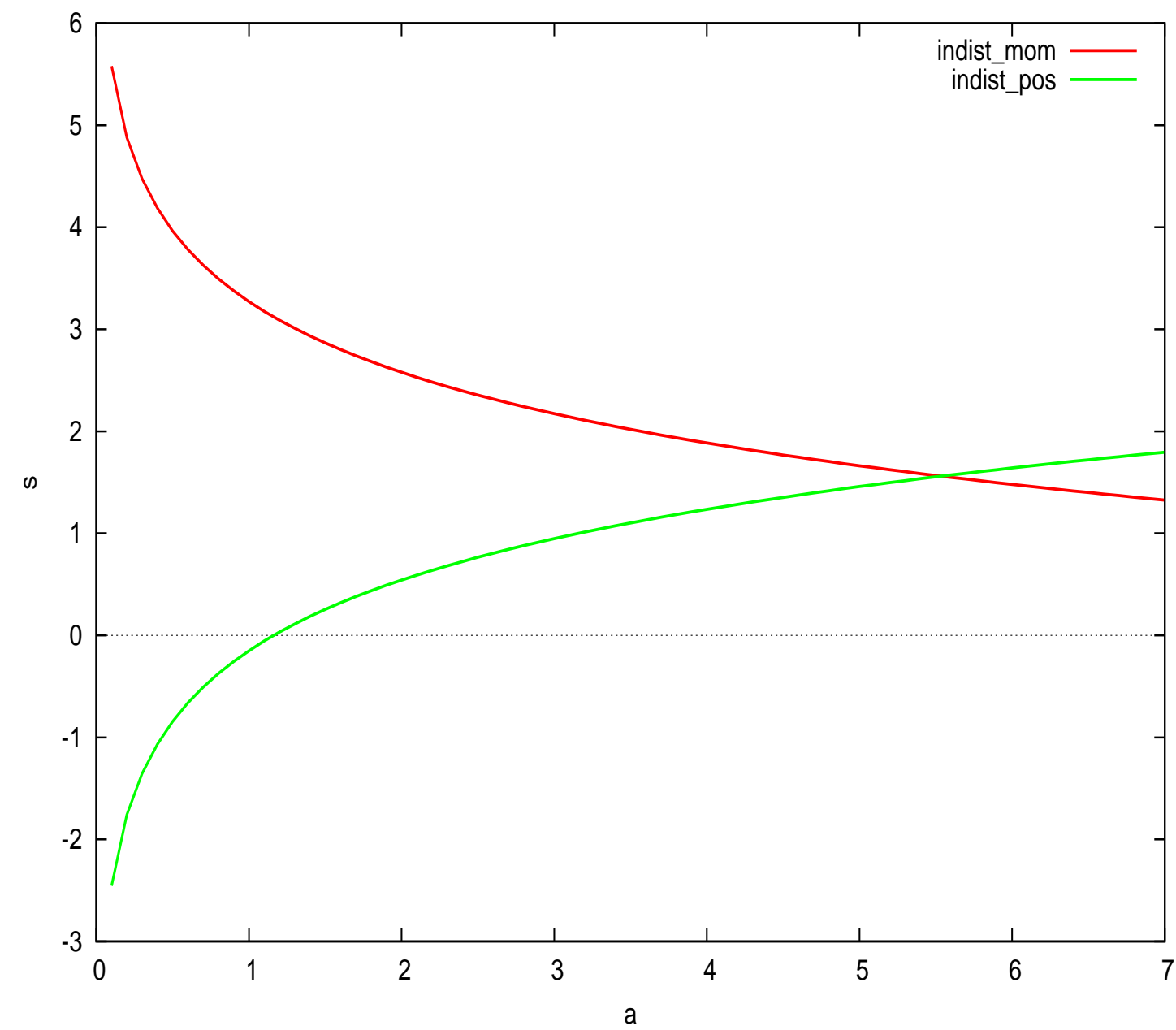

Figura 5.12: Entropía de Shannon en los espacios de posición (verde) y momento (rojo) de las marginales de la distribución para partículas indistinguibles, como función de $a$, para $n_{1}=1$ y $n_{2}=3$. 


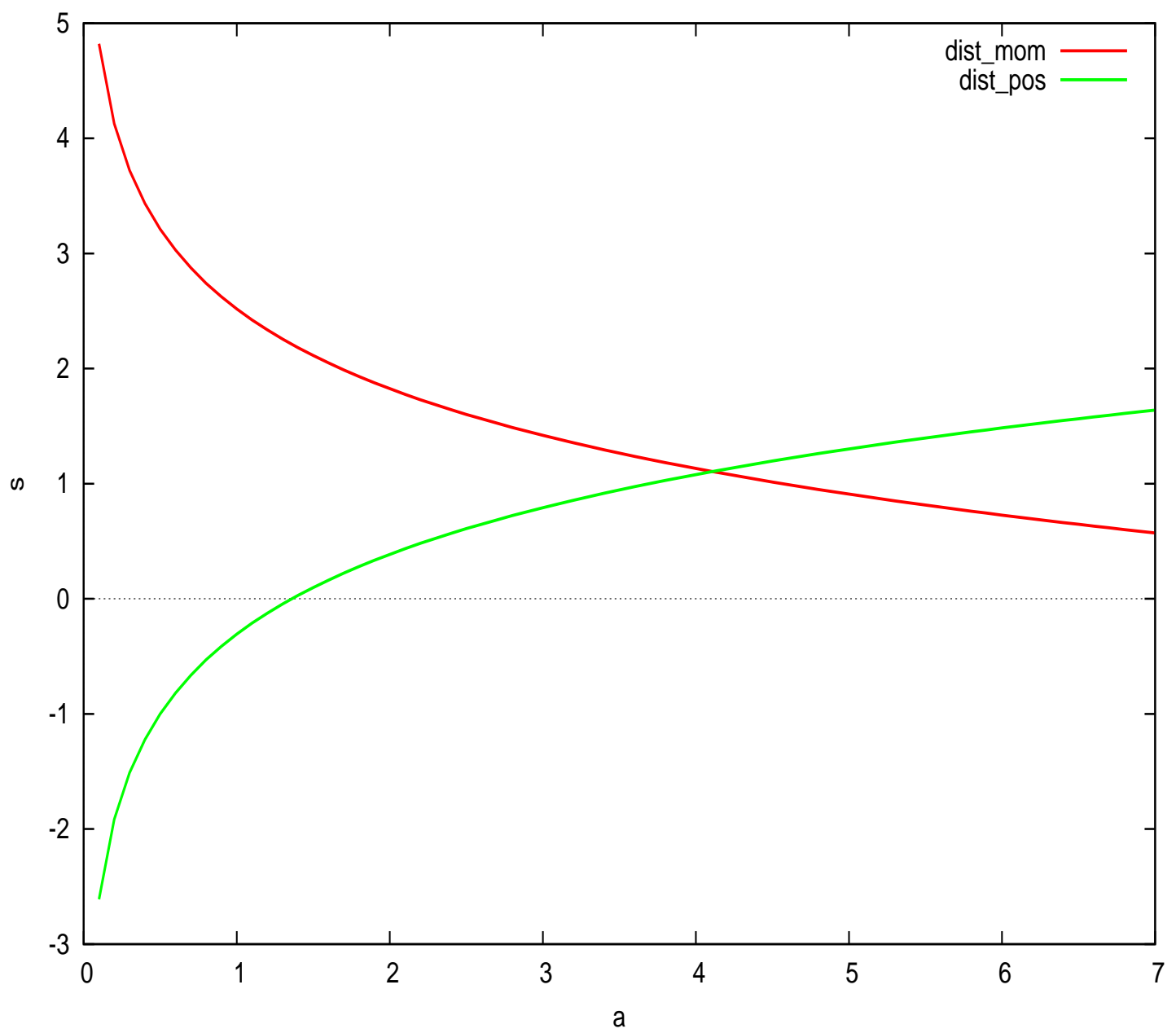

Figura 5.13: Entropía de Shannon en los espacios de posición (verde) y momento (rojo) de las marginales de la distribución para partículas distinguibles, como función de $a$, para $n_{1}=1$ y $n_{2}=3$. 
ciones. Esta transición se presenta en un valor mayor de $a$ para las partículas indistinguibles que para las distinguibles.

A través de la asociación del concepto "localización" con el concepto "condensación", puede argumentarse que antes del punto en que se cruzan las curvas $s_{\psi}$ y $s_{\widetilde{\psi}}$, existe una condensación mayor en el espacio de posición, después del punto la condensación mayor es en el espacio de momento. La energía de este sistema es inversamente proporcional al tamaño de la caja, para a mayores la energía (que es puramente cinética) es menor y por tanto las partículas se mueven más lentamente y la distribución de momentos es más localizada.

Puede preguntarse ¿qué sentido físico puede darse a la intersección de las rectas, el punto $s_{\psi}=s_{\widetilde{\psi}}$ ?, en un sentido entrópico este punto correspondería al estado del sistema que más se parecería a un estado clásico, porque las incertidumbres son iguales y por tanto el conocimiento de $x$ tiene el mismo efecto sobre el conocimiento de $p$ que el conocimiento de $p$ sobre $x$.

Las figuras (5.14) y (5.15) muestran las entropías en los espacios de posición y momento, respectivamente, comparando el comportamiento en cada espacio de partículas distinguibles e indistinguibles. En este estado se observa que en ambos espacios la distribución que proviene de la densidad de las partículas distinguibles se encuentra más localizada que la de partículas indistinguibles. La distancia entre las curvas se mantiene aproximadamente constante en todo el intervalo de $a$, coincidiendo las diferencias en hasta 6 y 3 cifras decimales, 


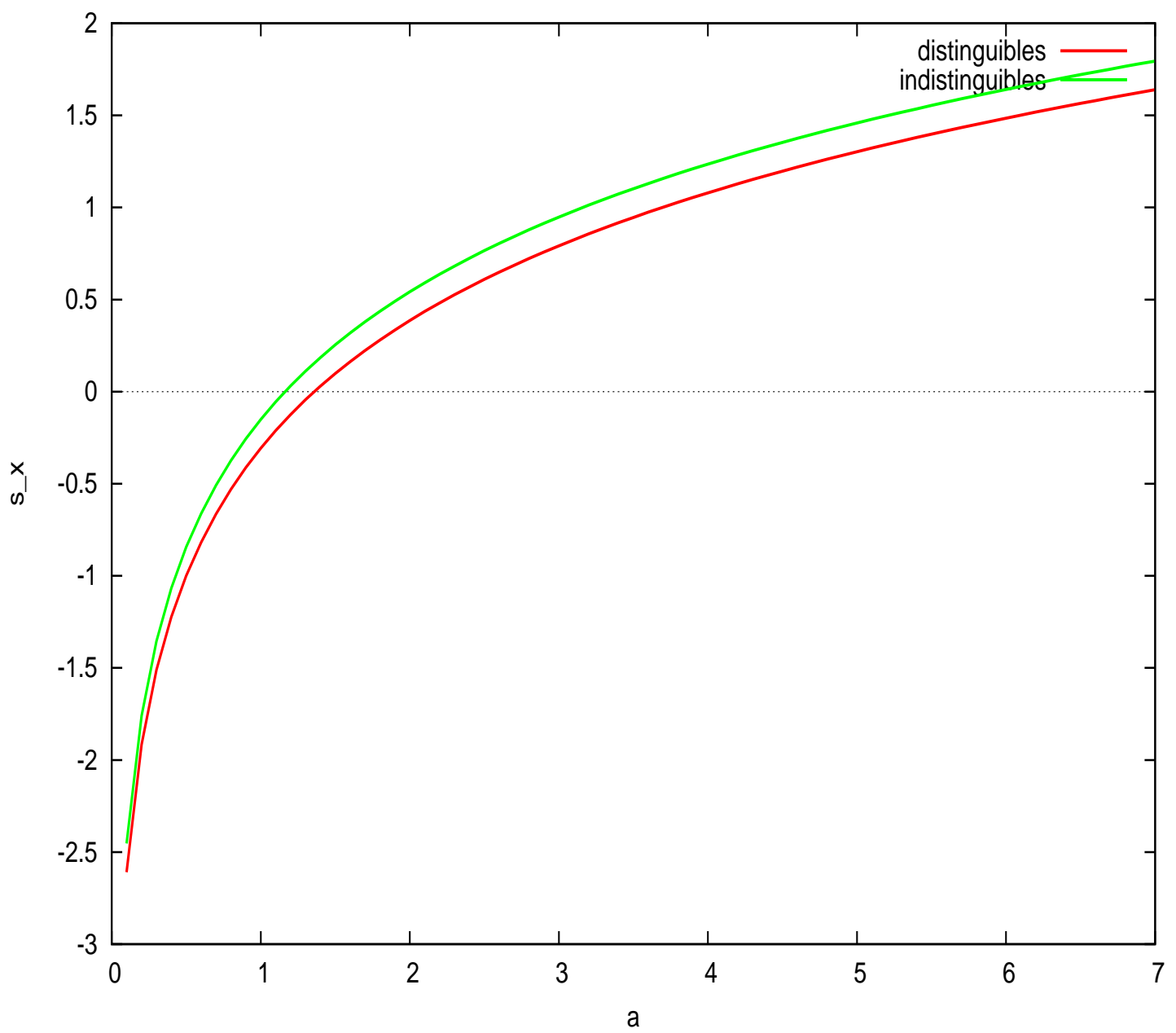

Figura 5.14: Entropía de Shannon en el espacio de posición de las marginales de las densidades de partículas distinguibles (rojo) e indistinguibles (verde), como función de $a$, para $n_{1}=1$ y $n_{2}=3$. 


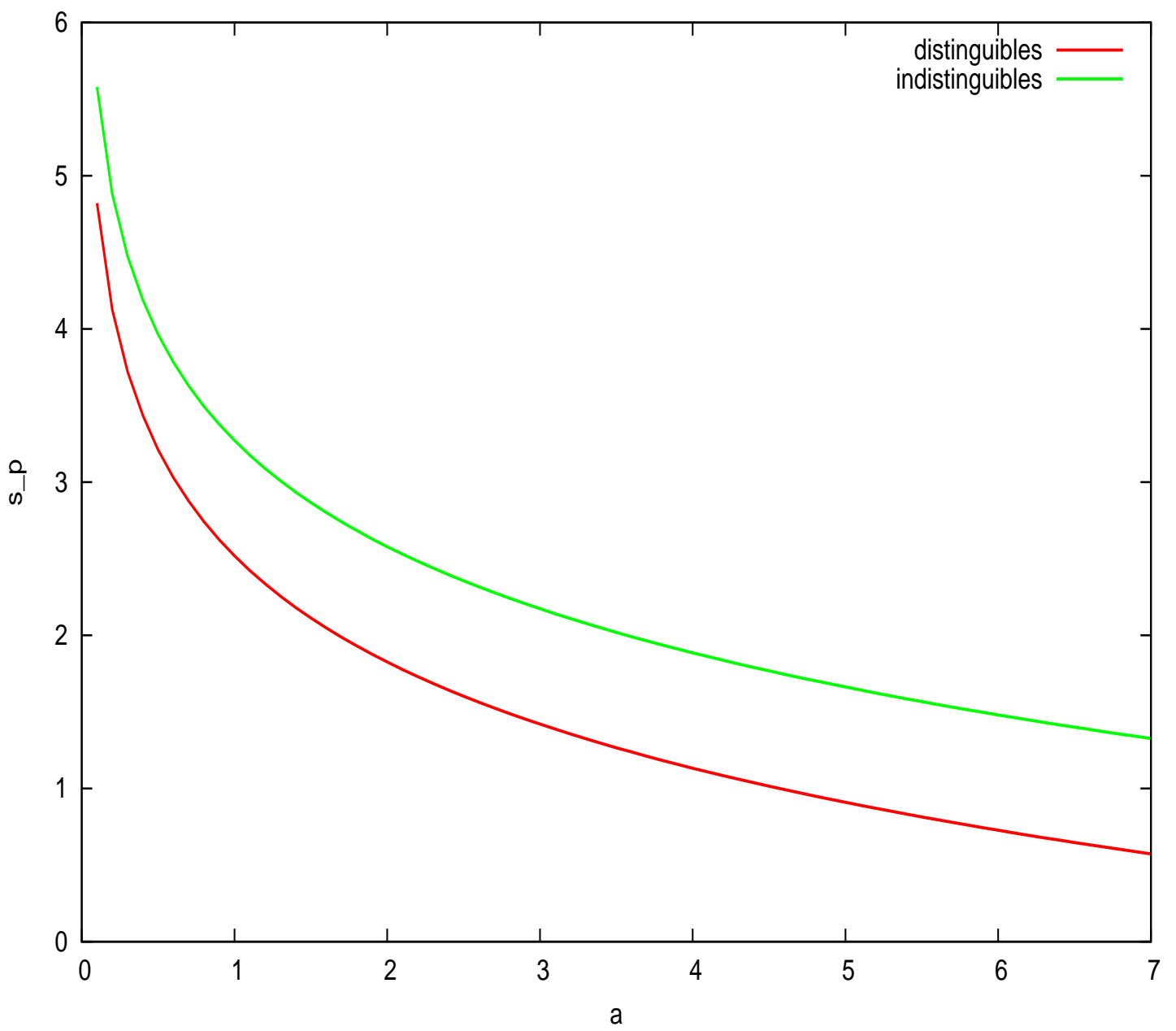

Figura 5.15: Entropía de Shannon en el espacio de momento de las marginales de las densidades de partículas distinguibles (rojo) e indistinguibles (verde), como función de $a$, para $n_{1}=1$ y $n_{2}=3$. 


\begin{tabular}{|c|c|}
\hline$s_{\psi_{\text {indist }}}-s_{\psi_{\text {dist }}}$ & 0.155973 \\
\hline$s_{\widetilde{\psi}_{\text {indist }}}-s_{\widetilde{\psi}_{\text {dist }}}$ & 0.753 \\
\hline
\end{tabular}

Debe notarse que las curvas son más cercanas en el espacio de posición que en el de momento, lo que sería indicativo de que en este último son más notorias las diferencias entre partículas distinguibles e indistinguibles que dependan de la localización de la distribución.

\subsubsection{Entropía de Shannon de la densidad de pares.}

La figura (5.16) muestra la suma entrópica para las diferentes simetrías de la función de onda. Hacemos notar el comportamiento constante de las curvas, pues además de ser un resultado numérico, la interpretación física está relacionada con el principio de incertidumbre al nivel de dos partículas, donde la deslocalización que se produce en el espacio de posición si aumentamos el tamaño de la caja, provoca una localización en el espacio de momento para que pueda mantenerse la suma constante.

Este comportamiento es esperado para la función no simétrica, pues la entropía de la densidad de pares es la suma de las entropías de una partículaen-una-caja, donde este comportamiento se ha mostrado analíticamente [9], en el caso de las funciones simetrizadas, este comportamiento no se puede inferir del resultado para una-partícula-en-una-caja, y permite argumentar que la simetría no influye en la forma en que está contenida la información sobre el principio de incertidumbre en el nivel de dos partículas.

Esta relación entre las entropías en cada espacio se muestra en las fig- 


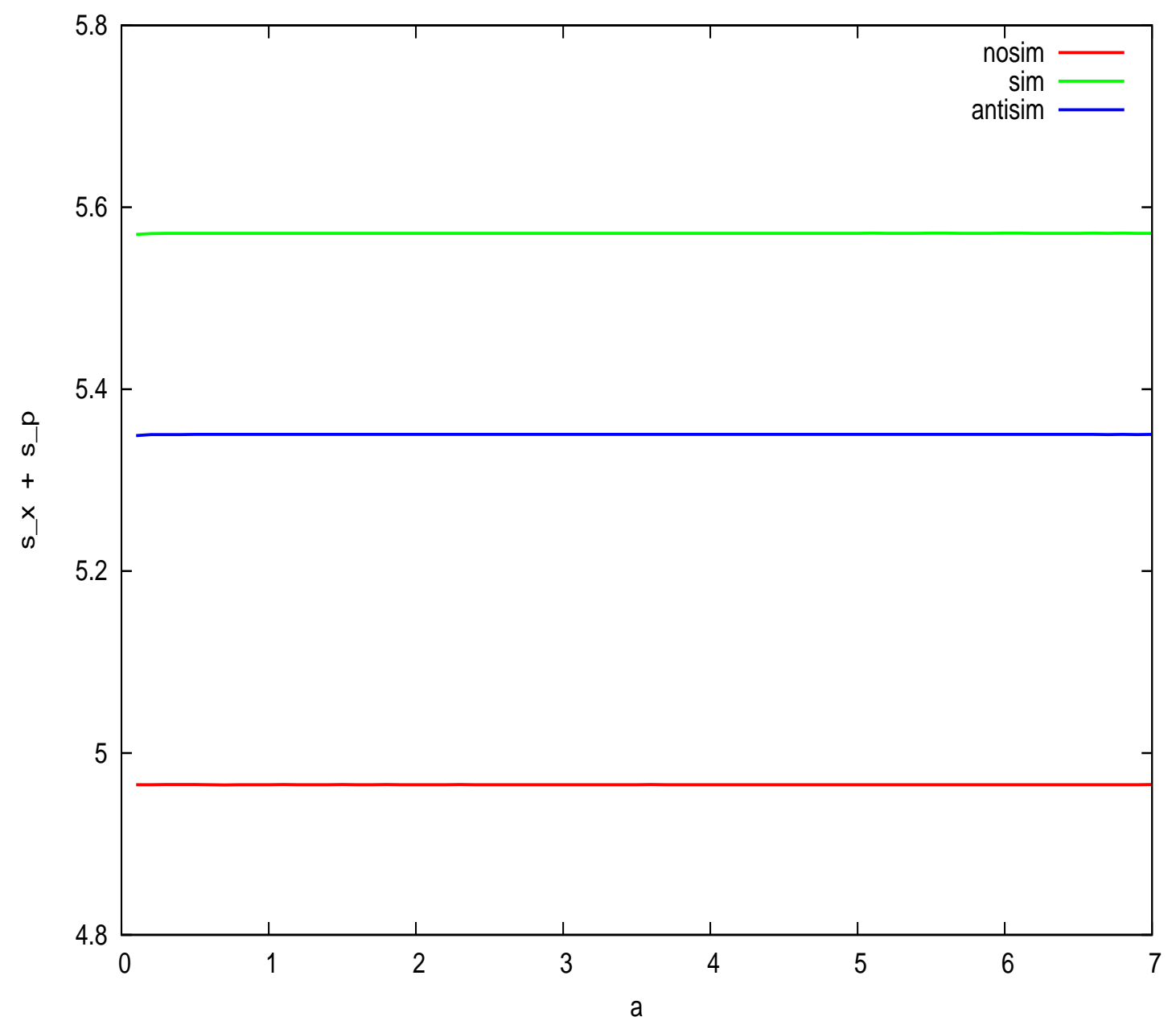

Figura 5.16: Suma entrópica de la densidad de pares de distribuciones no simétrica (rojo), simétrica (verde) y antisimétrica (azul), como función de $a$, para $n_{1}=1$ y $n_{2}=3$. 


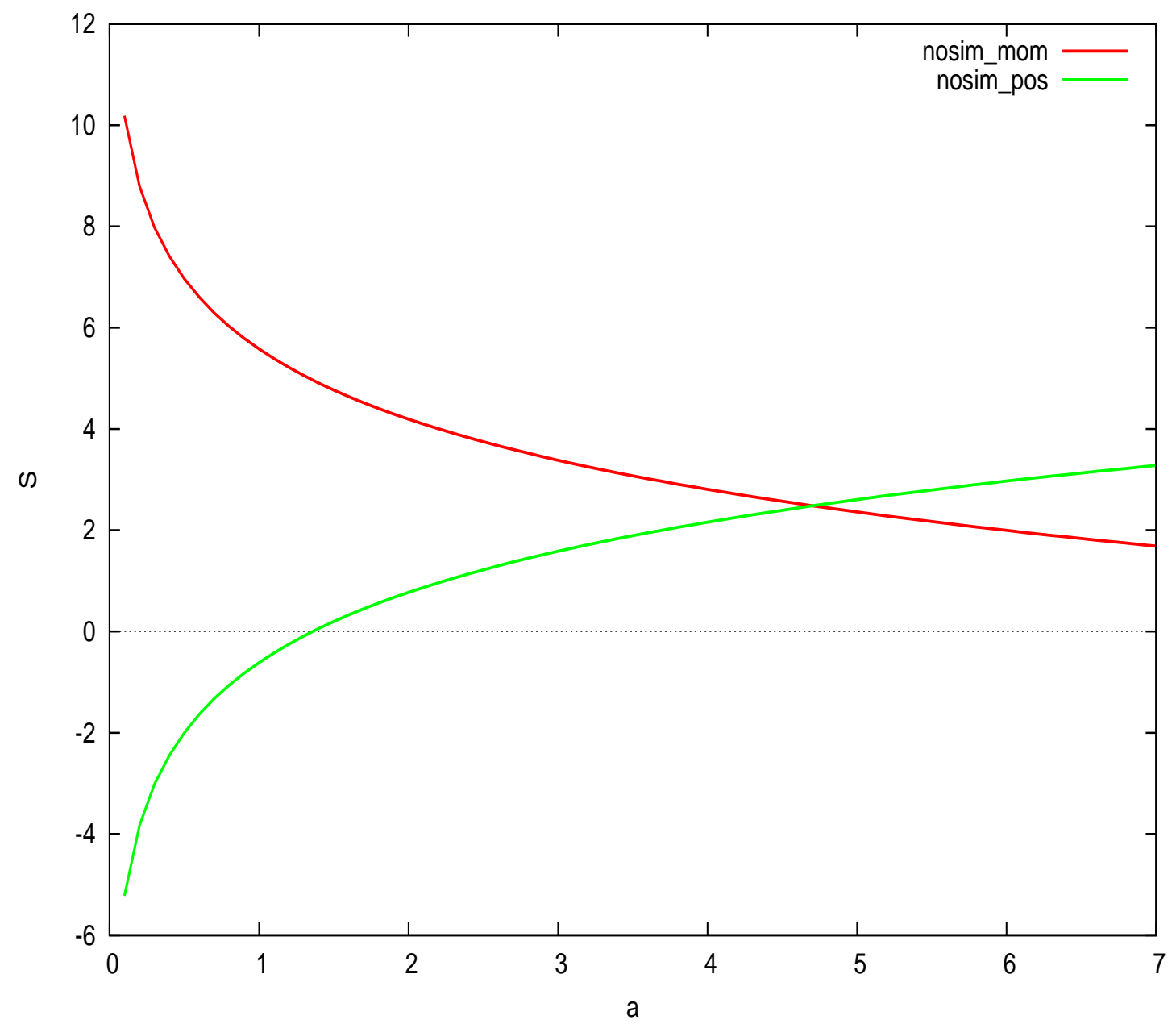

Figura 5.17: Entropía de Shannon de pares de la función no simétrica en los espacios de posición(verde) y momento (rojo), como función de $a$, para $n_{1}=1$ y $n_{2}=3$. 


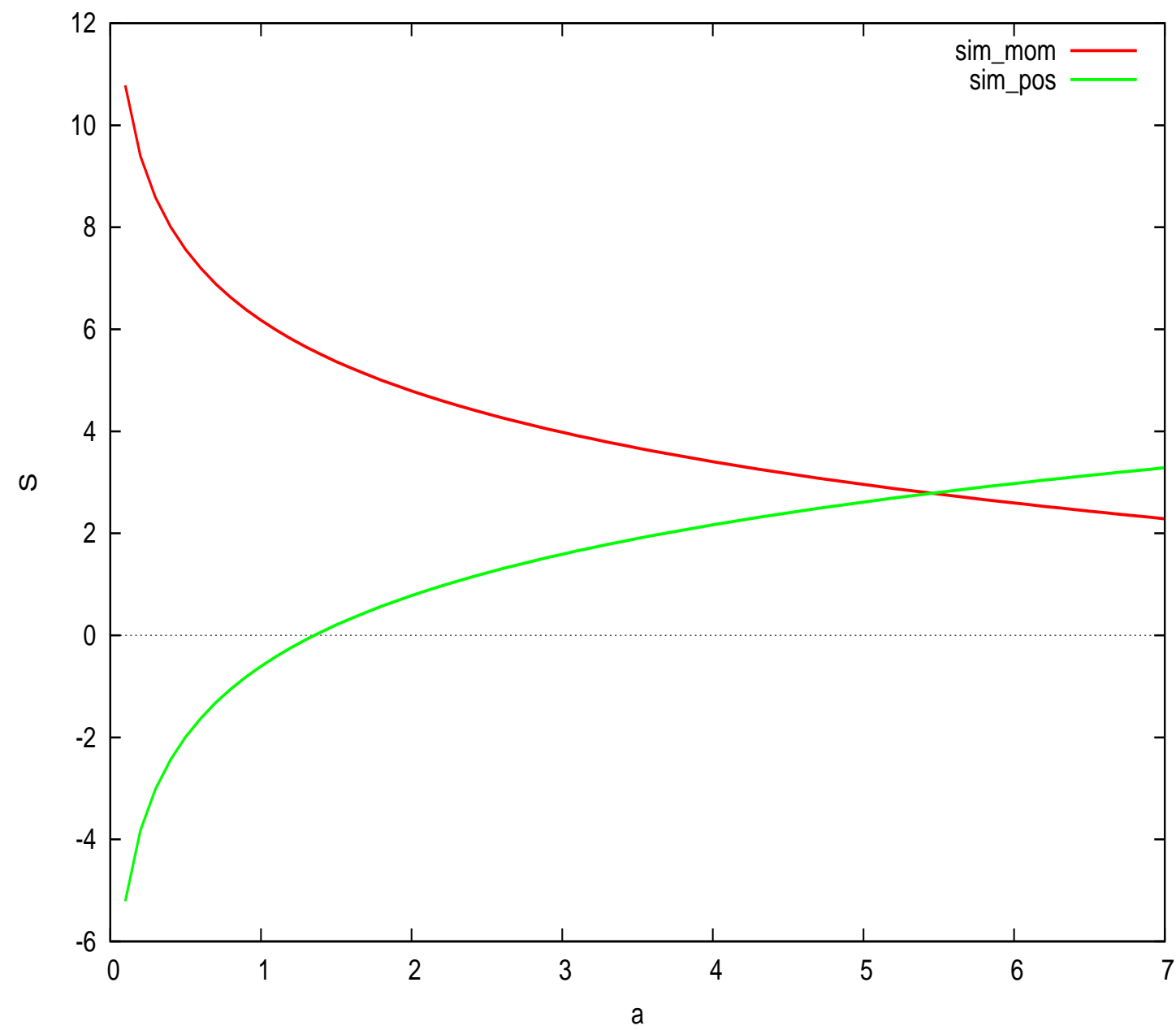

Figura 5.18: Entropía de Shannon de pares de la función simétrica en los espacios de posición(verde) y momento (rojo), como función de $a$, para $n_{1}=1$ y $n_{2}=3$. 


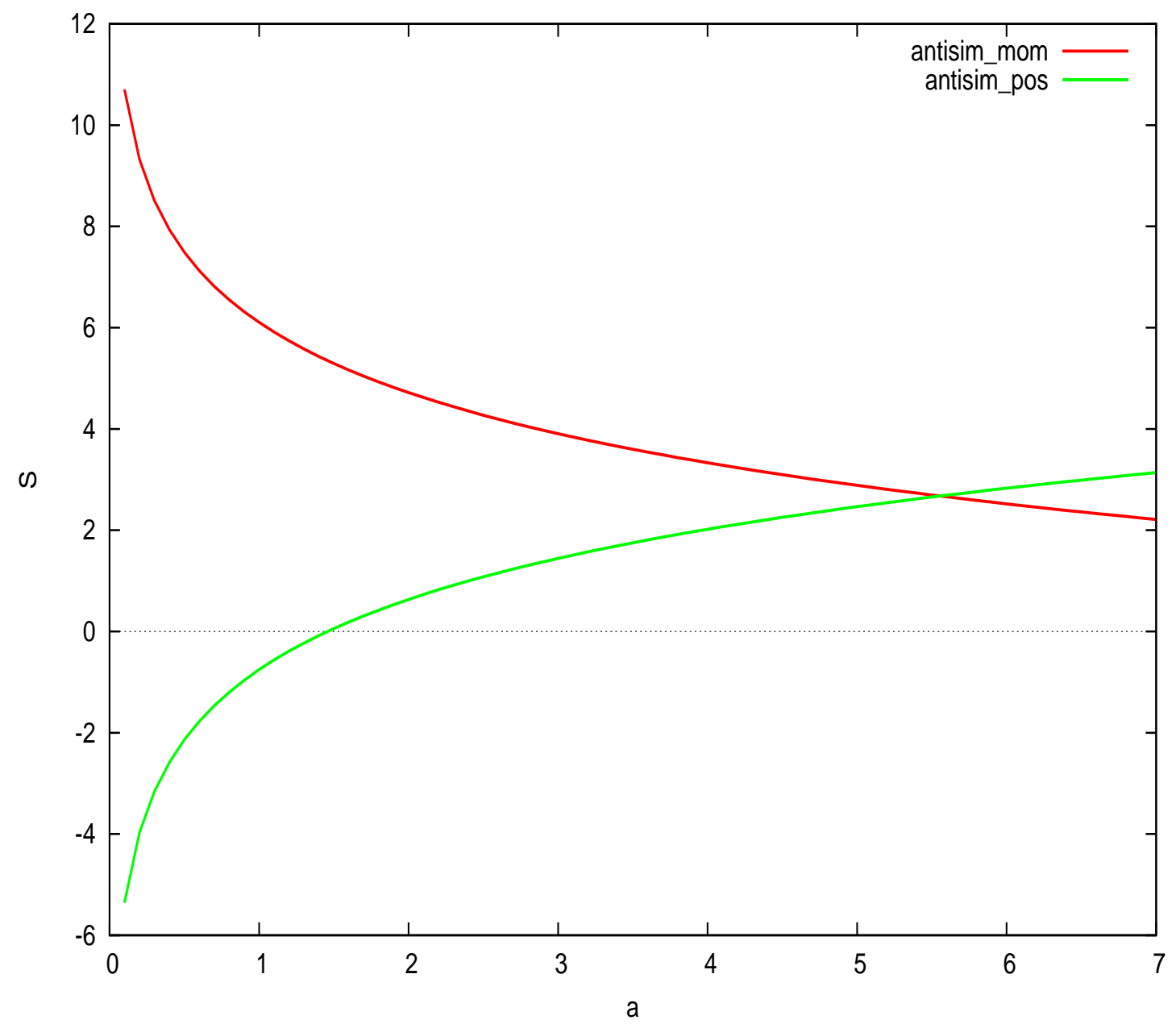

Figura 5.19: Entropía de Shannon de pares de la función antisimétrica en los espacios de posición(verde) y momento (rojo), como función de $a$, para $n_{1}=1$ y $n_{2}=3$. 
uras (5.17), (5.18) y (5.19) para las funciones no simétrica, simétrica y antisimétrica, respectivamente.

De manera similar al caso de las marginales, puede observarse que el punto de la transición en que la densidad de posiciones deja de ser más localizada que la de momentos y el comportamiento se invierte, ocurre para una a mayor en el caso de las funciones simetrizadas (correspondientes a partículas indistinguibles). Cabe anotar también que entre las dos simetrizadas el punto de cruce es ligeramente menor para la función simétrica (donde ocurre en $5.4<a<5.5$ ) que para la antisimétrica (donde ocurre en $5.5<a<5.6$ ), como se observa,

\begin{tabular}{|c|c|c|c|c|}
\hline$a$ & $s_{\boldsymbol{\Psi}_{\text {sim }}}$ & $s_{\widetilde{\boldsymbol{\Psi}}_{\text {sim }}}$ & $s_{\boldsymbol{\Psi}_{\text {antisim }}}$ & $s_{\widetilde{\boldsymbol{\Psi}}_{\text {antisim }}}$ \\
\hline 5.4 & 2.7662 & 2.8052 & 2.6189 & 2.7313 \\
\hline 5.5 & 2.8029 & 2.7685 & 2.6556 & 2.6946 \\
\hline 5.6 & 2.8389 & 2.7324 & 2.6916 & 2.6585 \\
\hline
\end{tabular}

Las figuras (5.20) y (5.21) muestran las entropías de las diferentes simetrías en los espacios de posición y momento, respectivamente. La distribución que proviene de la función simétrica es la más deslocalizada en ambos espacios. En este respecto, la tendencia se invierte para las funciones no simétrica y antisimétrica, la primera más deslocalizada que la segunda en el espacio de posición, pero más localizada en el de momento. Este comportamiento y el hecho de que la distancia entre las curvas es aproximadamente constante hasta las cifras mostradas, se ilustra con la tabla siguiente, 


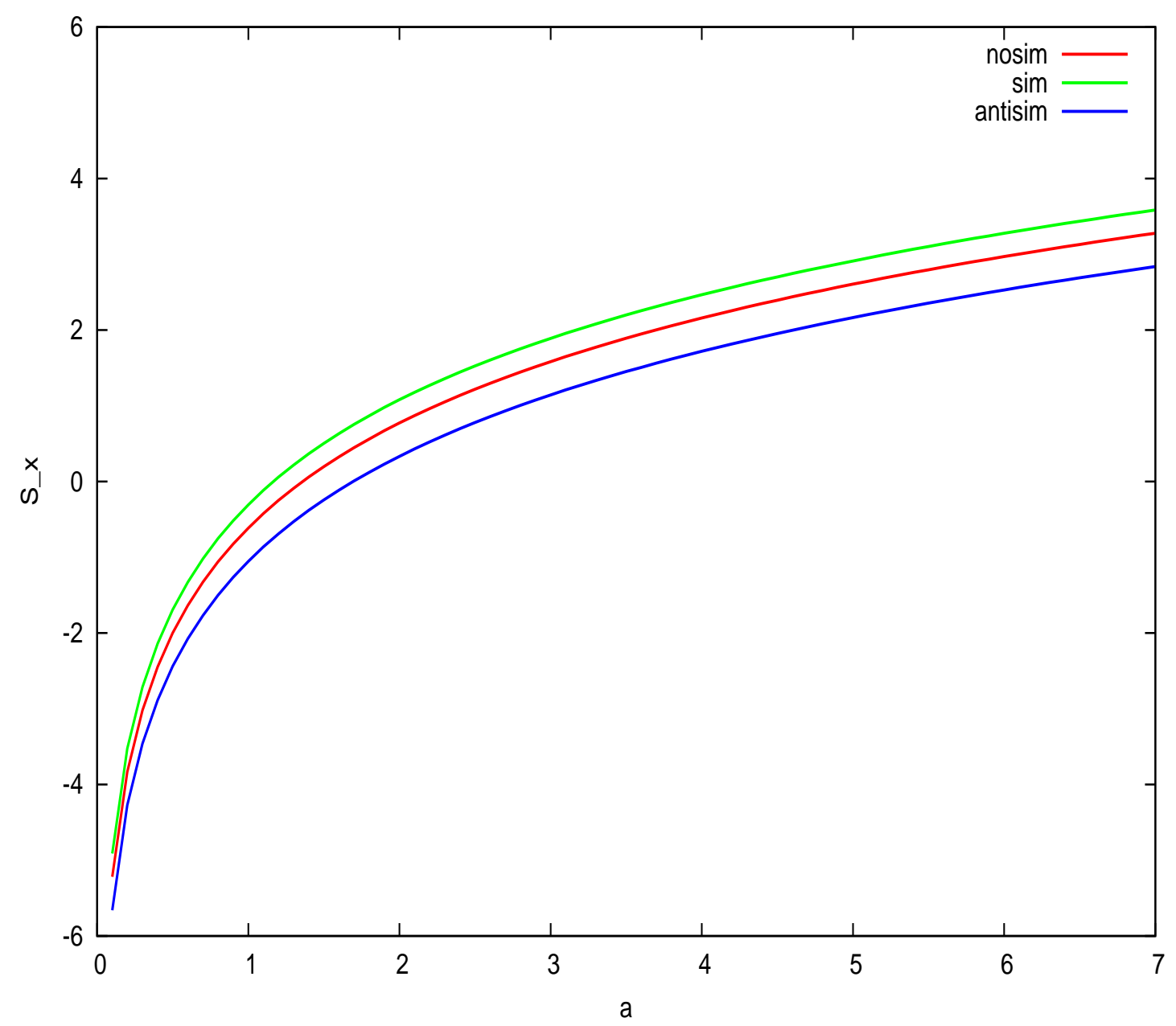

Figura 5.20: Entropía de Shannon de pares de las funciones no simétrica (rojo), simétrica (verde) y antisimétrica (azul) en el espacio de posición, como función de $a$, para $n_{1}=1$ y $n_{2}=3$. A la curva correspondiente a la función simétrica se le ha sumado 0.3 unidades y a la correspondiente a la antisimétrica se le ha restado 0.3 unidades para dar mayor claridad a la gráfica sin alterar sus características fundamentales. 


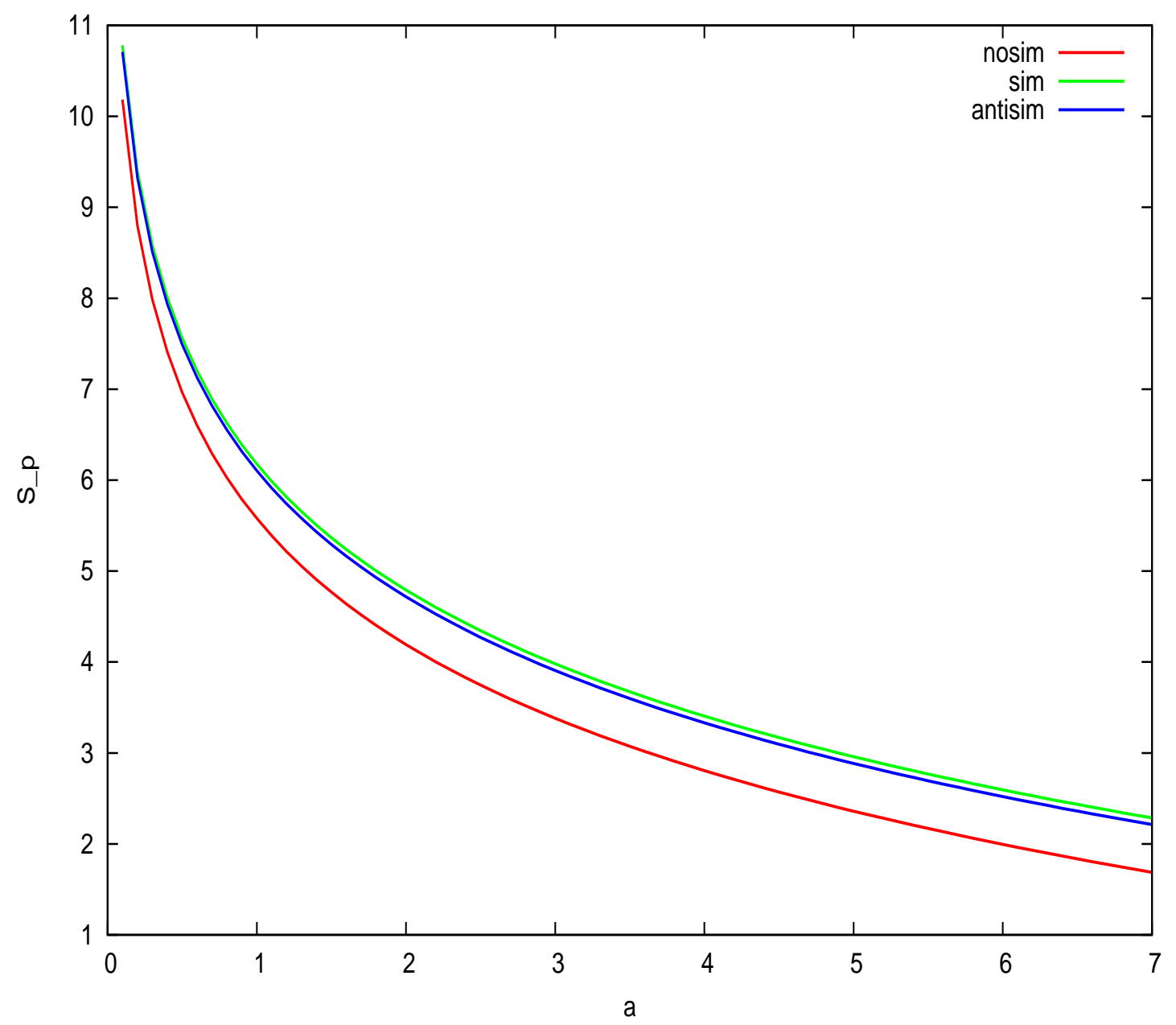

Figura 5.21: Entropía de Shannon de pares de las funciones no simétrica (rojo), simétrica (verde) y antisimétrica (azul) en el espacio de momento, como función de $a$, para $n_{1}=1$ y $n_{2}=3$. 


\begin{tabular}{|c|c|}
\hline$s_{\boldsymbol{\Psi}_{\text {sim }}}-s_{\mathbf{\Psi}_{\text {nosim }}}$ & $7.13785\left(10^{-3}\right)$ \\
\hline$s_{\widetilde{\boldsymbol{\Psi}}_{\text {sim }}}-s_{\widetilde{\boldsymbol{\Psi}}_{\text {nosim }}}$ & 0.599 \\
\hline \hline$s_{\boldsymbol{\Psi}_{\text {sim }}}-s_{\boldsymbol{\Psi}_{\text {antisim }}}$ & 0.147324 \\
\hline$s_{\widetilde{\boldsymbol{\Psi}}_{\text {sim }}}-s_{\widetilde{\boldsymbol{\Psi}}_{\text {antisim }}}$ & 0.074 \\
\hline \hline$s_{\boldsymbol{\Psi}_{\text {nosim }}}-s_{\boldsymbol{\Psi}_{\text {antisim }}}$ & 0.140186 \\
\hline$s_{\widetilde{\boldsymbol{\Psi}}_{\text {nosim }}}-s_{\widetilde{\boldsymbol{\Psi}}_{\text {antisim }}}$ & -0.525 \\
\hline
\end{tabular}

Las diferencias mostradas son consistentes con la información proveniente de las marginales, en cuanto a que las diferencias entre las partículas distinguibles e indistinguibles que dependan en la localización de la distribución serían más evidentes en el espacio de momento, porque ahí son más distantes las entropías de ambas. En cambio, la distinción entre las partículas que obedezcan funciones simétricas y aquellas que obedezcan funciones antisimétricas, atendiendo a la localización de la distribución, sería más evidente en el espacio de posición.

El cambio de signo de la diferencia en el espacio de momento de las entropías de no simétrica y antisimétrica hace claro que es la única tendencia que se invierte en las curvas graficadas.

\subsubsection{Información mutua.}

Las figuras (5.22) y (5.23) muestran que la correlación que predice la información mutua no cambia con el tamaño de la caja ( $I$ es constante). La curva correspondiente a $I_{p}$ comienza en 1 porque antes de este valor se presentan algunas fluctuaciones que pudieran provenir de inestabilidades 


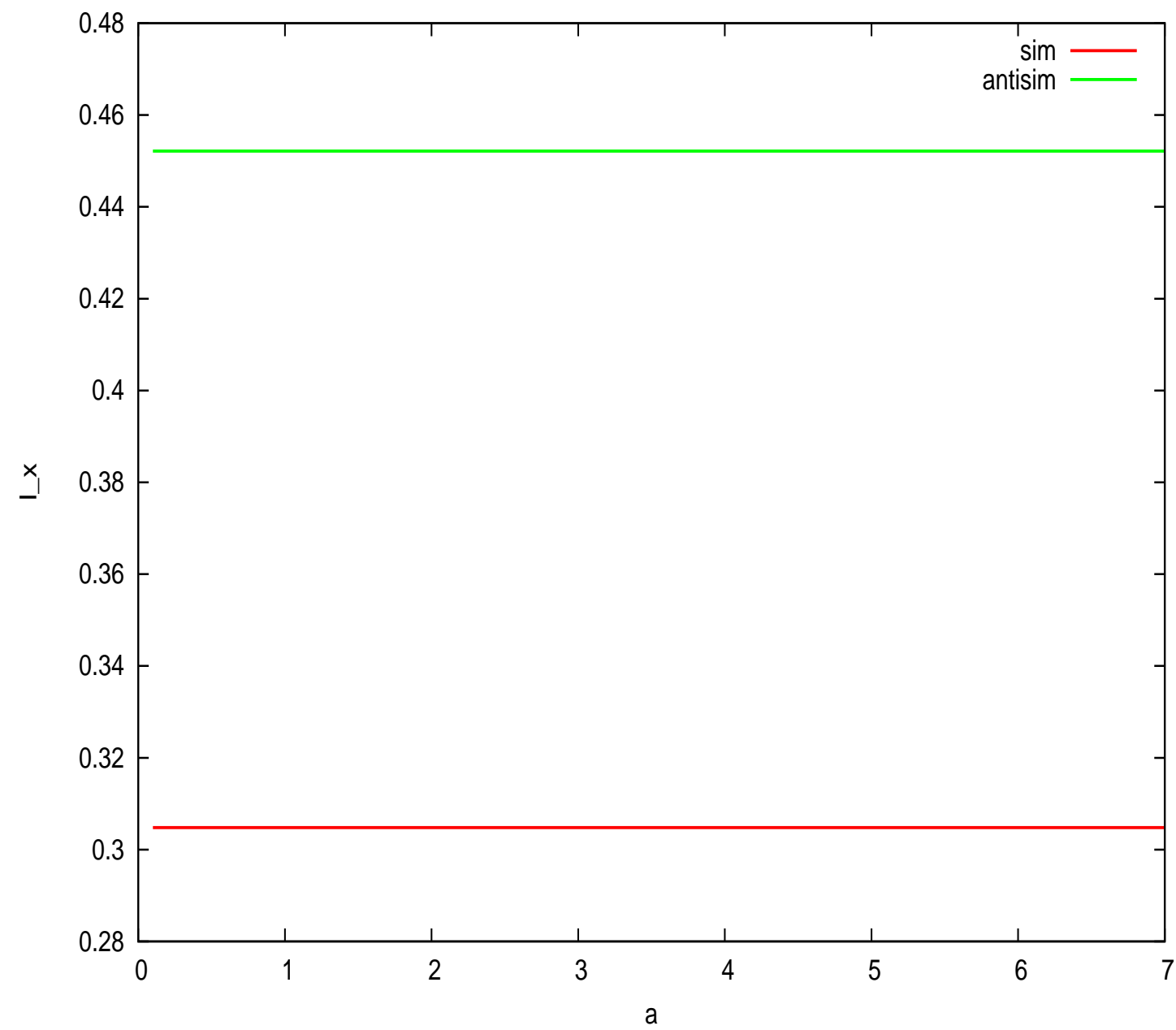

Figura 5.22: Información mutua de las funciones simétrica (rojo) y antisimétrica (verde) en el espacio de posición, como función de $a$, para $n_{1}=1$ y $n_{2}=3$. 


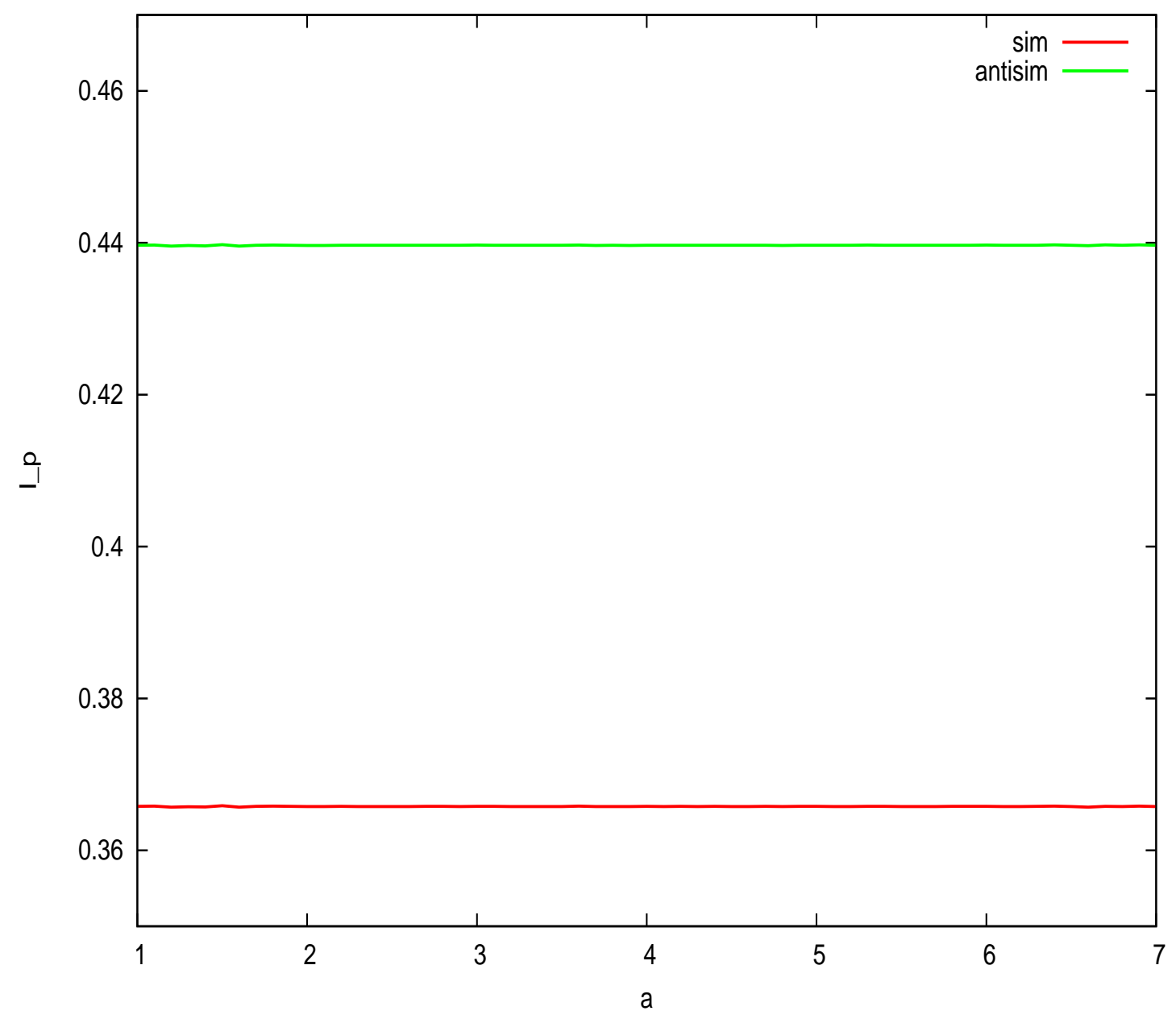

Figura 5.23: Información mutua de las funciones simétrica (rojo) y antisimétrica (verde) en el espacio de momento, como función de $a$, para $n_{1}=1$ y $n_{2}=3$. 
numéricas. La correlación para la función simétrica es menor que para la función antisimétrica en ambos espacios.

En el caso de la función simétrica, la correlación es mayor en el espacio de momento que en el de posición. En el caso de la función antisimétrica, la correlación es mayor en el espacio de posición.

\subsection{Correlación posición-momento.}

En el caso de dos partículas, la correlación posición-momento tiene cuatro componentes, $\left(x_{1}, p_{1}\right),\left(x_{2}, p_{2}\right),\left(x_{1}, p_{2}\right)$ y $\left(x_{2}, p_{1}\right)$. Las primeras dos son similares a la correlación presente en una-partícula-en-una-caja, pues los operadores asociados a cada partícula no conmutan y una parte de la correlación proviene de este hecho.

Las correlaciones $\left(x_{1}, p_{2}\right)$ y $\left(x_{2}, p_{1}\right)$ se dan entre operadores que si conmutan y son las correlaciones detrás del argumento de Einstein-Podolsky-Rosen (EPR) [54], no existe en este caso una correlación análoga en la mecánica clásica, puede entonces ser etiquetada como "correlación cuántica".

Existen cuatro informaciones mutuas de orden mayor que pueden definirse,

$$
\begin{array}{r}
I_{a}=\int d x_{1} d x_{2} d p_{1} d p_{2} W\left(x_{1}, p_{1}, x_{2}, p_{2}\right) \ln \left[\frac{W\left(x_{1}, p_{1}, x_{2}, p_{2}\right)}{\left|\boldsymbol{\Psi}\left(x_{1}, x_{2}\right)\right|^{2}\left|\widetilde{\boldsymbol{\Psi}}\left(p_{1}, p_{2}\right)\right|^{2}}\right] \\
=s_{\boldsymbol{\Psi}}+s_{\widetilde{\boldsymbol{\Psi}}}-s_{W}=s_{T}-s_{W},
\end{array}
$$




$$
\begin{aligned}
& I_{b}=\int d x_{1} d x_{2} d p_{1} d p_{2} W\left(x_{1}, p_{1}, x_{2}, p_{2}\right) \ln \left[\frac{W\left(x_{1}, p_{1}, x_{2}, p_{2}\right)}{\left|\psi\left(x_{1}\right)\right|^{2}\left|\psi\left(x_{2}\right)\right|^{2}\left|\widetilde{\psi}\left(p_{1}\right)\right|^{2}\left|\widetilde{\psi}\left(p_{2}\right)\right|^{2}}\right] \\
& =2 s_{\psi}+2 s_{\widetilde{\psi}}-s_{W}=2 s_{t}-s_{W} \text {, }
\end{aligned}
$$

$$
\begin{aligned}
& I_{c}=\int d x_{1} d x_{2} d p_{1} d p_{2} W\left(x_{1}, p_{1}, x_{2}, p_{2}\right) \ln \left[\frac{W\left(x_{1}, p_{1}, x_{2}, p_{2}\right)}{W\left(x_{1}, p_{1}\right) W\left(x_{2}, p_{2}\right)}\right] \\
& =2 s_{w}-s_{W}
\end{aligned}
$$

e

$$
\begin{aligned}
I_{d}=\int d x_{1} d x_{2} d p_{1} d p_{2} W\left(x_{1}, p_{1}, x_{2}, p_{2}\right) \ln & {\left[\frac{W\left(x_{1}, p_{1}, x_{2}, p_{2}\right)}{\rho\left(x_{1}, p_{2}\right) \rho\left(x_{2}, p_{1}\right)}\right] } \\
& =s_{\rho_{1,2}}+s_{\rho_{2,1}}-s_{W} .
\end{aligned}
$$

\subsubsection{Relación entre la correlación posición-momento y la proveniente de la indistinguibilidad.}

La información mutua es una medida de qué tan separada está una distribución dada de otra que constituye una referencia (el producto de sus marginales), en términos de sus incertidumbres.

En $I_{a}$ la referencia es el producto de las distribuciones de pares en los espacios de posición y momento (marginales de la función de Wigner), que 
contienen los efectos de la simetría de la función de onda (en su caso también de coulomb), por tanto ésta contendría exclusivamente las correlaciones posición-momento al nivel de dos partículas.

$I_{b}$ tiene como referencia el producto de las distribuciones de una partícula, marginales de las distribuciones de dos partículas, ésta contendría entonces toda la correlación presente en el sistema. La diferencia entre estas dos informaciones mutuas contendría las correlaciones provenientes de la indistinguibilidad presentes en el sistema,

$$
I_{b}-I_{a}=2 s_{t}-s_{W}-\left(s_{T}-s_{W}\right)=I_{x}+I_{p}
$$

\subsubsection{Relación entre el entanglement cuántico y la in- distinguibilidad.}

$I_{c}$ tiene como referencia las funciones de Wigner de una partícula, también marginales de la función de dos partículas, y mide la no separabilidad de la segunda en términos de funciones de una sola partícula, contendría la correlación total de una partícula sobre otra.

$I_{d}$ tiene como referencia las distribuciones que sugieren la existencia de correlación estadística $\left(x_{1}, p_{2}\right)$ y $\left(x_{2}, p_{1}\right)$, si tomamos la diferencia obtenemos,

$$
I_{c}-I_{d}=\int d x_{1} d x_{2} d p_{1} d p_{2} W\left(x_{1}, p_{1}, x_{2}, p_{2}\right) \ln \left[\frac{W\left(x_{1}, p_{1}\right) W\left(x_{2}, p_{2}\right)}{\rho\left(x_{1}, p_{2}\right) \rho\left(x_{2}, p_{1}\right)}\right] .
$$

Esta diferencia puede pensarse también en términos de la indistinguibilidad, puesto que si $x_{1}$ es sustituible libremente por $x_{2}$ o $p_{1}$ por $p_{2}$ no existe 
diferencia entre las distribuciones y la existencia de correlaciones entre las partículas $\left(I_{c}\right)$ sería un indicativo suficiente de la presencia del entanglement cuántico.

Es parte de las perspectivas de este trabajo construir la función de Wigner para dos partículas en una caja y con ésta estudiar las ecuaciones planteadas en este apartado. 


\section{Resumen de los resultados}

\section{UNA-PARTÍCULA-EN-UNA-CAJA.}

- En este modelo es mejor la formulación entrópica del principio de incertidumbre que la formulación convencional en términos de las varianzas.

- $\left(s_{\psi}+s_{\widetilde{\psi}}\right)$ y $s_{w}$ son constantes con $a$, el tamaño de la caja.

- $\operatorname{Cov}(\hat{x}, \hat{p})=0$, mientras que la información mutua es capaz de detectar la correlación posición-momento en el sistema de una partícula en la caja.

- El comportamiento de las diferentes definiciones posibles de la información mutua son consistentes con la interpretación de que la correlación posición-momento es mayor en la mecánica clásica que en la cuántica. Según una formulación del principio de correspondencia, la mecánica cuántica debe reducir a la clásica para valores muy grandes de $n$, así, esperaríamos que la correlación creciera con $n$ y efectivamente ocurre. 
- El hecho de que $I=\left|s_{\psi}\right|+\left|s_{\widetilde{\psi}}\right|-\left|s_{w}\right|=s_{\psi}-s(x \mid p)=s_{\widetilde{\psi}}-s(p \mid x)$ sea negativa, puede interpretarse en el sentido de que "el conocimiento de una variable hace aumentar la incertidumbre en la variable conjugada".

\section{DOS-PARTÍCULAS-EN-UNA-CAJA.}

Nivel de una partícula (marginales).

- $\left(s_{\psi}+s_{\widetilde{\psi}}\right)$ de las marginales de las funciones es constante con $a$, resalta el caso de las partículas indistinguibles porque es un resultado numérico.

- En este nivel la indistinguibilidad implica mayor deslocalización en ambos espacios.

- El punto de cruce $s_{\psi}=s_{\widetilde{\psi}}$ ocurre a mayores valores de $a$ para partículas indistinguibles.

- En cuanto a su estructura, las distribuciones de partículas distinguibles se diferencian más de las de indistinguibles en el espacio de momento. 
Nivel de dos partículas.

- $\left(s_{\boldsymbol{\Psi}}+s_{\widetilde{\boldsymbol{\Psi}}}\right)$ es constante con $a$, lo que indica un principio de incertidumbre al nivel de dos partículas y que la simetría no influye en la manera en que está contenida esta información en las distribuciones.

- En este nivel, la indistinguibilidad implica mayor deslocalización de las distribuciones en el espacio de momento, en tanto que implica mayor localización de las mismas en el espacio de posición.

- En general, las distribuciones que provienen de las funciones antisimétricas están más localizadas que las que provienen de funciones simétricas en ambos espacios.

- El punto de cruce $s_{\boldsymbol{\Psi}}=s_{\widetilde{\boldsymbol{\Psi}}}$, ocurre en valores menores de $a$ para partículas distinguibles que para partículas indistinguibles. En valores menores de a la distribución de posición está mas localizada (condensada) que la de momento, para valores mayores de $a$ ocurre lo contrario.

- El punto de cruce $s_{\Psi}=s_{\widetilde{\boldsymbol{\Psi}}}$, ocurre en valores menores de $a$ para partículas que obedecen funciones simétricas que para las que obedecen funciones antisimétricas. En valores menores de $a$ la distribución de posición está más localizada (condensada) que la de momento, para valores mayores de $a$ ocurre lo contrario. 
- Las diferencias en la estructura de las distribuciones de partículas distinguibles e indistinguibles son mayores en el espacio de momento.

- Las diferencias en la estructura de las distribuciones que provienen de las funciones simétrica y antisimétrica son mayores en el espacio de posición.

Correlación.

- En general, existe más correlación en las distribuciones que provienen de funciones antisimétricas que en las que provienen de funciones simétricas en ambos espacios.

- En la mayoría de los estados graficados la correlación es mayor en el espacio de momento que en el de posición para las distribuciones que provienen de funciones antisimétrica y simétrica.

- La correlación en el espacio de posición en las funciones simétrica y antisimétrica es mayor en estados de baja energía que en los de mayor energía.

- La correlación crece con la energía en el espacio de momento. 


\section{Conclusiones generales}

La entropía de Shannon de la función de Wigner contiene la información sobre el principio de incertidumbre en la misma manera que la suma entrópica para este sistema, donde se observa que la deslocalización en $x$ implica localización en $p$, y viceversa, independientemente de $a$, el tamaño de la caja.

El empleo de la entropía de Shannon de la función de Wigner permite detectar correlación entre $x$ y $p$ en el modelo de una partícula en la caja, a diferencia de la covarianza cuántica y las funciones de correlación de orden mayor, que son cero no porque no exista correlación, porque al menos una parte de ésta es evidente en el principio de incertidumbre. La interpretación de que la covarianza es cero es que por la simetría del problema en cada punto de la caja existe la misma probabilidad de momento positivo o negativo, por lo que $<\hat{p}>=<\hat{x} \hat{p}>=0$.

Las diferentes definiciones posibles de la información mutua para el estudio de la correlación posición-momento tienen implicaciones interesantes para la teoría pues su interpretación puede relacionarse con algunas características del formalismo cuántico (el conocimiento de una variable implica 
aumentar la incertidumbre de la variable conjugada), o con la relación entre la correlación $(x, p)$ presente en los sistemas cuánticos y los clásicos (mayor en estos últimos).

Discutiremos ahora las distribuciones de dos partículas y sus marginales.

La indistinguibilidad implica mayor deslocalización en ambos espacios en el nivel de una partícula (en las marginales), mientras que en el nivel de dos partículas la indistinguibilidad implica mayor deslocalización en el espacio de momento, pero mayor localización en el de posición.

La información que proviene del nivel de una partícula es diferente de la que proviene del nivel de dos partículas, por lo que es relevante el estudio de las entropías de pares porque puede arrojar información no visible en las distribuciones reducidas de una partícula.

En el nivel de una partícula, la suma entrópica de las partículas indistinguibles es contante con $a$. El punto $s_{\psi}=s_{\widetilde{\psi}}$ ocurre en un valor mayor de $a$ para partículas indistinguibles que para distinguibles. En valores pequeños de a la posición está más localizada (condensada) que el momento, y en valores mayores de $a$ el comportamiento se invierte.

En este nivel (una partícula), las distribuciones asociadas a partículas indistinguibles se diferencian en mayor medida de las asociadas a partículas distinguibles en el espacio de momento, en lo que concierne a su estructura.

En el nivel de dos partículas, la correlación entre las partículas debida a la indistinguibilidad es mayor en el espacio de momento, salvo en el estado basal, y en el caso de la función antisimétrica además para $n_{2}=3$; esto es importante porque la formulación más difundida de la mecánica cuántica es 
en el espacio de posición, y se muestra que hay información no despreciable en el espacio complementario. Como la información mutua puede ser interpretada como una diferencia entre la localización de la distribución en los niveles de una y dos partículas, se observa que en el espacio de momento existe un comportamiento que puede ser ligado con un fenómeno llamado condensación.

El comportamiento como función de a de la entropía de Shannon de las marginales de la densidad de pares y de la densidad de pares permite argumentar que si atendemos a la localización de la distribución, es más fácil distinguir entre partículas distinguibles e indistinguibles en el espacio de momentos, mientras que la distinción entre la distribución simétrica y antisimétrica es mayor en el espacio de posición.

El comportamiento constante con a para la suma entrópica se mantiene para las marginales de la densidad de pares y para la propia densidad de pares, lo cual es interesante porque implica que la información sobre el principio de incertidumbre está contenido en la misma forma que para unapartícula-en-una-caja, lo que es un indicativo de que la simetría no influye. Resalta el caso de la suma entrópica de la densidad de pares, porque es un principio de incertidumbre al nivel de dos partículas.

El punto de cruce $s_{\Psi}=s_{\widetilde{\Psi}}$ ocurre para valores menores de $a$ para partículas distinguibles que para indistinguibles, y entre estas últimas ocurre para la función simétrica en valores menores de a que para la función antisimétrica. De igual manera que en el nivel de una partícula, la distribución en espacio de posición está más localizada (condensación) que la de mo- 
106

mento en $a$ menores, y en $a$ mayores se invierte el comportamiento.

En lo que refiere a la posibilidad de diferenciar las partículas distinguibles de las indistinguibles atendiendo a la estructura de su distribución, es mayor esta diferencia en el espacio de momento, mientras que la diferencia de estructura entre las funciones simétrica y antisimétrica es mayor en el espacio de posición.

La correlación se mantiene constante en general como función de $a$.

Es posible escribir ecuaciones que permiten separar diferentes tipos de correlaciones en el nivel de dos partículas y relacionar fenómenos como la correlación proveniente de la indistinguibilidad y la correlación posiciónmomento, además del estudio de la influencia de la indistinguibilidad en fenómenos como el entanglement cuántico. 


\section{Perspectivas}

- Construir la función de Wigner para el modelo de dos-partículas-enuna-caja y estudiar la separación de los diferentes tipos de correlación.

- Incluir las correlaciones coulómbicas en el modelo de dos-partículasen-una-caja y analizar las relaciones entre los tres tipos de correlación que han sido mencionados.

- Analizar el efecto de la dimensionalidad en los modelos estudiados.

- Estudiar el efecto de la correlación posición-momento en las propiedades de sistemas atómicos y moleculares, y en el enlace químico. 


\section{Apéndice A}

\subsection{El enlace químico.}

El enlace químico covalente ha sido estudiado en una perspectiva energética utilizando las densidades de un electrón. Ha quedado establecida la relevancia de la indistinguibilidad, pues existen términos en la energía relacionados con el intercambio electrónico que son importantes para reproducir las curvas experimentales. A estos efectos se les asume como provenientes del formalismo cuántico, ya que no existe un análogo clásico.

Por otro lado, se ha argumentado que la deslocalización es un concepto clave en el mecanismo de formación del enlace químico $[55,56,57]$, lo cual vuelve interesante el cálculo de la entropía de Shannon para medirla.

En la perspectiva en que se ha argumentado en este trabajo, sería deseable un estudio de la distribución teniendo en cuenta su entropía de par electrónico y un estudio de la correlación posición-momento para analizar el efecto que ésta tiene en el enlace químico.

Existen en perspectiva dos aspectos para considerar. 
Primero, buscar una alternativa en el estudio del enlace químico covalente, analizando las densidades de pares electrónicos. La importancia de considerar densidades de pares es debida a que en química es frecuente considerar al enlace químico covalente como producto de compartir dos electrones, salvo en el caso de $H_{2}^{+}$.

Hemos comenzado el estudio de la molécula de hidrógeno utilizando las entropías de Shannon de la densidad de pares en el espacio de posición, obtenidas a partir de la función de Heitler y London [58], definida,

$$
\Psi=\frac{1}{\sqrt{2\left(1+S^{2}\right)}}\left[\phi_{A}(1) \phi_{B}(2)+\phi_{B}(1) \phi_{A}(2)\right]
$$

donde los subíndices $A$ y $B$ son las etiquetas para los núcleos y la dependencia (1) y (2) son las etiquetas sobre los electrones, y $S$ es el traslape de las funciones de onda. La función (9.1) puede escribirse utilizando orbitales tipo Slater y en coordenadas esferoidales [59],

$$
\begin{array}{r}
\Psi=\frac{1}{\sqrt{2\left(1+S^{2}\right)}}\left[\chi_{A}\left(\mu_{1}, \nu_{1}, \phi_{1}\right) \chi_{B}\left(\mu_{2}, \nu_{2}, \phi_{2}\right)\right. \\
\left.+\chi_{B}\left(\mu_{1}, \nu_{1}, \phi_{1}\right) \chi_{A}\left(\mu_{2}, \nu_{2}, \phi_{2}\right)\right] .
\end{array}
$$

En el estado basal debido a que no hay dependencia angular, la Ec. (9.2) es de cuatro variables y es más sencilla su manipulación numérica. Los intervalos de integración son, $\mu[1, \infty], \nu[-1,1]$ y $\phi[0,2 \pi]$, además,

$$
\chi_{B}(\mu, \nu, \phi)=\chi_{A}(\mu,-\nu, \phi)
$$


En este sistema de coordenadas, los orbitales tipo Slater para el estado basal, $n=1$, son,

$$
\chi_{A}(\mu, \nu, \phi)=\left(\frac{\xi^{3}}{\pi}\right)^{\frac{1}{2}} e^{\frac{-\xi R \mu}{2}} e^{\frac{-\xi R \nu}{2}}
$$

donde $R$ es la distancia internuclear y $\xi$ es un parámetro variacional, en este trabajo fue impuesto $\xi=1$ (como en el cálculo original de Heitler y London). Para la integración, no debe olvidarse el elemento de volumen,

$$
\frac{R^{3}}{8}\left(\mu^{2}-\nu^{2}\right) d \mu d \nu d \phi
$$

La integración numérica en cuatro dimensiones se realizó con una cuadratura de Gauss-Legendre de 12 puntos en cada subintervalo, con precisión doble.

Las figuras (9.1) y (9.2) muestran las entropías de uno y dos electrones, respectivamente. En este caso, en la entropía monoelectrónica no se observa estructura que pueda relacionarse con la formación del enlace químico, por otra parte, de la entropía de pares electrónicos puede argumentarse que en la curva se observa la formación del enlace químico, por el máximo que presenta.

El análisis de la figura (9.2) puede hacerse como sigue: si nos movemos de cero a distancias internucleares más grandes, observamos primero una deslocalización de la distribución de pares, como consecuencia de aumentar el espacio disponible para el movimiento electrónico, pero después de $R=$ 3.2 (donde ocurre el máximo $\left.s_{2 p a r t}=9.0865\right)$, se observa una localización en la distribución, la interpretación física es que cada electrón comienza a 


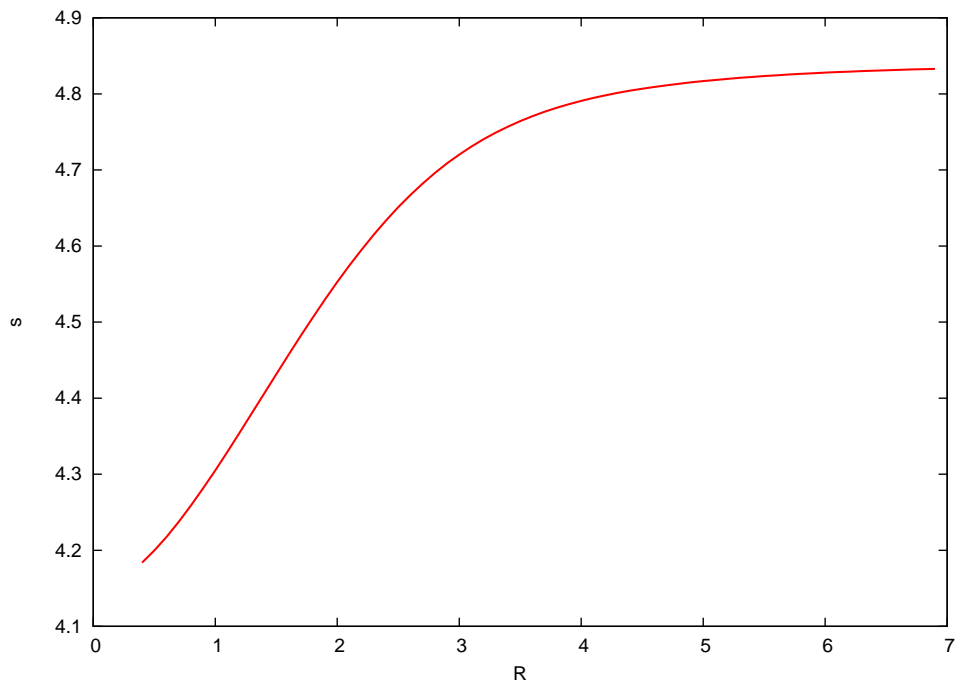

Figura 9.1: Entropía monoelectrónica usando la función de Heitler y London como función de la distancia internuclear $R$. 


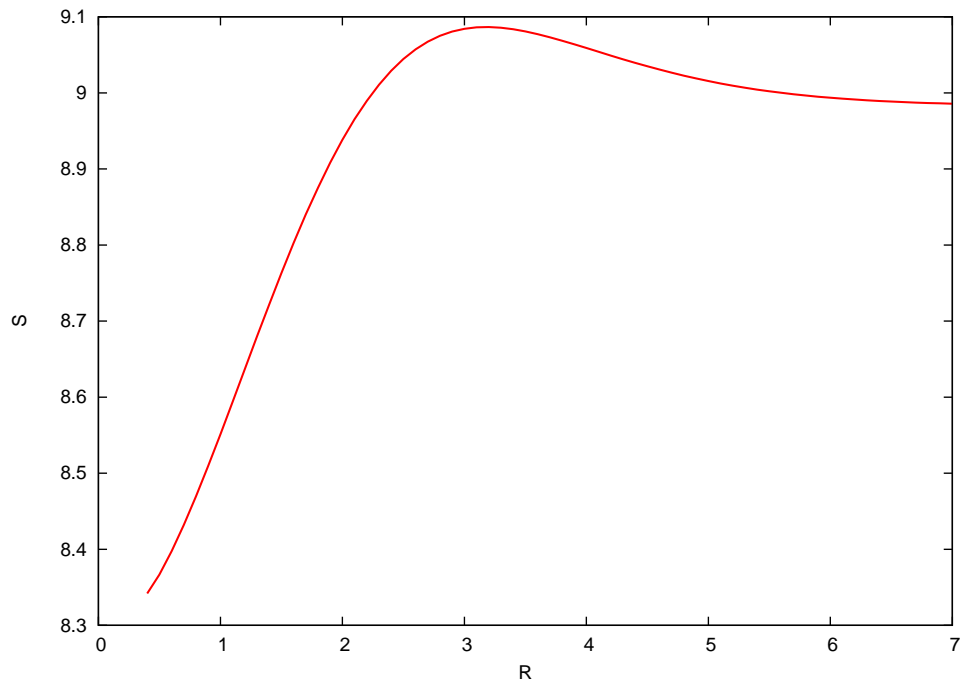

Figura 9.2: Entropía bielectrónica usando la función de Heitler y London como función de la distancia internuclear $R$. 
localizarse alrededor de cada núcleo. Se encuentra en perspectiva extender este análisis al espacio de momento.

Segundo, también se encuentra en perspectiva considerar la formulación en espacio-fase de sistemas atómicos [60] y moleculares para estudiar la influencia de la correlación posición-momento en la formación del enlace químico covalente y, más general, su influencia en las propiedades de los sistemas atómicos y moleculares. 


\section{Apéndice B}

\subsection{Una-partícula-en-una-caja: Tablas.}

Tabla 1. Entropía de Shannon de la función de Wigner y suma entrópica.

\begin{tabular}{|c|c|c|c|c|}
\hline$n$ & $\operatorname{Re}\left[s_{w}\right]$ & $\operatorname{Im}\left[s_{w}\right]$ & $\left|s_{w}\right|$ & $s_{\psi}+s_{\widetilde{\psi}}$ \\
\hline 1 & 2.0983 & 0.1862 & 2.1065 & 2.2120 \\
2 & 2.6224 & 0.9368 & 2.7847 & 2.6070 \\
3 & 2.6799 & 1.3739 & 3.0115 & 2.7531 \\
4 & 2.7738 & 1.6820 & 3.2440 & 2.8292 \\
5 & 2.7920 & 1.9199 & 3.3884 & 2.8758 \\
6 & 2.8303 & 2.1135 & 3.5324 & 2.9074 \\
7 & 2.8391 & 2.2768 & 3.6392 & 2.9308 \\
8 & 2.8598 & 2.4179 & 3.7450 & 2.9477 \\
9 & 2.8650 & 2.5421 & 3.8302 & 2.9602 \\
10 & 2.8780 & 2.6529 & 3.9142 & 2.9717 \\
\hline
\end{tabular}


116

Tabla 2. Información mutua. Correlación posición-momento.

\begin{tabular}{|c|c|c|c|c|}
\hline$n$ & $\operatorname{Re}\left[I_{w}\right]$ & $\operatorname{Im}\left[I_{w}\right]$ & $\left|I_{w}\right|$ & $I_{w_{(\text {sum_normas })}}$ \\
\hline 1 & 0.1137 & -0.1862 & 0.2182 & 0.1055 \\
2 & -0.0153 & -0.9368 & 0.9370 & -0.1776 \\
3 & 0.0733 & -1.3739 & 1.3758 & -0.2584 \\
4 & 0.0554 & -1.6820 & 1.6829 & -0.4148 \\
5 & 0.0838 & -1.9199 & 1.9217 & -0.5126 \\
6 & 0.0771 & -2.1135 & 2.1149 & -0.6249 \\
7 & 0.0917 & -2.2768 & 2.2786 & -0.7085 \\
8 & 0.0879 & -2.4179 & 2.4195 & -0.7972 \\
9 & 0.0952 & -2.5421 & 2.5439 & -0.8700 \\
10 & 0.0938 & -2.6529 & 2.6546 & -0.9425 \\
\hline
\end{tabular}

La entropía de Shannon de la función de Wigner y la suma entrópica son independientes de $a$, el tamaño de la caja, por lo tanto también lo es la información mutua. 


\subsection{Dos-partículas-en una-caja: Tablas.}

Tabla 3. Entropía de las marginales de la densidad de pares para los estados $\left(n_{1}=1, n_{2}\right)$.

\begin{tabular}{|c|c|c|c|c|c|c|}
\hline$n_{2}$ & $s_{\psi_{I}}$ & $s_{\widetilde{\psi}_{I}}$ & $s_{\psi_{D}}$ & $s_{\widetilde{\psi}_{D}}$ & $s_{\psi_{I}}+s_{\widetilde{\psi}_{I}}$ & $s_{\psi_{D}}+s_{\widetilde{\psi}_{D}}$ \\
\hline 1 & 0.0000 & 0.0000 & -0.3069 & 2.5189 & 0.0000 & 2.2120 \\
2 & -0.1716 & 2.9698 & -0.3069 & 2.5189 & 2.7982 & 2.2120 \\
3 & -0.1509 & 3.2719 & -0.3069 & 2.5189 & 3.1210 & 2.2120 \\
4 & -0.1460 & 3.3934 & -0.3069 & 2.5189 & 3.2475 & 2.2120 \\
5 & -0.1444 & 3.4466 & -0.3069 & 2.5189 & 3.3022 & 2.2120 \\
6 & -0.1439 & 3.4932 & -0.3069 & 2.5189 & 3.3493 & 2.2120 \\
7 & -0.1436 & 3.5120 & -0.3069 & 2.5189 & 3.3684 & 2.2120 \\
8 & -0.1435 & 3.5372 & -0.3069 & 2.5189 & 3.3937 & 2.2120 \\
9 & -0.1434 & 3.5456 & -0.3069 & 2.5189 & 3.4021 & 2.2120 \\
10 & -0.1434 & 3.5616 & -0.3069 & 2.5189 & 3.4182 & 2.2120 \\
\hline
\end{tabular}

Los subíndices $I$ y $D$ corresponden a Indinstiguibles y Distinguibles, respectivamente. 
Tabla 4. Entropía de pares para los estados $\left(n_{1}=1, n_{2}\right)$.

\begin{tabular}{|c|c|c|c|c|c|c|}
\hline$n_{2}$ & $s_{\boldsymbol{\Psi}_{\mathbf{A}}}$ & $s_{\widetilde{\boldsymbol{\Psi}}_{\mathbf{A}}}$ & $s_{\boldsymbol{\Psi}_{\mathbf{S}}}$ & $s_{\widetilde{\boldsymbol{\Psi}}_{\mathbf{S}}}$ & $s_{\boldsymbol{\Psi}_{\mathbf{N}}}$ & $s_{\widetilde{\Psi}_{\mathbf{N}}}$ \\
\hline 1 & 0.0000 & 0.0000 & -0.6137 & 5.0378 & -0.6137 & 5.0378 \\
2 & -0.7539 & 5.6239 & -0.7539 & 5.6239 & -0.6137 & 5.4327 \\
3 & -0.7539 & 6.1041 & -0.6066 & 6.1780 & -0.6137 & 5.5789 \\
4 & -0.6694 & 6.2811 & -0.6694 & 6.2811 & -0.6137 & 5.6549 \\
5 & -0.6794 & 6.3430 & -0.6545 & 6.3686 & -0.6137 & 5.7015 \\
6 & -0.6661 & 6.3998 & -0.6661 & 6.3998 & -0.6137 & 5.7330 \\
7 & -0.6696 & 6.4237 & -0.6618 & 6.4369 & -0.6137 & 5.7557 \\
8 & -0.6655 & 6.4518 & -0.6655 & 6.4518 & -0.6137 & 5.7728 \\
9 & -0.6670 & 6.4644 & -0.6638 & 6.4725 & -0.6137 & 5.7862 \\
10 & -0.6653 & 6.4813 & -0.6653 & 6.4813 & -0.6137 & 5.7970 \\
\hline
\end{tabular}


Tabla 5. Suma de la entropía de pares para los estados $\left(n_{1}=1, n_{2}\right)$.

\begin{tabular}{|c|c|c|c|}
\hline$n_{2}$ & $s_{\boldsymbol{\Psi}_{\mathbf{A}}}+s_{\widetilde{\boldsymbol{\Psi}}_{\mathbf{A}}}$ & $s_{\boldsymbol{\Psi}_{\mathbf{S}}}+s_{\widetilde{\boldsymbol{\Psi}}_{\mathbf{S}}}$ & $s_{\boldsymbol{\Psi}_{\mathbf{N}}}+s_{\widetilde{\boldsymbol{\Psi}}_{\mathbf{N}}}$ \\
\hline 1 & 0.0000 & 4.4241 & 4.4241 \\
2 & 4.8700 & 4.8700 & 4.8190 \\
3 & 5.3502 & 5.5714 & 4.9652 \\
4 & 5.6118 & 5.6118 & 5.0412 \\
5 & 5.6636 & 5.7141 & 5.0878 \\
6 & 5.7338 & 5.7338 & 5.1192 \\
7 & 5.7541 & 5.7751 & 5.1420 \\
8 & 5.7863 & 5.7863 & 5.1589 \\
9 & 5.7974 & 5.8088 & 5.1724 \\
10 & 5.8159 & 5.8159 & 5.1830 \\
\hline
\end{tabular}


Tabla 6. Información mutua. Correlación proveniente de la indistinguibilidad para los estados $\left(n_{1}=1, n_{2}\right)$.

\begin{tabular}{|c|c|c|c|c|}
\hline$n_{2}$ & $I_{x, A}$ & $I_{p, A}$ & $I_{x, S}$ & $I_{p, S}$ \\
\hline 1 & 0.0000 & 0.0000 & 0.0000 & 0.0000 \\
2 & 0.4107 & 0.3158 & 0.4107 & 0.3158 \\
3 & 0.4521 & 0.4397 & 0.3048 & 0.3658 \\
4 & 0.3775 & 0.5057 & 0.3775 & 0.5057 \\
5 & 0.3906 & 0.5503 & 0.3656 & 0.5247 \\
6 & 0.3783 & 0.5865 & 0.3783 & 0.5865 \\
7 & 0.3823 & 0.6003 & 0.3745 & 0.5871 \\
8 & 0.3785 & 0.6225 & 0.3785 & 0.6225 \\
9 & 0.3801 & 0.6267 & 0.3769 & 0.6186 \\
10 & 0.3785 & 0.6420 & 0.3785 & 0.6420 \\
\hline
\end{tabular}

Para la entropía de las marginales, la de pares y la información mutua, los subíndices utilizados fueron: $A$ para funciones Antisimétricas, $S$ para Simétricas y $N$ para No simétricas. 


\section{Bibliografía}

[1] W. Kutzelnigg, G. Del Re, G. Berthier, Phys. Rev., 172, 49 (1968).

[2] R. P. Sagar, N. L. Guevara, J. Chem. Phys., 123, 044108 (2005).

[3] R. P. Sagar, N. L. Guevara, J. Chem. Phys., 124, 134101 (2006).

[4] A. Anderson, Phys. Rev. D, 42, 585 (1990).

[5] J. J. Halliwell, Phys. Rev. D, 36, 3626 (1987).

[6] C. E. Shannon, Bell Syst. Tech. J. 27, 379 (1948), reimpreso en Claude Elwood Shannon: collected papers, C. E. Shannon, IEEE Press, New York (1993).

[7] R. P. Sagar, N. L. Guevara, J. Chem. Phys., 123, 044108 (2005).

[8] V. Majerník y Richterek, J. Phys. A: Math. Gen., 30, L49 (1997).

[9] V. Majerník, R. Charvot y E. Majerníková J. Phys. A: Math. Gen. 32, 2207 (1999).

[10] I. Novak, J. Chem. Educ., 78, 395 (2001). 
[11] E. P. Wigner, Phys. Rev., 40, 749 (1932).

[12] L. de la Peña, Introducción a la Mecánica Cuántica, Universidad Nacional Autónoma de México - Fondo de Cultura Económica (2006).

[13] W. Heisenberg, Z. für Physik 43, 172 (1927). Traducción al inglés en J. A. Wheeler y H. Zurek, Quantum Theory and Measurement, Princeton Univ. Press (1983).

[14] E. Schrödinger, Proceedings of the Prussian Academy of Sciences, Physics-Mathematical section, XIX, 296 (1930). Publicado en inglés en arXiv:quant-ph/9903100v2 (2000).

[15] I. Bialynicki-Birula y J. Mycielski, Commun. Math. Phys., 44, 129 (1975).

[16] N. L. Guevara, R. P. Sagar, R. O. Esquivel, J. Chem. Phys. 119, 7030 (2003).

[17] U. Leonhardt, Measuring the Quantum State of Light, Cambridge University Press, U.S.A. (1970).

[18] M. Hillery, R. F. O'connell, M. O. Scully, E. P. Wigner, Phys. Rep., 106, 121 (1984).

[19] V. I. Tatarskii, Usp. Fiz. Nauk., 139, 587 (1983). Traducción al inglés en, Sov. Phys. Usp., 26, 4 (1983). 
[20] M. A. Nielsen, I. L. Chuang, Quantum Computation and Quantum Information, Cambridge University (2000).

[21] R. G. Parr, L. J. Bartolotti, J. Phys. Chem., 87, 2810 (1983).

[22] T. Cover y J. Thomas, Elements of Information Theory, John Wiley and Sons (1991).

[23] S. Kullback, Information Theory and Statistics, Dover Pub., Gloucester (1978).

[24] D. Bohm, Quantum Theory, Dover, New York (1989).

[25] A. Julg, Theor. Chim. Acta, 74, 323 (1988).

[26] A. Wehrl, Rev. Mod. Phys., 50, 221 (1978).

[27] K. Piatek, W. Leoński, J. Phys. A: Math. Gen., 34, 4951 (2001).

[28] J. J. Wlodarz, Int. J. Theor. Phys., 42, 1075 (2003).

[29] G. Manfredi, M. R. Feix, Phys. Rev. E, 62, 4665 (2000).

[30] S. Aviyente, W. J. Williams, Proc. IEEE International Conference on Acoustics, 6, VI-441 (2003).

[31] E. H. Allen, Philosophy of Science, 43, 53 (1976).

[32] Ch. Kurtsiefer; T. Pfau; J. Mlynek, Nature, 386, 150 (1997).

[33] G. Nogues, et. al., Phys. Rev. A, 62, 054101 (2000). 
[34] J. P. Dahl, Physica, 114A, 439 (1982).

[35] A. N. Kolmogorov, Foundations of the Theory of Probability, Chelsea Pub. Co., New York (1956).

[36] W. Mückenheim, Phys. Rep., 133, 337 (1986).

[37] R. Feynman, en B. Hiley, F. Peat, eds., Essays in Honor of David Bohm, Routledge and Kegan Paul, London, 235 (1987), reimpreso en D. B. Fairlie, T. L. Curtright, Quantum Mechanics in Phase Space: An Overview with Selected Papers, World Scientific, New Jersey, 426 (2005).

[38] J. B. Hartle, Phys. Rev. A, 78, 012108 (2008).

[39] S. N. Gupta, Quantum Electrodynamics, Gordon and Breach, London (1977).

[40] J. P. Dahl, H. Mack, A. Wolf, W. P. Schleich, Phys. Rev. A, 74, 042323 (2006).

[41] J. Sperling, W. Vogel, arXiv:quant-ph/0811.4527v1 (2008).

[42] A. C. de la Torre, J. L. Iguain, Eur. J. Phys., 19, 563 (1998).

[43] G. M. Shalhoub, J. Chem. Educ., 74, 1317 (1997).

[44] W. D. Hobey, J. Org. Chem., 37, 1137 (1972). 
[45] D. Hoekzema, E. van den Berg, G. Schooten, L. van Dijk, Physics Education, 42, 391 (2007).

[46] T. G. Pedersen, Phys. Rev. B, 62, 15424 (2006).

[47] M. H. Weber, K. G. Lynn, B. Barbiellini, P. A. Sterne, A. B. Denison, Phys. Rev. B, 66, 041305 (2002).

[48] F. Rioux, J. Chem. Educ., 76, 156 (1999).

[49] J. Peslak, Am. J. Phys., 47, 39 (1979).

[50] J. Sánchez-Ruiz, Phys. Lett. A, 226, 7 (1997).

[51] M. Belloni, M. A. Doncheski, R. W. Robinett, Am. J. Phys., 72, 1183 (2004).

[52] J. M. Lévy-Leblond, Am. J. Phys., 54, 135 (1986).

[53] Wolfram Research, Inc., Mathematica, Version 6.0, Champaign, IL, (2006).

[54] A. Einstein, B. Podolsky, N. Rosen, Phys. Rev., 47, 777 (1935).

[55] K. Ruedenberg, Rev. Mod. Phys., 34, 326 (1962).

[56] W. Kutzelnigg, Angew. Chem. Int. Ed., 12, 546 (1973).

[57] S. Nordholm, J. Chem. Educ., 65, 581 (1988).

[58] W. Heitler, F. London, Z. Physik, 44, 455 (1927). 
[59] L. J. Schaad, I. L. Thomas, Theoret. Chim. Acta, 21, 115 (1971).

[60] L. Praxmeyer, J. Mostowski, K. Wódkiewicz, J. Phys. A, 39, 14143 (2006) 